\title{
Benchmarking of the Mono-Energetic Transport Coefficients - Results From the International Collaboration on Neoclassical Transport in Stellarators (ICNTS)
}

\author{
C.D. Beidler ${ }^{1}$, K. Allmaier ${ }^{2}$, M.Yu. Isaev ${ }^{3}$, S.V. Kasilov ${ }^{2,4}$, W. Kernbichler ${ }^{2}$, \\ G.O. Leitold ${ }^{2}$, H. Maaßberg ${ }^{1}$, D.R. Mikkelsen ${ }^{5}$, S. Murakami ${ }^{6}$, \\ M. Schmidt ${ }^{1}$, D.A. Spong ${ }^{7}$, V. Tribaldos ${ }^{8}$, A. Wakasa ${ }^{6}$ \\ ${ }^{1}$ Max-Planck-Institut für Plasmaphysik, IPP-EURATOM Association, Greifswald, GERMANY \\ ${ }^{2}$ Technische Universität Graz, ÖAW-EURATOM Association, Graz, AUSTRIA \\ ${ }^{3}$ Nuclear Fusion Institute, Russian Research Centre "Kurchatov Institute”, Moscow, RUSSIA \\ ${ }^{4}$ Institute of Plasma Physics, NSC "Kharkov Institute of Physics and Technology", Kharkov, UKRAINE \\ ${ }^{5}$ Princeton Plasma Physics Laboratory, Princeton NJ, USA \\ ${ }^{6}$ Department of Nuclear Engineering, Kyoto University, Kyoto, JAPAN \\ ${ }^{7}$ Oak Ridge National Laboratory, Oak Ridge TN, USA \\ ${ }^{8}$ Departamento de Física, Universidad Carlos III de Madrid, Leganés, SPAIN
}

E-mail: Craig.Beidler@ipp.mpg.de

\begin{abstract}
Numerical results for the three mono-energetic transport coefficients required for a complete neoclassical description of stellarator plasmas have been benchmarked within an international collaboration. These transport coefficients are flux-surface-averaged moments of solutions to the linearised drift kinetic equation which have been determined using field-line-integration techniques, Monte Carlo simulations, a variational method employing Fourier-Legendre test functions and a finite difference scheme. The benchmarking has been successfully carried out for past, present and future devices which represent different optimisation strategies within the extensive configuration space available to stellarators. A qualitative comparison of the results with theoretical expectations for simple model fields is provided. The behaviour of the results for the mono-energetic radial and parallel transport coefficients can be largely understood from such theoretical considerations but the mono-energetic bootstrap current coefficient exhibits characteristics which have not been predicted.
\end{abstract}




\section{Introduction}

This special topics paper describes work carried out within the IEA Implementing Agreement for Cooperation in Development of the Stellarator Concept, with the ultimate goal of providing a comprehensive description of neoclassical transport processes in stellarator experiments. As in the case of axisymmetric tokamaks such a description is essential for calculating the expected flows within a flux surface (e.g. determining the bootstrap current and the parallel electric conductivity) but, additionally, the neoclassical transport across flux surfaces represents a considerable limitation on plasma confinement in stellarators due to its strong temperature dependence (for example, in the most unfavourable case the radial energy flux scales as $T^{9 / 2}$ in stellarators in contrast with the far more benign $T^{1 / 2}$ scaling which holds in the tokamak banana regime or the $T^{5 / 2}$ dependence expected from gyro-Bohm turbulent transport). This has obvious implications for stellarator reactor prospects but can also be of relevance for experiments of moderate size as demonstrated by various high-performance discharges in the W7-AS device which conformed with neoclassical expectations for both particle and energy confinement [1-3]. An additional prediction of stellarator neoclassical theory concerning radial transport - the possibility of multiple solutions for the value of radial electric field required to satisfy the ambipolarity constraint on electron and ion particle fluxes - has also been confirmed by experimental observations on the LHD, CHS, TJ-II and W7-AS devices in general accordance with theoretical expectations $[4,5]$.

Neoclassical theory for toroidal devices is commonly considered to be a mature field of research given the extensive body of scientific literature dealing with the topic; for stellarators the two most comprehensive treatments of the subject matter are presented in review articles $[6,7]$. Practical use of such theoretical results is circumscribed, however, due to the rather simple magnetic fields which are assumed for analytic calculations of the geometrical factors relevant to neoclassical transport. Unfortunately, these geometrical factors are often quite sensitive to details of the magnetic field structure, especially in the case of strong three-dimensional (3-D) shaping of the stellarator's magnetic flux surfaces regardless of whether such shaping is intrinsic to the magnetic configuration or attributable to a finite-pressure equilibrium (or a combination of the two). An additional practical drawback of the analytic results is the asymptotic nature of their validity, being appropriate for a particular set of ordering assumptions which may be only approximately fulfilled under realistic experimental conditions.

Computational methods for determining neoclassical transport in stellarators have been developed to avoid (or at least ameliorate) such shortcomings of the analytic theory and their number has become appreciable over the past years. Historically, the results obtained with such numerical tools have been presented in a variety of ways depending on the desired application, although for determining neoclassical contributions to the transport of plasma observables such as density, temperature and current it is most convenient to employ the three so-called mono- 
energetic transport coefficients. These flux-surface-averaged moments of the solution to the linearised drift kinetic equation are particularly attractive as they contain sufficient information to calculate all neoclassical fluxes/flows for (nearly) arbitrary plasma parameters in a given magnetic field configuration. At the initiation of the International Collaboration on Neoclassical Transport in Stellarators (ICNTS), it was therefore a natural first order of business to carry out and document a thorough benchmarking of various numerical methods used within the stellarator community to calculate the mono-energetic transport coefficients for realistic 3-D magnetic-field configurations. In this manner each computational tool has been exposed to far more comprehensive investigations than in previous comparisons of code results which have typically involved the application of two numerical approaches to a single device. The results of this benchmarking activity, described in the following, were obtained using the field-lineintegration techniques of the NEO family of codes $[8,9]$, Monte Carlo simulations employing either full- $f$ [10-12] or $\delta f$ schemes [13-15], the variational approach of the Drift Kinetic Equation Solver, DKES $[16,17]$ and (where appropriate) a numerical solution of the rippleaveraged kinetic equation, GSRAKE [18]. The devices for which the benchmarking has been performed are representative of the extensive configuration space available to stellarators: the Compact Helical System (CHS) and Large Helical Device (LHD) heliotrons, both located at Toki, Japan; the Helically-Symmetric Experiment (HSX), in operation at Madison, WI, USA; the quasi-axisymmetric National Compact Stellarator Experiment (NCSX), under construction until recently at Princeton, NJ, USA; the Quasi-Poloidal Stellarator (QPS), a design study initiated by Oak Ridge National Laboratory, USA; the heliac TJ-II, in operation at Madrid, Spain; an example of a Quasi-Isodynamic stellarator with Poloidally Closed contours of the magnetic field strength (QIPC), taken from the literature; and two advanced stellarators of the Wendelstein line, W7-AS which ended operation in 2002 at Garching, Germany, and the helias W7-X which is under construction at Greifswald, Germany.

The fundamentals of neoclassical transport theory in stellarators are outlined in Section 2 of this paper, beginning with the linearised drift kinetic equation used to describe the plasma at the microscopic level and culminating in the definition of the mono-energetic transport coefficients which enable efficient use of the kinetic equation's solution in the macroscopic transport equations. Section 3 provides a brief overview of the methods used to solve this kinetic equation including consideration of each method's strengths and weaknesses. Section 4 presents the magnetic configurations for which transport coefficients have been determined and a discussion of the neoclassical optimisation strategy which each device follows. A sample of benchmarking results is then given in Section 5 and the paper concludes with a number of observations and remarks. 


\section{Basics}

Neoclassical theory describes transport processes which are assumed to be radially local and described by the linearised drift kinetic equation [16]

$$
\mathcal{V}\left(f_{1}\right)-\nu \mathcal{L}\left(f_{1}\right)=-\frac{\mathrm{d} r}{\mathrm{~d} t}\left(\frac{1}{n} \frac{\mathrm{d} n}{\mathrm{~d} r}-\frac{q E_{r}}{T}+\left(K-\frac{3}{2}\right) \frac{1}{T} \frac{\mathrm{d} T}{\mathrm{~d} r}\right) f_{M}+p v B \frac{q\langle\mathbf{E} \cdot \mathbf{B}\rangle}{T\left\langle B^{2}\right\rangle} f_{M}
$$

where $f_{1}$ is the (small) deviation of the distribution function from Maxwellian and

$$
\mathcal{V}\left(f_{1}\right)=\left(p v \frac{\mathbf{B}}{B}+\frac{\mathbf{E}_{r} \times \mathbf{B}}{\left\langle B^{2}\right\rangle}\right) \cdot \nabla f_{1}-\frac{v\left(1-p^{2}\right)}{2 B^{2}} \mathbf{B} \cdot \boldsymbol{\nabla} B \frac{\partial f_{1}}{\partial p}
$$

is the Vlasov operator, with $\mathbf{B}$ the magnetic-field vector, $B$ its magnitude, $v$ is the particle speed, $p=v_{\|} / v$ the pitch-angle variable, $\mathbf{E}_{r}=E_{r} \boldsymbol{\nabla} r$ is the radial electric field, $r$ is the flux-surface label, angle brackets denote the flux-surface average and $\nu \mathcal{L}$ is the Lorentz pitchangle-scattering collision operator with

$$
\mathcal{L}\left(f_{1}\right)=\frac{1}{2} \frac{\partial}{\partial p}\left(\left(1-p^{2}\right) \frac{\partial f_{1}}{\partial p}\right) .
$$

The radial drift velocity is given by

$$
\frac{\mathrm{d} r}{\mathrm{~d} t}=\frac{m v^{2}\left(1+p^{2}\right)}{2 q B^{3}}(\mathbf{B} \times \nabla B) \cdot \nabla r
$$

where $m$ is the particle mass and $q$ its charge, $n$ and $T$ are the density and temperature, respectively, of the local Maxwellian $f_{M}=n(m / 2 \pi T)^{3 / 2} \exp (-K), K=m v^{2} / 2 T$ is the normalised kinetic energy and the term containing $\langle\mathbf{E} \cdot \mathbf{B}\rangle$ describes the effects of the parallel electric field which appears in response to an externally applied loop voltage. An explicit particle-species index has not been attached to any of the quantities appearing in eq. (1), it being understood that all such quantities are those of the species of interest (otherwise assigned the index $\alpha$ ). Only in a single case is it necessary to abandon this convention as the collision frequency for particle species $\alpha$

$$
\nu \equiv \nu^{\alpha}=\sum_{\beta} \nu^{\alpha / \beta}
$$

is given by the sum of "discrete" collision frequencies with each of the background plasma species $\beta$ (including like-particle collisions)

$$
\nu^{\alpha / \beta}=\nu_{0}^{\alpha / \beta}\left\{\operatorname{erf}\left(\sqrt{\mathrm{K}^{\alpha / \beta}}\right)\left(1-\frac{1}{2 \mathrm{~K}^{\alpha / \beta}}\right)+\left(\pi \mathrm{K}^{\alpha / \beta}\right)^{-1 / 2} \exp \left(-\mathrm{K}^{\alpha / \beta}\right)\right\},
$$

in which the reference collision frequency is

$$
\nu_{0}^{\alpha / \beta}=\frac{n^{\beta}\left(q^{\alpha} q^{\beta}\right)^{2}(\ln \Lambda)^{\alpha / \beta}}{4 \pi\left(\varepsilon_{0} m^{\alpha}\right)^{2} v^{3}},
$$


$K^{\alpha / \beta}=\left(m^{\beta} / m^{\alpha}\right)\left(T^{\alpha} / T^{\beta}\right) K, \ln \Lambda$ is the Coulomb logarithm, $\varepsilon_{0}$ is the permittivity of free space, and the error function is defined in the usual manner

$$
\operatorname{erf}(\mathrm{y})=\frac{2}{\sqrt{\pi}} \int_{0}^{\mathrm{y}} \mathrm{d} \xi \exp \left(-\xi^{2}\right) .
$$

In the literature, $\nu$ is commonly referred to as the deflection collision frequency and the notation $\nu_{d}$ is used (or $\nu_{\perp} / 2$ ) to distinguish it from frequencies which characterise other collisional processes. In the current paper, it is the only collision frequency of relevance and the subscript has therefore been dropped.

It will be noted that derivatives of $f_{1}$ with respect to $r$ and $v$ are lacking in eq. (1) making it possible to treat these two variables as mere parameters. This represents a considerable simplification of the general drift kinetic equation from five phase-space variables to a more manageable three. It also allows one to streamline the notation by expressing the first-order distribution function

$$
f_{1}=-\frac{q R_{0} B_{0}\langle\mathbf{E} \cdot \mathbf{B}\rangle}{T\left\langle B^{2}\right\rangle} f_{M} \widehat{f}_{I}+\frac{v_{d} R_{0}}{v}\left(\frac{1}{n} \frac{\mathrm{d} n}{\mathrm{~d} r}-\frac{q E_{r}}{T}+\left(K-\frac{3}{2}\right) \frac{1}{T} \frac{\mathrm{d} T}{\mathrm{~d} r}\right) f_{M} \widehat{f}_{I I}
$$

where $R_{0}$ and $B_{0}$ are reference values of the torus major radius and magnetic field strength, respectively, and $v_{d}=m v^{2} /\left(2 q R_{0} B_{0}\right)$ is characteristic of the radial drift velocity. Written in dimensionless form, the resulting differential equations for $\widehat{f}_{I}$ and $\widehat{f}_{I I}$ (which are themselves dimensionless) are expressed

$$
\begin{gathered}
\frac{R_{0}}{v} \mathcal{V}\left(\widehat{f}_{I}\right)-\frac{R_{0} \nu}{v} \mathcal{L}\left(\widehat{f}_{I}\right)=-p \frac{B}{B_{0}} \\
\frac{R_{0}}{v} \mathcal{V}\left(\widehat{f}_{I I}\right)-\frac{R_{0} \nu}{v} \mathcal{L}\left(\widehat{f}_{I I}\right)=-\frac{1}{v_{d}} \frac{\mathrm{d} r}{\mathrm{~d} t} .
\end{gathered}
$$

In the literature, these two equations are often said to govern the transport in the parallel and radial directions, respectively. On examination, one quickly concludes that their solutions can depend only on the normalised $\mathbf{E}_{r} \times \mathbf{B}$ drift velocity, $v_{E}^{\star}=E_{r} /\left(v B_{0}\right)$, the normalised "mean-free-path", $\lambda^{\star}=v /\left(R_{0} \nu\right)$, and the structure of the confining magnetic field (but not its magnitude). Perhaps surprisingly, the solutions are thus formally independent of the particle species, although relevant values of $v_{E}^{\star}$ for electrons will typically be much smaller than those appropriate for ions.

Within this neoclassical formalism, the relationships between the flux-surface-averaged flows, $I_{i}$, and the thermodynamic forces which drive them, $A_{j}$, may then be expressed

$$
I_{i}=-n \sum_{j=1}^{3} L_{i j} A_{j}
$$


Conforming to the standard convention, $I_{1}$ is related to the radial component of the particle flux density, $\Gamma$, through

$$
I_{1}=\langle\boldsymbol{\Gamma} \cdot \boldsymbol{\nabla} r\rangle=\left\langle\int \mathrm{d}^{3} v \frac{\mathrm{d} r}{\mathrm{~d} t} f_{1}\right\rangle,
$$

$I_{2}$ to the radial component of the energy flux density, $\mathrm{Q}$,

$$
I_{2}=\left\langle\frac{\mathbf{Q}}{T} \cdot \nabla r\right\rangle=\left\langle\int \mathrm{d}^{3} v K \frac{\mathrm{d} r}{\mathrm{~d} t} f_{1}\right\rangle
$$

and $I_{3}$ to the parallel component of the current density, $\mathbf{J}$,

$$
I_{3}=\frac{\langle\mathbf{J} \cdot \mathbf{B}\rangle}{q B_{0}}=\left\langle\int \mathrm{d}^{3} v p v \frac{B}{B_{0}} f_{1}\right\rangle .
$$

By choosing to combine the thermodynamic forces in the following manner

$$
A_{1}=\frac{1}{n} \frac{\mathrm{d} n}{\mathrm{~d} r}-\frac{q E_{r}}{T}-\frac{3}{2} \frac{1}{T} \frac{\mathrm{d} T}{\mathrm{~d} r} \quad A_{2}=\frac{1}{T} \frac{\mathrm{d} T}{\mathrm{~d} r} \quad A_{3}=-\frac{q B_{0}\langle\mathbf{E} \cdot \mathbf{B}\rangle}{T\left\langle B^{2}\right\rangle}
$$

the mono-energetic solutions of the kinetic equations (2) and (3) may be used to determine the transport coefficients by energy convolution with the local Maxwellian

$$
L_{i j}=\frac{2}{\sqrt{\pi}} \int_{0}^{\infty} \mathrm{d} K \sqrt{K} e^{-K} D_{i j}(K) h_{i} h_{j}
$$

where $h_{1}=h_{3}=1, h_{2}=K$ and the $D_{i j}$ are mono-energetic transport coefficients defined by

$$
\begin{gathered}
D_{11}=D_{12}=D_{21}=D_{22}=-\frac{v_{d}^{2} R_{0}}{2 v}\left\langle\int_{-1}^{1} \mathrm{~d} p \frac{1}{v_{d}} \frac{\mathrm{d} r}{\mathrm{~d} t} \widehat{f}_{I I}\right\rangle \\
D_{13}=D_{23}=-\frac{v_{d} R_{0}}{2}\left\langle\int_{-1}^{1} \mathrm{~d} p \frac{1}{v_{d}} \frac{\mathrm{d} r}{\mathrm{~d} t} \widehat{f}_{I}\right\rangle \\
D_{31}=D_{32}=-\frac{v_{d} R_{0}}{2}\left\langle\int_{-1}^{1} \mathrm{~d} p p \frac{B}{B_{0}} \widehat{f}_{I I}\right\rangle \\
D_{33}=-\frac{v R_{0}}{2}\left\langle\int_{-1}^{1} \mathrm{~d} p p \frac{B}{B_{0}} \widehat{f}_{I}\right\rangle .
\end{gathered}
$$

Of these mono-energetic coefficients, $D_{11}$ is said to describe the radial transport, $D_{33}$ the parallel transport, $D_{13}$ is characteristic of the Ware pinch and $D_{31}$ of the bootstrap current. Only three of these coefficients are independent, however, as $D_{13}=-D_{31}$ due to Onsager symmetry.

Having knowledge of the radial profiles of these mono-energetic coefficients for relevant values of $v_{E}^{\star}$ and $\lambda^{\star}$ allows rapid determination of the neoclassical contributions to the macroscopic fluxes/flows which appear in the 1-D transport equations for plasma observables such as density, temperature and current. It is especially worth noting in this context, that the neoclassical fluxes/flows obtained by a straightforward application of eq. (4) may be corrected 
so as to restore the conservation of parallel momentum (which is violated by use of the Lorentz collision operator) either by solving a system of linear equations in which the coefficients are differently weighted energy moments of the mono-energetic transport coefficients [19-21] or by solving a generalised Spitzer problem accounting for an "effective" fraction of trapped particles derived from the mono-energetic quantity $D_{33}$ [21]. Thus, these coefficients represent a compact repository of pertinent information required for neoclassical transport calculations and provide a natural point of comparison for the various techniques used to solve the linearised drift kinetic equation.

Before proceeding further, it is appropriate to point out assumptions of the theory used here which are in some sense "stellarator-specific", in that they place restrictions on the theory's use for general toroidal devices (particularly axisymmetric tokamaks). Foremost is the assumption made in the derivation of eq. (1) that the lowest-order distribution function is a Maxwellian at rest in the laboratory frame, thereby precluding strong plasma rotation. Additionally, the $\mathbf{E}_{r} \times \mathbf{B}$ drift in the Vlasov operator is taken to be incompressible (with $\left\langle B^{2}\right\rangle$ appearing in the denominator instead of the correct $B^{2}$ so as to allow eq. (1) to remain conservative despite its reduced number of variables). Both of these assumptions are warranted in stellarators due to the unavoidable parallel viscosity caused by trapped particles and by the tendency for these devices to have large aspect ratios. For axisymmetric tokamaks these assumptions are often justified as well, and in such cases the mono-energetic transport coefficients employed here can be used to recover well-known results from tokamak neoclassical theory, including the intrinsic ambipolarity of the radial particle fluxes [19-21]. In the current work, however, tokamak results will appear only for reference purposes and, in particular, to furnish the analytic expressions used to normalise the mono-energetic coefficients.

To conclude this section, a short summary of the theoretical expectations concerning the mono-energetic transport coefficients will be presented. To this purpose, one begins by considering the simple model magnetic field

$$
B / B_{0}=1-\epsilon_{t} \cos \theta-\epsilon_{h} \cos (M \theta-N \phi),
$$

where $\theta$ and $\phi$ are the poloidal-angle and toroidal-angle coordinates, respectively, the term with magnitude $\epsilon_{t}=r / R_{0}$ is a consequence of the toroidal curvature and the term with magnitude $\epsilon_{h}=\epsilon_{h}(r)$ describes a helical magnetic field with multipolarity $M$ and field period number $N$. In spite of its simplicity, this model field contains all the ingredients necessary to define a number of terms and concepts used throughout the remainder of this paper. Of elementary importance is the fact that any variation of $B$ along field lines leads to reflection/trapping of particles with small parallel velocities. In axisymmetric tokamaks (e.g. the model field with $\epsilon_{h}=0$ ), the vertical drift of these trapped particles results in "banana" orbits with widths a significant fraction of the poloidal gyroradius but which, in the absence of collisions, experience no net radial displacement on average over the course of their periodic bounce 
motion as bananas spend equal amounts of time above and below the poloidal midplane. In the long-mean-free-path (lmfp) "banana-regime", the portion of $\widehat{f}_{I I}$ responsible for radial transport (symmetric in the pitch-angle variable $p$ ) is thus inversely proportional to $\lambda^{\star}$ [22] and hence $D_{11} \propto \nu\left(v_{d} R_{0} / v\right)^{2}$. Approximating the velocity dependence of the collision frequency by $\nu \propto v^{-3}$, one obtains $D_{11} \propto v^{-1}$ and thermal transport coefficients $L_{i j} \propto T^{-1 / 2}$ for $i, j=1,2$.

Banana orbits also exist in stellarators, but of far more concern are the orbits of particles trapped in the helical variation of $B$ which remain highly localised in poloidal angle over the course of a bounce so that the radial component of their vertical drift is non-zero on average. Given such orbits, the assumption of radially local transport remains warranted only if the poloidal $\mathbf{E}_{r} \times \mathbf{B}$ precession frequency of particles trapped in helical ripples is large compared with $v_{d} / r$ or if pitch-angle scattering is frequent enough to limit the time particles remain localised by collisionally removing them from the ripple. In the latter case (typical for electrons in high-temperature stellarator plasmas), the symmetric portion of $\widehat{f}_{I I}$ depends linearly on the normalised mean-free-path [6,7] which leads to $D_{11} \propto v_{d}^{2} / \nu \propto v^{7}$ and $L_{i j} \propto T^{7 / 2}$ for $i, j=1,2$. Where this result holds, particles are said to be in the " $1 / \nu$ regime", an obvious reference to the scaling of $D_{11}$ with collision frequency. The very unfavourable temperature dependence of the radial transport coefficients in this regime has prompted numerous efforts to optimise the magnetic fields of stellarators so as to reduce the geometrical factor associated with $1 / \nu$ losses; various strategies for doing so are discussed in Section 4 of this paper.

When the electric field is responsible for limiting the radial excursion of localised particles, theoretical solutions of eq. (3) have been derived assuming that trapping/detrapping of localised particles takes place either due to collisions or drifts. In the case of collisions, the symmetric portion of $\widehat{f}_{\text {II }}$ scales as $\left(v_{E}^{\star}\right)^{-3 / 2}\left(\lambda^{\star}\right)^{-1 / 2}$ which yields $D_{11} \propto v_{d}^{2}\left(R_{0} B_{0} / E_{r}\right)^{3 / 2} \nu^{1 / 2}$ and $L_{i j} \propto$ $T^{5 / 4} E_{r}^{-3 / 2}$ for $i, j=1,2[6]$; this is commonly referred to as the " $\sqrt{\nu}$ regime". When drifts are invoked the scaling of the distribution function becomes $\left(v_{E}^{\star}\right)^{-2}\left(\lambda^{\star}\right)^{-1}$ resulting in $D_{11} \propto$ $\left(v_{d} R_{0} B_{0} / E_{r}\right)^{2} \nu$ and $L_{i j} \propto T^{1 / 2} E_{r}^{-2}$ for $i, j=1,2$ [23]; this is the so-called " $\nu$ regime". The dependence of these transport coefficients on the radial electric field combined with the ambipolarity condition on the radial particle fluxes, $\sum_{\alpha} q^{\alpha} I_{1}^{\alpha}=0$, leads to a non-linear equation which can have multiple solutions for $E_{r}$ [24]. This is a feature of $\operatorname{lmfp}$ neoclassical transport theory in stellarators which has no counterpart for axisymmetric tokamaks.

With regard to the parallel transport the differences between stellarators and tokamaks are much less dramatic. Regardless of the magnetic configuration, the antisymmetric portion of $\widehat{f}_{I}$ is linearly proportional to the normalised mean-free-path and independent of the radial electric field for all collision frequencies [25], yielding $D_{33} \propto v^{2} / \nu$ and $L_{33} \propto T^{5 / 2}$. Also regardless of the configuration, $D_{33}$ is reduced when reflected particles exist but in this respect stellarators are affected at somewhat larger values of $\nu$ due to the higher bounce frequencies of localised particles compared with tokamak bananas. Further, assuming stellarator and tokamak have identical values of $\epsilon_{t}$, it is evident from eq. (5) that the stellarator will have a larger fraction of 
trapped particles and thus smaller values of $D_{33}$ in the collisionless limit.

To obtain the last of the three mono-energetic transport coefficients, needed to determine the bootstrap current (or the Ware pinch), one must either solve eq. (2) for the symmetric portion of $\widehat{f}_{I}$ or eq. (3) for the antisymmetric portion of $\widehat{f}_{I I}$. Only the trivial solution exists in the limit of small $\lambda^{\star}$ as the inhomogeneous term of each differential equation has the opposite symmetry of the portion of the distribution function being sought and hence $D_{31}=0$. In the opposite limit $\left(\lambda^{\star} \rightarrow \infty\right)$ the relevant portion of the distribution function becomes independent of the normalised mean-free-path [26] so that $D_{31} \propto v_{d} R_{0}$ and $L_{3 j} \propto T$ (for $j=1,2$ ) for both stellarators and tokamaks. The geometrical factors, on the other hand, can be quite different as the bootstrap current coefficient is of opposite sign in the axisymmetric $\left(\epsilon_{h}=0\right)$ and helically symmetric $\left(\epsilon_{t}=0\right)$ limits of eq. (5) for conventional helical windings with $M_{t} / N<1$, where $t$ is the rotational transform value of the flux surface under consideration. It is thus conceivable in a stellarator to make $D_{31}$ vanish by an appropriate combination of toroidal curvature and helical variation of $B$. Finally, in the stellarator $\operatorname{lmfp}$ regime the predominant portion of $\widehat{f}_{I I}$ is symmetric in $p$ and strongly dependent on the radial electric field (in contrast to axisymmetric tokamaks for which $\widehat{f}_{I I}$ is predominantly antisymmetric and independent of $E_{r}$ ) which makes it plausible that the antisymmetric portion of $\widehat{f}_{I I}$ will also depend on $E_{r}$ due to the coupling of symmetric and antisymmetric terms in the kinetic equation through the Vlasov and Lorentz operators. This would argue for a dependence of $D_{31}$ on the value of $v_{E}^{\star}$ in the stellarator $\operatorname{lmfp}$ regime which is lacking for tokamaks although analytic predictions concerning this dependence have yet to be formulated.

\section{Numerical Methods Used to Determine Mono-Energetic Transport Coefficients}

Although analytic solutions of the kinetic equation provide useful physical insight into the neoclassical transport processes in stellarators, they are usually incapable of providing accurate values for relevant geometrical factors and are therefore of limited help when it comes to practical tasks such as comparing experimental results with neoclassical expectations or performing predictive simulations of high-temperature stellarator plasmas. For these purposes, mono-energetic transport coefficients calculated using numerical methods are preferable since they can be determined in all parameter ranges of interest (and not only for a particular ordering of frequencies appearing in the kinetic equation) and for arbitrarily complex magnetic field structure. The latter point is of particular relevance for those configurations considered here which are characterised by strong 3-D plasma shaping achieved through the use of modular coils.

The earliest numerical tools for investigating neoclassical transport in stellarators evolved 
from codes written to follow particle orbits in magnetic and electric fields [27,28]. Mathematically, such orbits represent solutions of the Vlasov equation obtained by the method of characteristics; an extension of this approach to treat the drift kinetic equation is thus largely a matter of developing numerical algorithms to simulate the effects of the collision operator. When the algorithm makes use of random numbers it is commonly referred to as a "Monte Carlo method" to indicate the element of chance involved.

By considering an ensemble of mono-energetic test particles, an orbit-following Monte Carlo code can be used to obtain a numerical estimate of $D_{11}$ if the simulation parameters are chosen to insure that particles deviate little from their original flux surface, $r_{0}$. This is best achieved by considering particles with extremely small gyroradii and afterwards scaling the results to arbitrary values of gyroradius. Mathematically this may be viewed as a numerical solution of the continuity equation describing the radial diffusion of a particle "density" initially characterised by a delta function located at $r=r_{0}$. Assuming sufficient radial localisation the solution of the continuity equation is known to be Gaussian and a numerical estimate of the diffusion coefficient can be obtained from the simulation particles' dispersion $[28,29]$ once they have been followed for a time sufficient to eliminate any effects which the particles' "initial values" might have on the results (a minimum of one collision time is mandatory).

The results from three Monte Carlo codes [10-12] which perform simulations of this type are included in the ICNTS benchmarking presented in Section 5. All three employ Boozer flux coordinates [30] which greatly simplifies the description of particle trajectories for given magnetic and electric fields. The codes are similar in other respects as well, differing mainly in numerical details affecting how the integration of particle orbits is carried out. Given these similarities, it was not considered necessary to apply each of the codes to every configuration examined here; once the three had been successfully benchmarked for a handful of cases it was deemed sufficient to treat the remaining configurations with a single code, or with two at most. This decision was taken to conserve computational resources as the cost of Monte Carlo calculations increases linearly with the collision time, making each step further into the $\operatorname{lmfp}$ regime increasingly expensive. This demand on computational resources may be considered a general disadvantage of orbit-following Monte Carlo codes but for the simulations carried out here it was also observed that the radial distribution of particles becomes non-Gaussian above some critical value of normalised mean-free-path, with large radial displacements more common than would be expected. This indicates that non-local transport is present in the simulations $[31,32]$ and that the numerical scheme is no longer appropriate for determining $D_{11}$ when $\lambda^{\star}$ exceeds this critical value.

Although an orbit-following Monte Carlo code has also been used in the past to estimate the bootstrap current in stellarators [33], this method has not been applied in the ICNTS benchmarking to determine either $D_{31}$ or $D_{33}$. This is simply due to a lack of numerical candidates for such calculations as recent developments of Monte Carlo methods to determine 
these two mono-energetic transport coefficients have relied almost exclusively on the so-called $\delta f$ approach described in the following.

Another numerical means of determining the mono-energetic transport coefficients is the direct time integration of eqs. (2) and (3) for an ensemble of mono-energetic simulation particles (more commonly referred to in the literature as "markers") with the effects of the Lorentz collision operator again described using Monte Carlo methods. This yields numerical representations for the solutions

$$
\left[\begin{array}{c}
\widehat{f}_{I} \\
\widehat{f}_{I I}
\end{array}\right]=\sum_{i}\left[\begin{array}{l}
w_{I} \\
w_{I I}
\end{array}\right]_{i} \delta\left(\mathbf{X}-\mathbf{X}_{i}\right)
$$

where $i$ is the particle index, $\mathbf{X}$ represents the three phase-space variables and the "weights"

$$
w_{I}=-\frac{v}{R_{0}} \int \mathrm{d} t p \frac{B}{B_{0}} \quad w_{I I}=-\frac{v}{R_{0}} \int \mathrm{d} t \frac{1}{v_{d}} \frac{\mathrm{d} r}{\mathrm{~d} t}
$$

will be recognised as the time integrals of the inhomogeneous terms appearing in the dimensionless drift kinetic equations. Numerical schemes of this type are referred to as $\delta f$ Monte Carlo methods [13-15] to indicate that (non-Maxwellian) perturbed portions of the distribution function are being solved for, from which numerical estimates of the mono-energetic transport coefficients are obtained in a straightforward manner. Non-local contributions to the transport cannot arise using this approach as all simulation particles are strictly confined to the flux surface of interest with $\mathrm{d} r / \mathrm{d} t$ only affecting their weights $w_{I I}$. The convergence properties of $\delta f$ Monte Carlo estimates for $D_{33}$ and $D_{11}$ are satisfactory due to the optimal weightings of $w_{I}$ and $w_{I I}$ for describing the parallel and radial transport, respectively, and required simulation times for a given statistical accuracy are directly proportional to $\lambda^{\star}$ as is the case for calculations of $D_{11}$ with orbit-following Monte Carlo codes. These weightings are non-optimal, however, when it comes to determining the bootstrap current coefficient in stellarators (or its Onsager conjugate, the Ware pinch coefficient) as the variance of statistical estimates for $D_{31}$ increases as the square of the mean free path [15], an effect which is commonly counteracted by increasing the number of simulation particles [34] (the standard error remains unchanged if the ratio of variance to number of simulation particles is held constant); the required simulation time for a given statistical accuracy is thus a cubic function of $\lambda^{\star}$ when the conventional marker weightings are employed [15]. The Monte Carlo results presented here for the bootstrap current coefficient counteract this statistical degradation either by a "filtering" of weights as is done in the VENUS $+\delta f$ code [14] or by employing an "advanced-weighting" technique [15]. In the latter case it has been demonstrated that the simulation-time scaling improves to $\left(\lambda^{\star}\right)^{3 / 2}$ which nevertheless indicates that computational costs of $D_{31}$ using $\delta f$ Monte Carlo codes will exceed those of $D_{11}$, with the difference widening as the collision frequency of the simulations is reduced. 
The Drift Kinetic Equation Solver (DKES) $[16,17]$ uses a variational approach to solve eqs. (2) and (3), in which the distribution function is expressed as a truncated FourierLegendre series (Fourier harmonics for the poloidal-angle and toroidal-angle dependence and Legendre polynomials to describe the pitch-angle dependence) and the resulting system of linear equations is solved using standard library routines. With the magnetic field also specified in terms of a Fourier series, the orthogonality properties of Fourier harmonics and Legendre polynomials make it a simple task to determine DKES results for the three mono-energetic transport coefficients. For the calculations presented here, the minimising and maximising variational principles have been invoked to obtain upper and lower bounds on the transport coefficients [17]. The convergence of these bounds is most strongly dependent on the value of $\lambda^{\star}$ (worsening as the normalised mean-free-path increases) but is also affected by the complexity of $B$ and the value of $v_{E}^{\star}$. Computational time for DKES increases nearly cubically with the number of Fourier modes used to describe the distribution function and linearly with the number of Legendre polynomials but convergence of the bounds is a very weak function of these two numbers in the $\operatorname{lmfp}$ regime. This is due to the increasing localisation of the perturbed distribution function at the phase-space boundary separating trapped and untrapped particles, which is poorly resolved using Fourier-Legendre test functions. Computational resources have limited the DKES calculations presented here to less than 2000 Fourier modes and 300 Legendre polynomials, which does not allow satisfactory convergence for some of the most collisionless cases investigated.

To reduce the cost in computational resources, efficient methods for solving "simplified" kinetic equations have also been developed and two such approaches are included here in the benchmarking. In the first approach, eqs. (2) and (3) are solved ignoring the $\mathbf{E}_{r} \times \mathbf{B}$ drift in the Vlasov operator, making it possible to determine the mono-energetic transport coefficients by performing properly weighted integrals along a field line of "infinite" length (i.e. sufficiently long to cover the magnetic flux surface). A numerical implementation of this field-line-integration technique is at the heart of all versions of the NEO code, which differ principally in their treatment of collisions. (These versions of NEO are not to be confused with another code of the same name recently developed for calculations of neoclassical transport in axisymmetric tokamaks [35].) In NEO-2 [9], for example, an adaptive third-order conservative finite-difference scheme is employed to properly resolve the effects of pitch-angle scattering within each of the boundary layers which form when a local maximum of $B$ is encountered along the field line. Alternately, extremely efficient calculations are possible in the collisionless limit making use of the asymptotic behaviour of the solution as was done in the original NEO [8] which is commonly used in stellarator optimisation packages to determine the level of $1 / \nu$ transport for a given magnetic field [36]. Both versions of NEO can deal with arbitrarily complex $B$ and convergence problems do not arise so that the codes' only limitation, the inability to describe the influence of $E_{r}$ on the transport, is due to the initial simplification 
of the Vlasov operator. The second approach, a numerical solution of the ripple-averaged kinetic equation, GSRAKE [18], does not suffer this shortcoming. The ripple average is a generalisation of the common bounce average (time average over the periodic motion of reflected particles) so as to encompass all of phase space (including passing particles) and is performed as a separation of time scales to eliminate the most rapidly varying spatial coordinate in the kinetic equation. This separation can only be carried out efficiently if the structure of $B$ is described accurately within the so-called multiple-helicity model [37] which GSRAKE employs in the construction of its coordinate system [38]. For the configurations considered within the ICNTS such a model field is often inadequate and limits the use of GSRAKE in the benchmarking to a handful of cases. When applicable, however, the method used to solve the kinetic equation - combining a Fourier expansion in the remaining angular coordinate and finite differencing in the pitch-angle variable — possesses both computational speed and excellent convergence even for extreme values of normalised mean-free-path.

\section{Configurations}

Although the stellarator is the oldest concept for magnetic confinement of a fusion plasma $[39,40]$, it has spent the great majority of its existence in the shadow of its toroidal cousin, the tokamak. This is in spite of the fact that confinement in stellarators has typically been at least as good as that observed in tokamaks of comparable size [2]. The principal reason for this neglect is certainly historical but stellarators are also perceived as having disagreeably complex coil systems and if the magnetic fields they produce also suffer from significant $1 / \nu$ transport and poor confinement of energetic particles it is difficult to imagine such a device as an economically attractive reactor. In defence of stellarator coil systems it must be said that their "complexity" is accompanied by undeniable advantages, foremost of which include the intrinsic capability of steady-state, disruption-free operation. With this in mind, considerable effort has been devoted during recent years to improving the equilibrium, stability and confinement properties of stellarator magnetic fields employing strong 3-D shaping of the plasma column made possible by non-planar modular coils [41]. These efforts have resulted in a number of optimisation strategies which share the common attribute of employing magnetic fields with a high degree of omnigeneity, meaning that they possess time-averaged drift surfaces - i.e. contours of constant $\mathcal{J}$, where $\mathcal{J}$ is the second (or longitudinal) adiabatic invariant of particle motion - which (nearly) coincide with their magnetic flux surfaces [42,43]. Such efforts are exemplified here by five configurations, among which are one device already in operation and a second currently under construction. The other four devices considered within the ICNTS fall into the categories of "classical" or "partially optimised" stellarators and are either active experiments or have been recently decommissioned. 
Beginning with this latter group, the Compact Helical System (CHS) [44], a low-aspect-ratio heliotron with $M=2$ and $N=8$, was in operation at the National Institute for Fusion Science (NIFS) in Nagoya and Toki, Japan, from 1988 until 2006. Contours of constant magnetic field strength for the standard vacuum configuration of this device are plotted in Figure 1. For CHS and all the subsequent configurations depicted in this section, $B$ has been calculated with the VMEC equilibrium code [45] and then decomposed in Boozer magnetic flux coordinates according to

$$
B(r, \phi, \theta) / B_{0}(r)=1+\sum_{n=1}^{\infty} b_{0, n}(r) \cos (n N \phi)+\sum_{m=1}^{\infty} \sum_{n=-\infty}^{\infty} b_{m, n}(r) \cos (m \theta-n N \phi)
$$

where $r$ remains the flux-surface label but $\phi$ and $\theta$ are now generalised angular coordinates commonly referred to as the Boozer toroidal and poloidal angles, respectively [28]. The flux surface at $\rho \equiv \sqrt{s}=r / a=0.5$ is shown, where $s$ is the normalised toroidal flux and $r=a$ defines the last closed flux surface of the equilibrium. In Boozer coordinates, field lines obey $\mathrm{d} \theta / \mathrm{d} \phi=t(r)$ and are thus "straight" with a slope given by the rotational transform of the flux surface. For the convention used here, the helical path of a field line rotates in a left-handed sense for positive values of the rotational transform while right-handed rotation is indicated by $t<0$. In the bottom portion of Figure 1 , the value of $B / B_{0}$ along the field line passing through $\phi=0$ and $\theta=0$ is plotted along a length corresponding to two poloidal circuits of the device; the rapid helical modulation and the slower toroidal variation of $B$ are easily identified.

A very similar magnetic field topology is exhibited in Figure $2 \mathrm{a}$ by the standard configuration of the Large Helical Device (LHD) [46], which is also a heliotron but with $M=2$, $N=10$ and helical windings which rotate in a left-handed sense as they encircle the torus instead of the right-handed rotation used for CHS. Since beginning operation at NIFS in 1998, LHD has been the world's largest stellarator, the vacuum configuration considered here having a major radius of $R_{0}=3.75 \mathrm{~m}$ and a minor radius of $a=0.56 \mathrm{~m}$. For both CHS and LHD, the Boozer representations of $B$ contain only a small number of harmonics with significant magnitudes. Indeed, the standard configurations of these two devices are approximated well by the simple model magnetic field of eq. (5). As heliotrons, this magnetic field structure can be altered to a certain degree by using the vertical field coils of each device to shift the plasma column in the radial direction. An example for LHD is given in Figure $2 b$ in which the magnetic axis has been shifted inwards to $R_{0}=3.6 \mathrm{~m}$. This results in a magnetic field topology in which deeply trapped particles encounter a nearly constant value of $B$ over the course of their bounce motion and thus experience rather small radial $\nabla B$ drift; this represents the simplest type of "drift optimisation" in stellarators [47] and is expected to significantly reduce $1 / \nu$ losses since the deeply trapped particles typically contribute disproportionately in this regime [37]. Improving the neoclassical confinement of heliotrons in this manner has been traditionally frowned upon, however, as an inward shift of the plasma column leads to a 

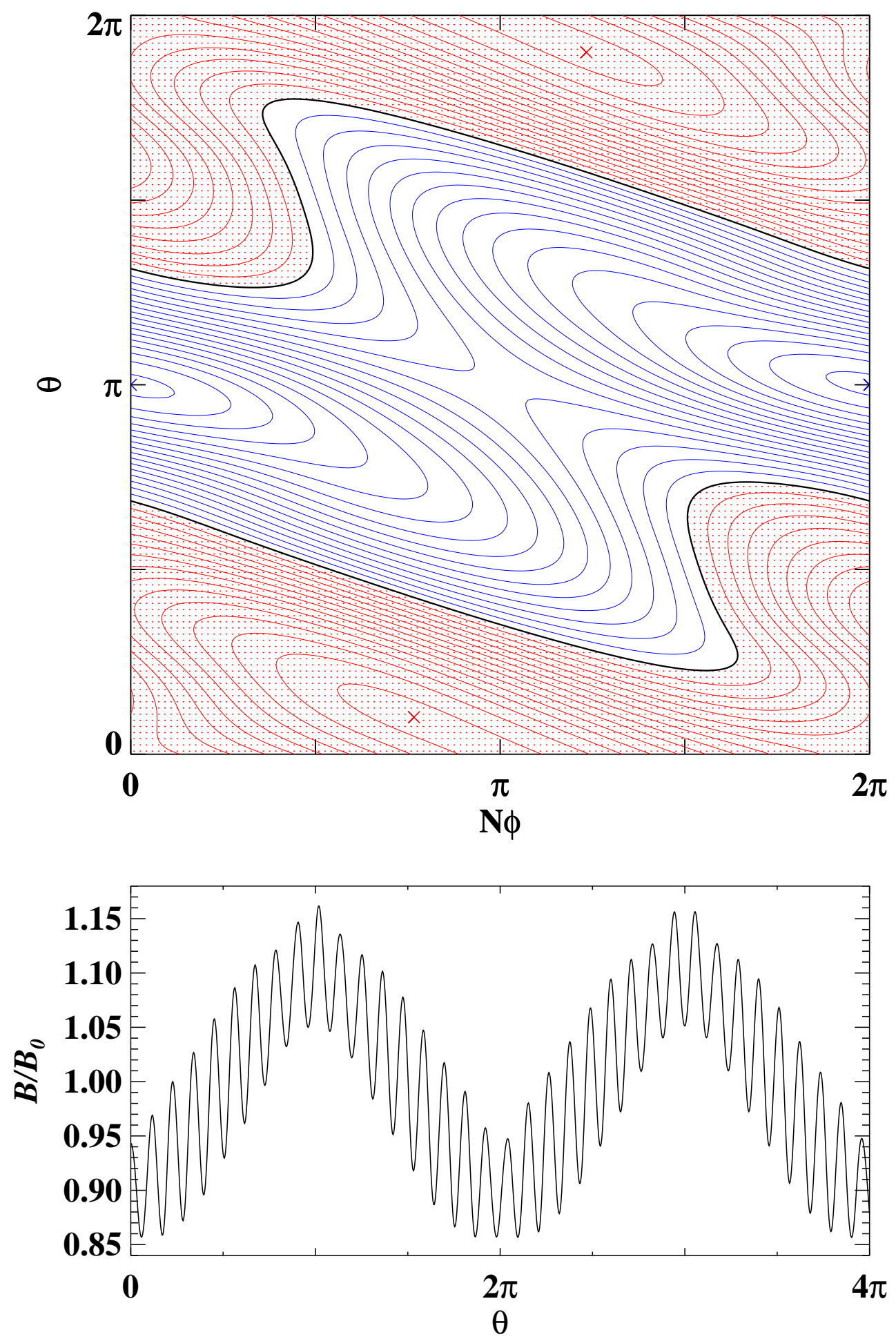

Figure 1. Contours of constant magnetic field strength for one field period of CHS (with $N=8$ ) are shown for the $\rho=0.5$ flux surface of the standard vacuum configuration which has $t=-0.4067$. The thick (black) contour denotes $B / B_{0}=1$, in the shaded region the (red) contours are at lower values, in the unshaded region the (blue) contours are at higher values. The contours are spaced at intervals of 0.01 . The locations of the absolute maximum and minimum values of $B$ on the surface are indicated by $\times$. In the lower frame, the magnitude of $B / B_{0}$ along the field line passing through $\phi=0$ and $\theta=0$ is plotted over two poloidal circuits of the torus. 

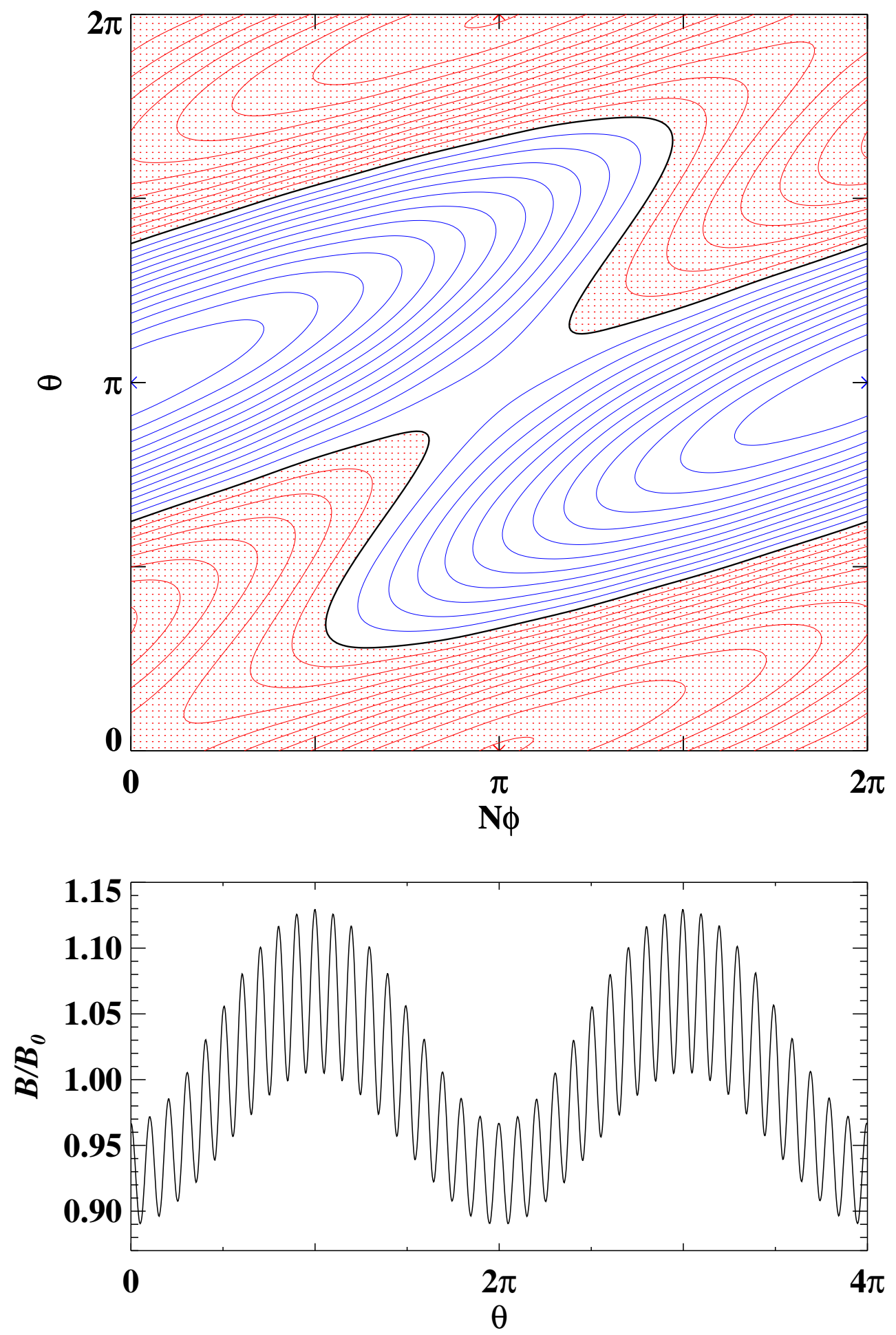

Figure 2a. Contours of constant magnetic field strength for one field period of LHD (with $N=10$ ) are shown for the $\rho=0.5$ flux surface of the standard vacuum configuration $\left(R_{0}=3.7481 \mathrm{~m}\right)$ which has $t=0.4542$. Contours have been plotted following the same scheme employed in Figure 1 . In the lower frame, the magnitude of $B / B_{0}$ along the field line passing through $\phi=0$ and $\theta=0$ is plotted over two poloidal circuits of the torus. 

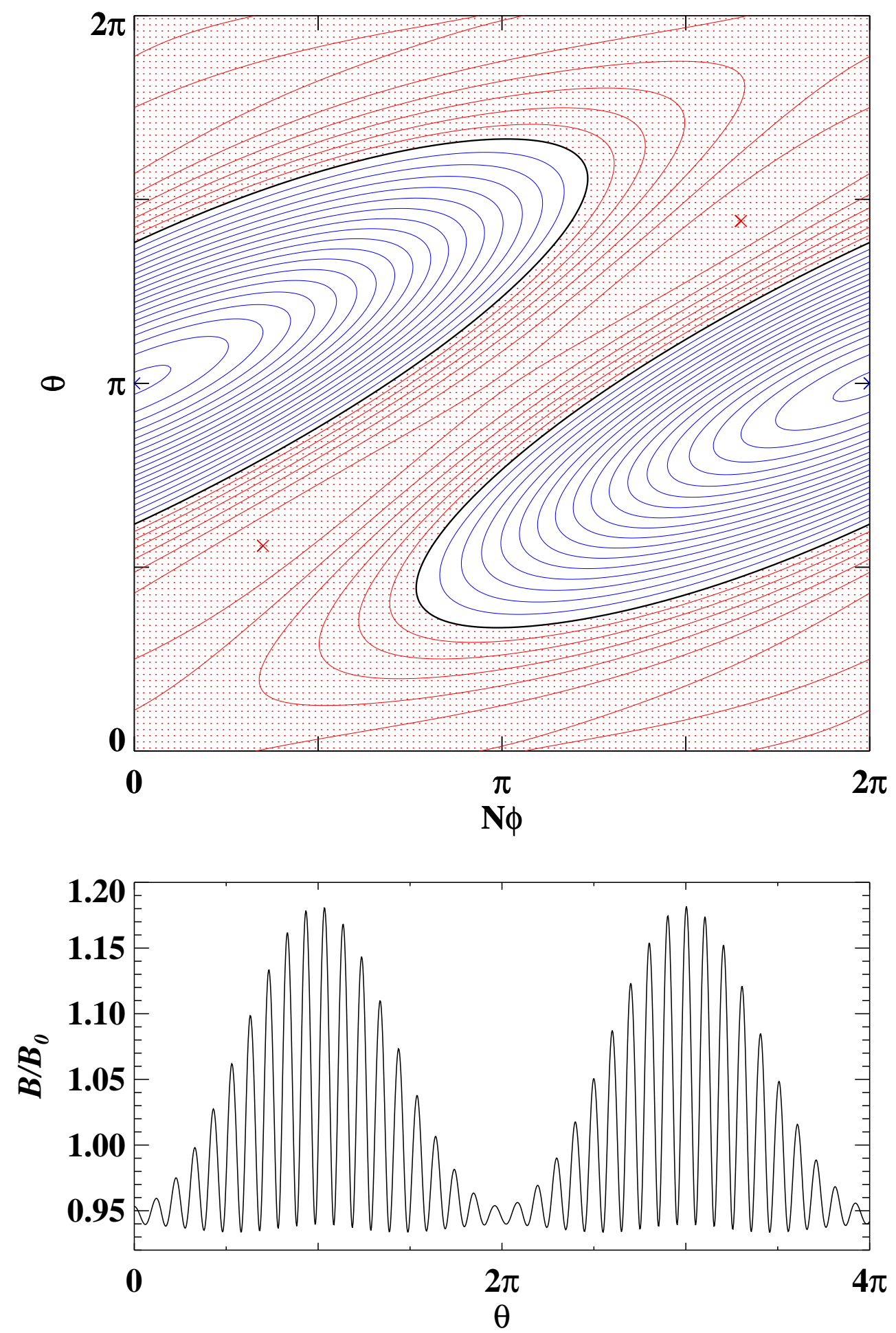

Figure 2b. Contours of constant magnetic field strength for one field period of LHD are shown for the $\rho=0.5$ flux surface of the inward-shifted vacuum configuration $\left(R_{0}=3.6024 \mathrm{~m}\right)$ which has $t=0.4692$. Contours have been plotted following the same scheme employed in Figure 1. In the lower frame, the magnitude of $B / B_{0}$ along the field line passing through $\phi=0$ and $\theta=0$ is plotted over two poloidal circuits of the torus. 
configuration with a vacuum magnetic hill which is predicted to be Mercier unstable for even weak pressure gradients. Experimentally, increased MHD activity has been observed in LHD for inward-shifted configurations but confinement is nevertheless superior to that observed in the standard or in outward-shifted configurations $[48,49]$. Similar results have been reported for CHS [50], leading to the conclusion that the Mercier stability criterion may be violated in heliotrons without serious consequences and thus making drift optimisation of this type a viable strategy for improving the neoclassical confinement. In this context, however, it should be noted that the Shafranov shift serves to displace the magnetic axis outwards so that the $1 / \nu$ transport of such an optimised vacuum configuration will always be degraded when the finite- $\beta$ equilibrium is considered [51].

A different type of optimisation was undertaken in the development of the "advanced stellarator" concept, which had Wendelstein 7-AS (W7-AS) as its prototype [52]. This device had a major radius of $R_{0}=2 \mathrm{~m}$, a minor radius of $a<0.2 \mathrm{~m}$ and was in operation at the Institute for Plasma Physics (IPP) in Garching, Germany, from 1988 until 2002. W7-AS was designed to improve on the MHD equilibrium and stability limits of classical low-shear stellarators (such as its predecessor Wendelstein 7-A), using non-planar modular coils to achieve an average "elongation" of its flux surfaces $\kappa=\left(\epsilon_{t} / b_{1,0}\right)^{2} \approx 2$. Such a reduction in the average toroidal curvature of $B$ is simultaneously of benefit with regard to neoclassical transport as it serves to decrease particles' radial drift velocity. Drift optimisation of deeply trapped particle orbits was not a goal of the W7-AS design, however, which is evident from the contour plot of $B$ shown in Figure 3 for the $t>1 / 3$ vacuum configuration of this device. Indeed, quite the contrary, deeply trapped particles find themselves localised in regions where $B$ varies considerably and this shortcoming is further exacerbated by the appearance of secondary minima of appreciable depth. As a consequence, the $1 / \nu$ transport of W7-AS was actually somewhat worse than that of the classical stellarator Wendelstein 7-A [3] (which also had $R_{0}=2 \mathrm{~m}$ but $\kappa \approx 1$ ).

MHD considerations also played a dominant role in the design of the heliac TJ-II [53], in operation at the CIEMAT laboratory in Madrid, Spain, since 1997. The coil system of TJ-II was chosen to allow considerable variation of the rotational transform and magnetic well depth to investigate their influence on the equilibrium and stability properties of the device but little attention was paid to the magnetic field topology other than to arrange the coils so as to minimise the variation of $B$ on the magnetic axis [54]. Contours of constant magnetic field strength for the standard vacuum configuration of TJ-II are plotted in Figure 4 and exhibit the most complicated structure of $B$ considered within the ICNTS. The deep, highly localised ripples of this field are particularly detrimental with respect to neoclassical transport; as will be seen in the next section, TJ-II has the largest $1 / \nu$ transport among the stellarators considered here.

As mentioned at the beginning of this section, the remaining stellarators investigated here were designed to achieve a high degree of omnigeneity and the configurations which resulted appear in the scientific literature accompanied by characterisations such as quasi-helically 

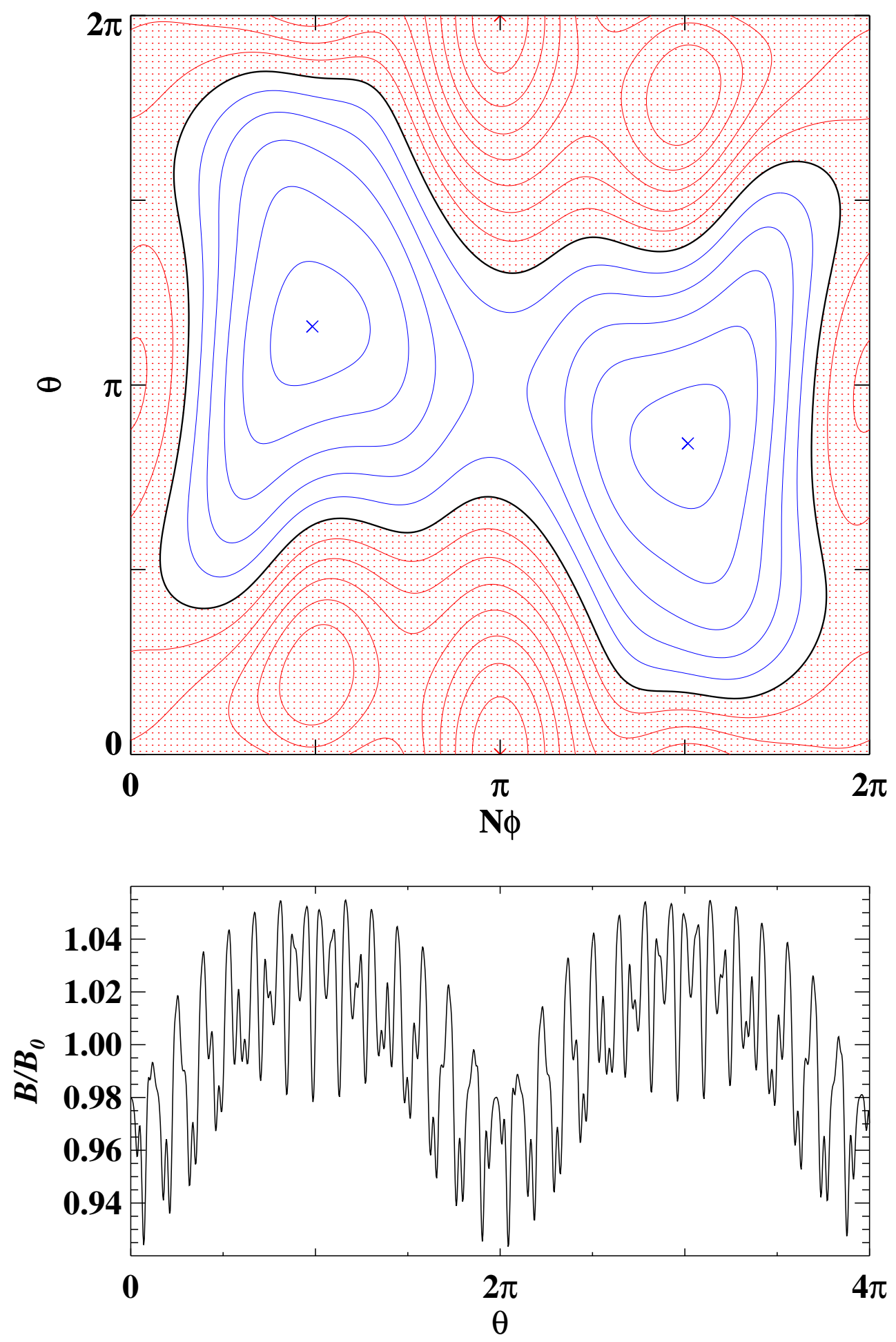

Figure 3. Contours of constant magnetic field strength for one field period of W7-AS (with $N=5$ ) are shown for the $\rho=0.5$ flux surface of a vacuum configuration which has $t=0.3525$. Contours have been plotted following the same scheme employed in Figure 1. In the lower frame, the magnitude of $B / B_{0}$ along the field line passing through $\phi=0$ and $\theta=0$ is plotted over two poloidal circuits of the torus. 

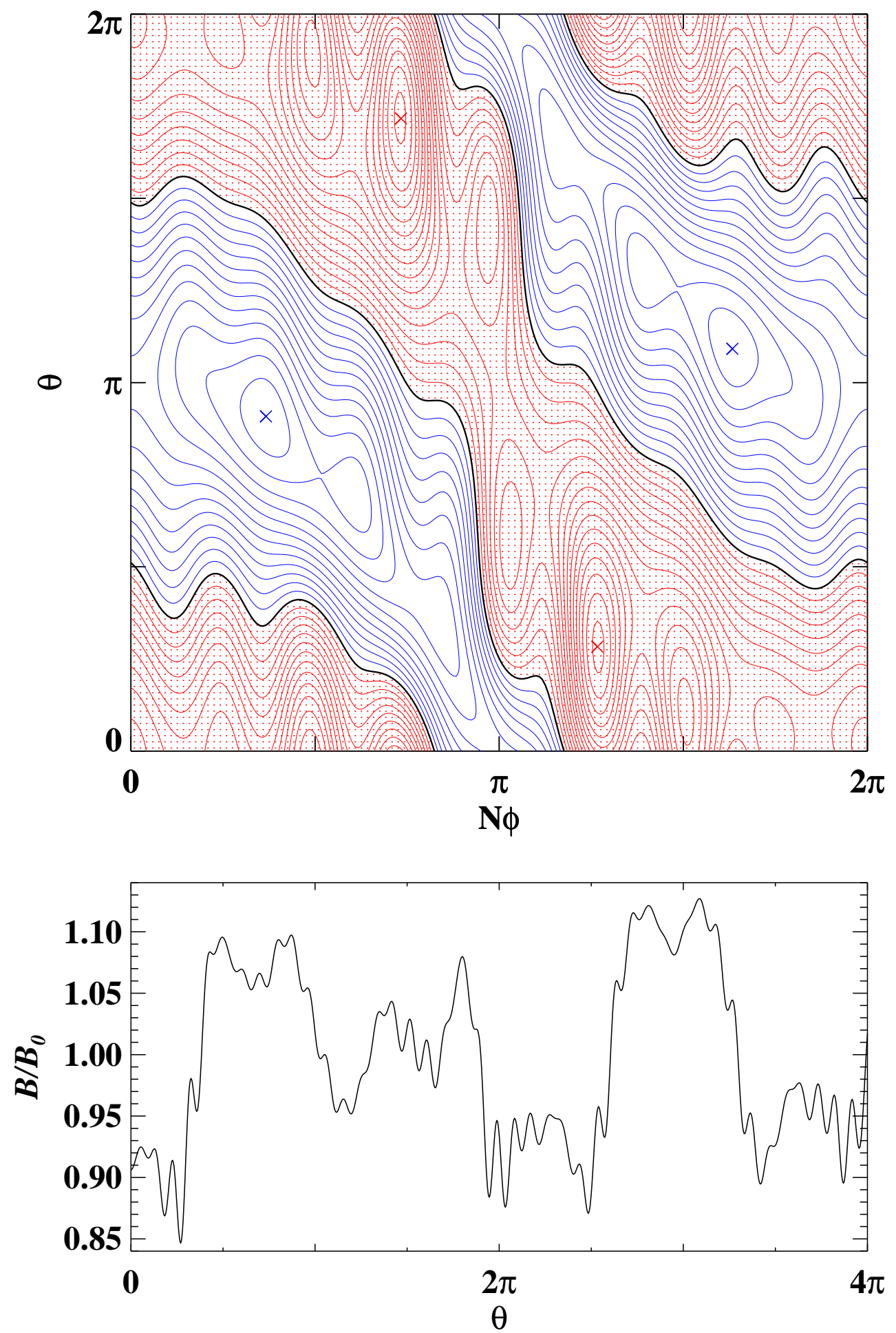

Figure 4. Contours of constant magnetic field strength for one field period of TJ-II (with $N=4$ ) are shown for the $\rho=0.5$ flux surface of the standard vacuum configuration which has $t=-1.4759$. Contours have been plotted following the same scheme employed in Figure 1. In the lower frame, the magnitude of $B / B_{0}$ along the field line passing through $\phi=0$ and $\theta=0$ is plotted over two poloidal circuits of the torus. 
symmetric, quasi-axisymmetric, quasi-poloidally symmetric and quasi-isodynamic. It will be noted that quasi is common to all of these designations but this adverb has been chosen by different authors to mean different things so that a brief digression will be helpful at this point to clarify the terminology. As originally defined, the concept of quasi-symmetry made use of the intuitively attractive observation that the structure of $B$ in magnetic coordinates is the quantity of relevance for determining particle trajectories and if this structure possesses a particular symmetry then its neoclassical transport properties will be identical with those of a device which has the same symmetry in real-space coordinates. Thus, an equilibrium with $B=B(r, \theta-N \phi)$ is quasi-helically symmetric [55] with neoclassical transport as in a straight helix (ignoring end losses), while $B=B(r, \theta)$ is said to be quasi-axisymmetric [56] with neoclassical transport as in the equivalent tokamak. The third conceivable quasi-symmetry, quasi-poloidal with $B=B(r, \phi)$, does not exist in a strict sense as its real-space equivalent, a simple (straight) mirror, has guiding centre drift trajectories which never leave their flux surfaces so that neoclassical radial transport and bootstrap current both vanish. At finite aspect ratio, toroidal equilibria which have zero neoclassical transport cannot exist [57] so that quasipoloidal symmetry [36] can hold only in the 'weak' sense of describing equilibria for which $b_{0, n}$ harmonics are dominant in the Boozer representation of $B$. More precise is the designation quasi-isodynamic which is used to denote omnigenous equilibria with a large fraction of trapped particles for all flux surfaces (including the magnetic axis) which experience only slow poloidal drift, on average, during the course of their bounce motion [58]. Here, quasi is used to signify a relaxation of the strict demands on truly isodynamic equilibria [59] in which even the instantaneous particle drift off a field line is directed only poloidally.

Although exact quasi-symmetry is not possible [60], approximations thereof are attainable to a degree comparable with what is achieved in tokamaks when the ripple due to the finite extent of the toroidal field coils is accounted for. An example of such a device is the (quasi-) Helically Symmetric Experiment (HSX) [61] depicted in Figure 5, which has been in operation at the University of Wisconsin-Madison, U.S.A., since 1999. Although the surface shown has an inverse aspect ratio of $\epsilon_{t}=0.0483$, its toroidal curvature in Boozer coordinates is more than two orders of magnitude smaller and is thus indiscernible with the naked eye. The visible departure from quasi-helical symmetry is instead largely due to the coil ripple produced by the twelve modular coils in each field period. In experiments, the improvement in confinement for thermal and fast particles predicted for quasi-helical symmetry has been demonstrated in HSX by also considering discharges in which the magnetic configuration has been altered to purposely spoil the quasi-symmetry $[62,63]$.

An example of quasi-axisymmetry is provided by the magnetic field of the National Compact Stellarator Experiment (NCSX) [64], shown in Figure 6 for a standard equilibrium with a plasma current of $I_{p}=174 \mathrm{kA}$ and a volume-averaged $\beta$ of $4.1 \%$ [65]. The value of $I_{p}$ assumed for this equilibrium is on the order of the expected bootstrap current and serves to 

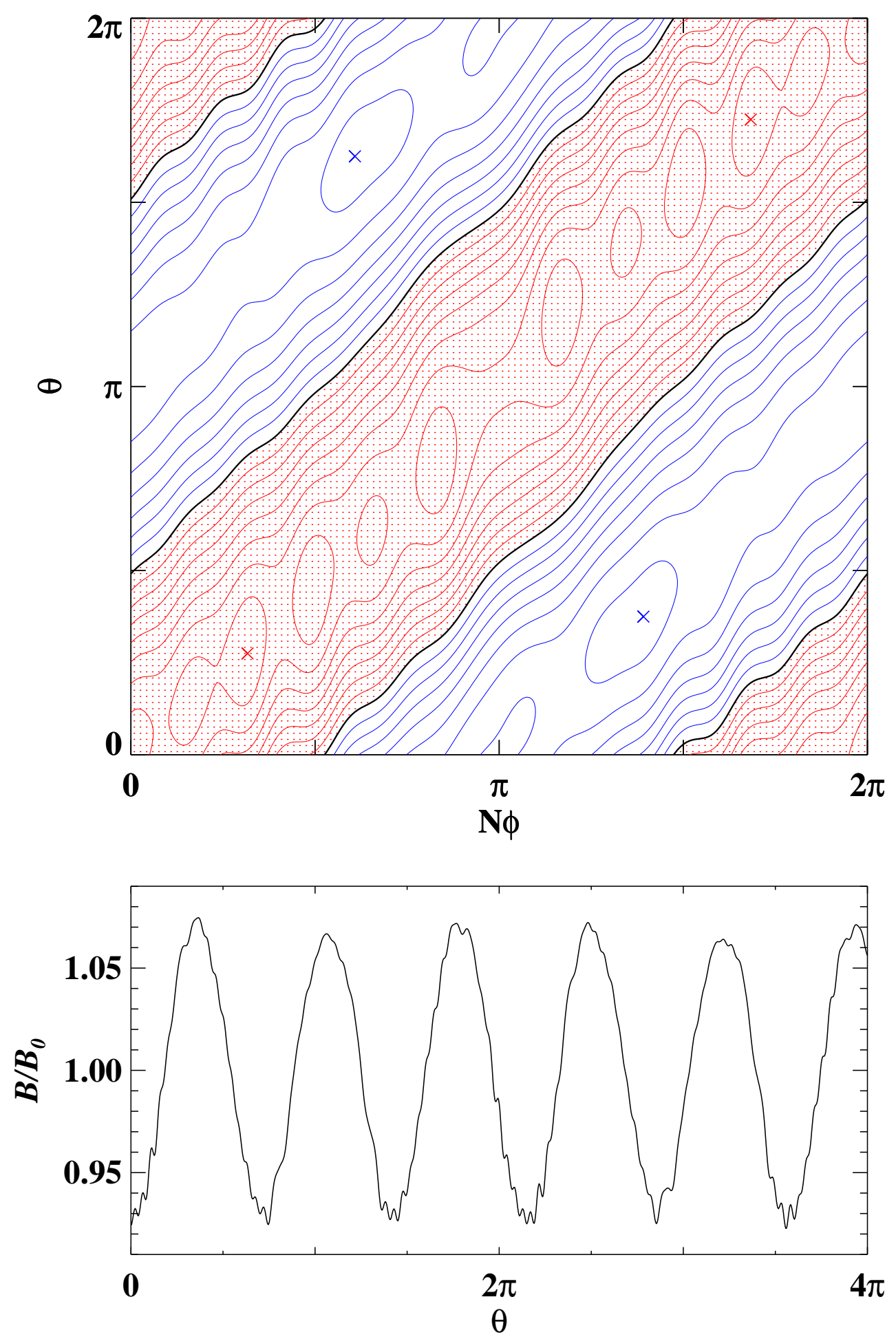

Figure 5. Contours of constant magnetic field strength for one field period of HSX (with $N=4$ ) are shown for the $\rho=0.5$ flux surface of the standard vacuum configuration which has $t=1.0537$. Contours have been plotted following the same scheme employed in Figure 1. In the lower frame, the magnitude of $B / B_{0}$ along the field line passing through $\phi=0$ and $\theta=0$ is plotted over two poloidal circuits of the torus. 

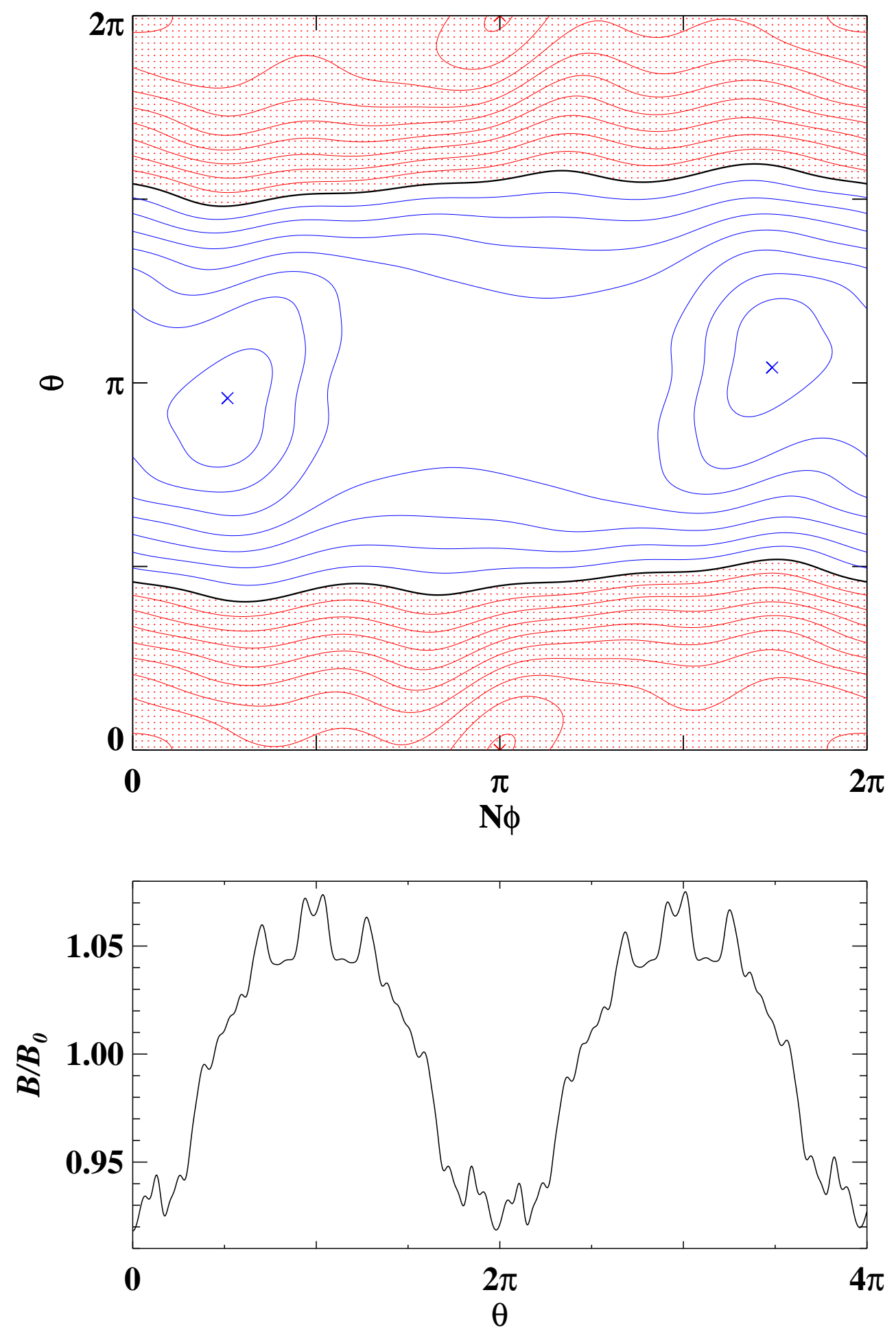

Figure 6. Contours of constant magnetic field strength for one field period of NCSX (with $N=3$ ) are shown for the $\rho=0.5$ flux surface of the reference S3 plasma configuration with $I_{p}=174 \mathrm{kA}$ and $<\beta>=4.1 \%$ [65] which has $t=0.4942$. Contours have been plotted following the same scheme employed in Figure 1. In the lower frame, the magnitude of $B / B_{0}$ along the field line passing through $\phi=0$ and $\theta=0$ is plotted over two poloidal circuits of the torus. 
supply roughly $30 \%$ of the rotational transform. Such an increase in $t$ due to the tokamaklike nature of the bootstrap current in a quasi-axisymmetric device is perhaps to be preferred over the reduction which helical or quasi-helical symmetry leads to but neither case offers a straightforward solution to the problems of particle and energy exhaust in reactor-grade plasmas. Divertor research is still in its infancy in stellarators and experimental results exist only for the "island" divertor concept of W7-AS and the "helical" divertor of LHD [66]. The first of these makes use of a naturally occurring island chain with $t=N / M_{a}$ (where $M_{a}$ is an appropriate integer specific to the configuration) situated at the edge of the confinement volume to channel outward-flowing plasma into specially prepared divertor regions where recycling and exhaust are to take place. To perform this function properly, the position and size of the islands must conform closely with the design values chosen for the divertor. This was relatively straightforward in W7-AS, using an ohmic transformer to compensate modest levels of bootstrap current, thus maintaining the vacuum island structure by means of net-current-free operation. An island divertor accounting for large plasma current is also conceivable but would require a considerably more sophisticated procedure than has been experimentally demonstrated to date. In this respect, the helical divertor inherent to the high-shear scrape-off layer of heliotrons has an advantage as its physical basis, the strong stochastization of the layer due to multiple overlapping island chains, is rather insensitive to the equilibrium established in the core region. Unfortunately, construction of NCSX at the Princeton Plasma Physics Laboratory in the U.S.A. was essentially terminated in 2008 (although some testing of the coils was undertaken) and it is currently uncertain whether this device will ever begin plasma operation and have the opportunity to develop a viable divertor concept for low-shear quasi-symmetric devices with large bootstrap current.

Quasi-isodynamicity is a concept more amenable to use of the island divertor as it is consistent with a suppression of the bootstrap current while simultaneously reducing radial transport to an acceptably low level [67]. The magnetic-field-strength contours of two such equilibria are given here — the standard vacuum configuration of the Quasi-Poloidal Stellarator (QPS) [68], proposed by Oak Ridge National Laboratory, U.S.A., as a concept-exploration experiment is shown in Figure 7 and a Quasi-Isodynamic equilibrium with Poloidally Closed contours of the magnetic field strength (QIPC) [69] is depicted in Figure 8. The stronger variation of $B$ with poloidal angle in the case of QPS is largely due to its small aspect ratio $R_{0} / a=2.65$ in comparison with $R_{0} / a=11.5$ for QIPC. Both configurations have a fractionof-trapped-particles well in excess of $50 \%$ but nevertheless fulfil the requirement of small radial transport coefficients in the $\operatorname{lmfp}$ regime as will be seen in the next section.

The final configuration investigated within the ICNTS is the Wendelstein 7-X (W7-X) device [70,71], which is currently under construction at IPP in Greifswald, Germany. This helias (helical-axis advanced stellarator) [72] emerged from an integrated design process which had the goal of finding magnetic fields which simultaneously fulfil a number of optimisation 

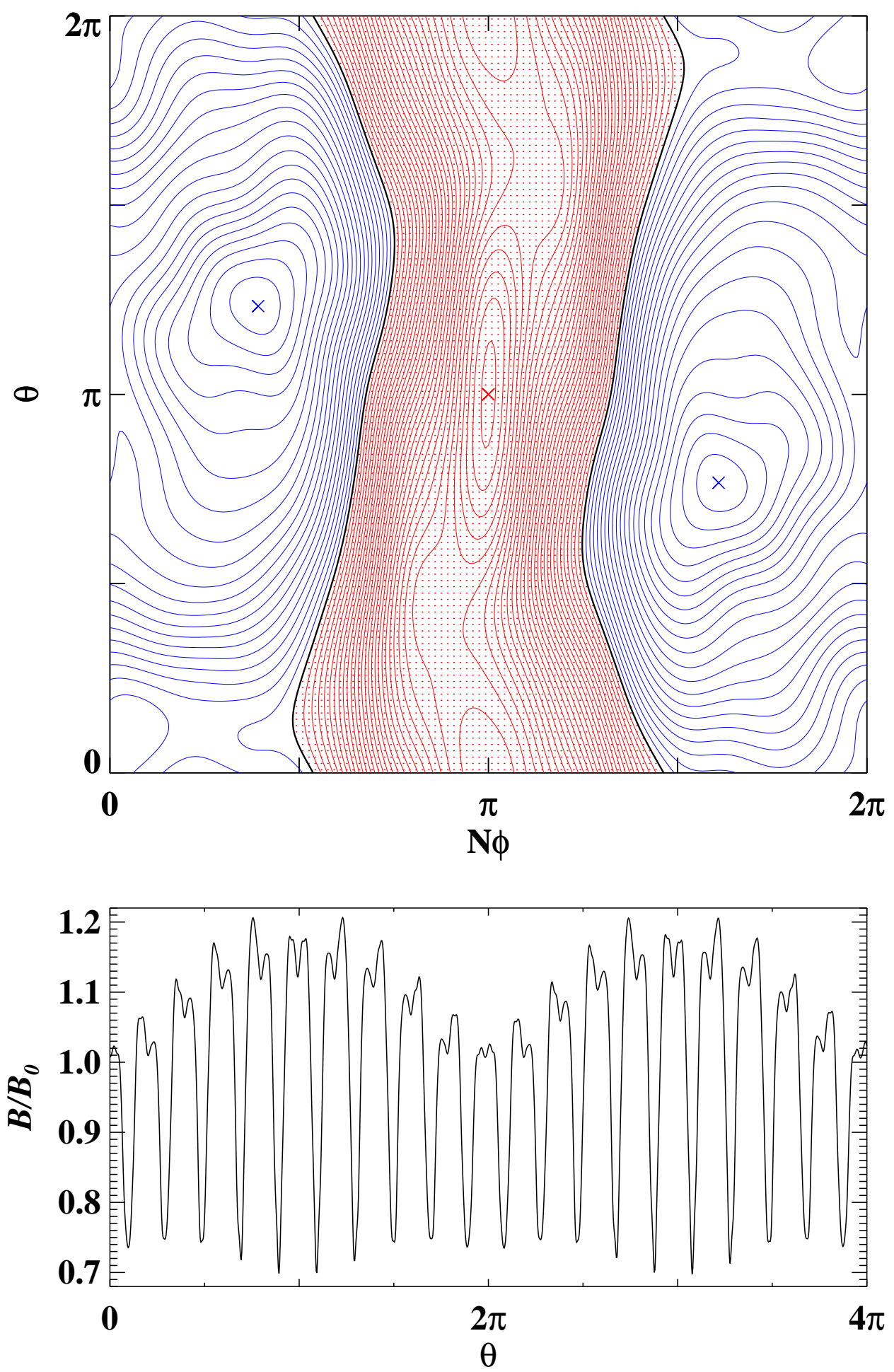

Figure 7. Contours of constant magnetic field strength for one field period of QPS (with $N=2$ ) are shown for the $\rho=0.5$ flux surface of the standard vacuum configuration which has $t=0.1985$. Contours have been plotted following the same scheme employed in Figure 1. In the lower frame, the magnitude of $B / B_{0}$ along the field line passing through $\phi=0$ and $\theta=0$ is plotted over two poloidal circuits of the torus. 

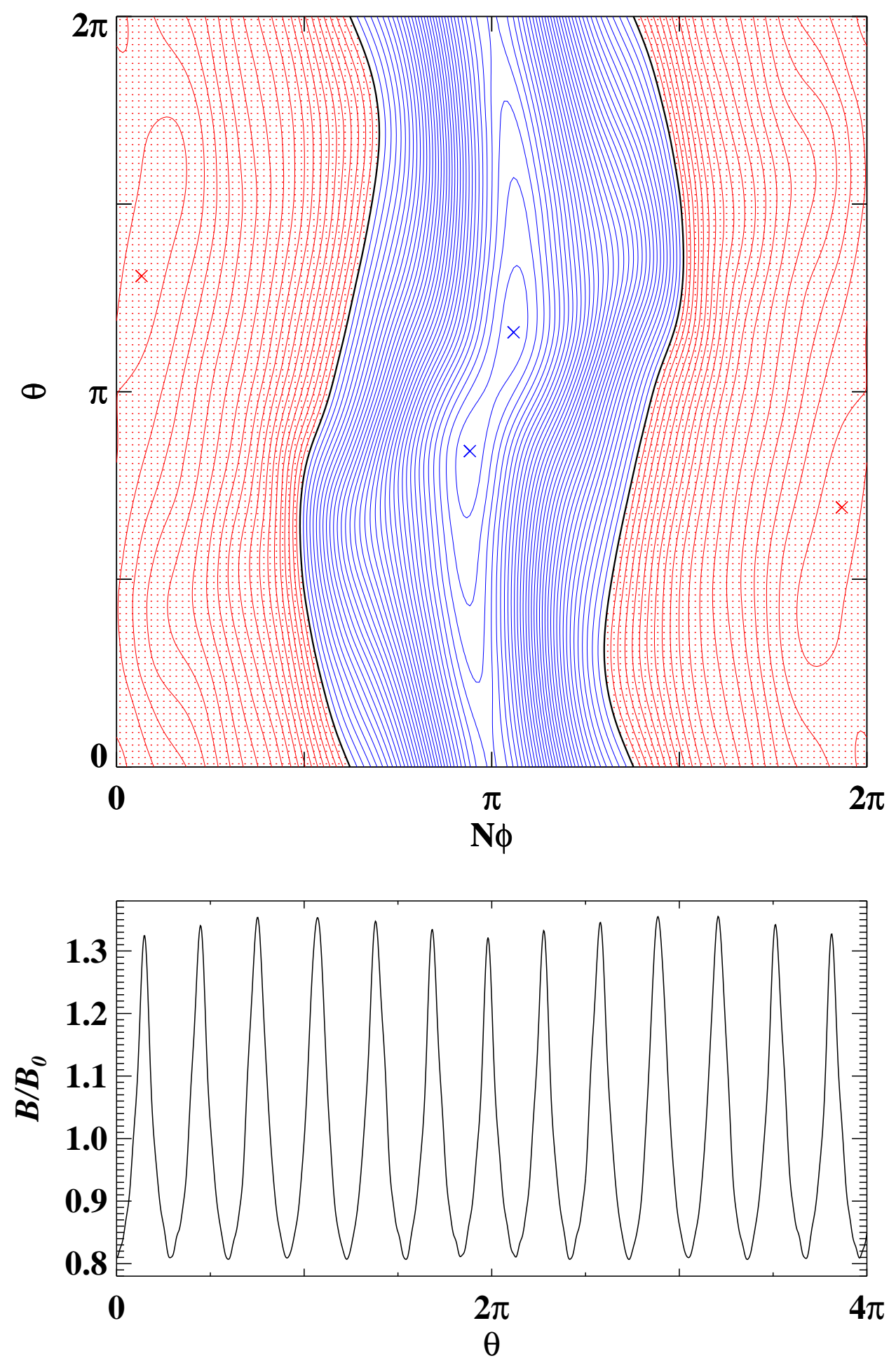

Figure 8. Contours of constant magnetic field strength for one field period of a QIPC equilibrium (with $N=6$ ) are shown for the $\rho=0.5$ flux surface of a vacuum configuration which has $t=0.9137$. Contours have been plotted following the same scheme employed in Figure 1. In the lower frame, the magnitude of $B / B_{0}$ along the field line passing through $\phi=0$ and $\theta=0$ is plotted over two poloidal circuits of the torus. 

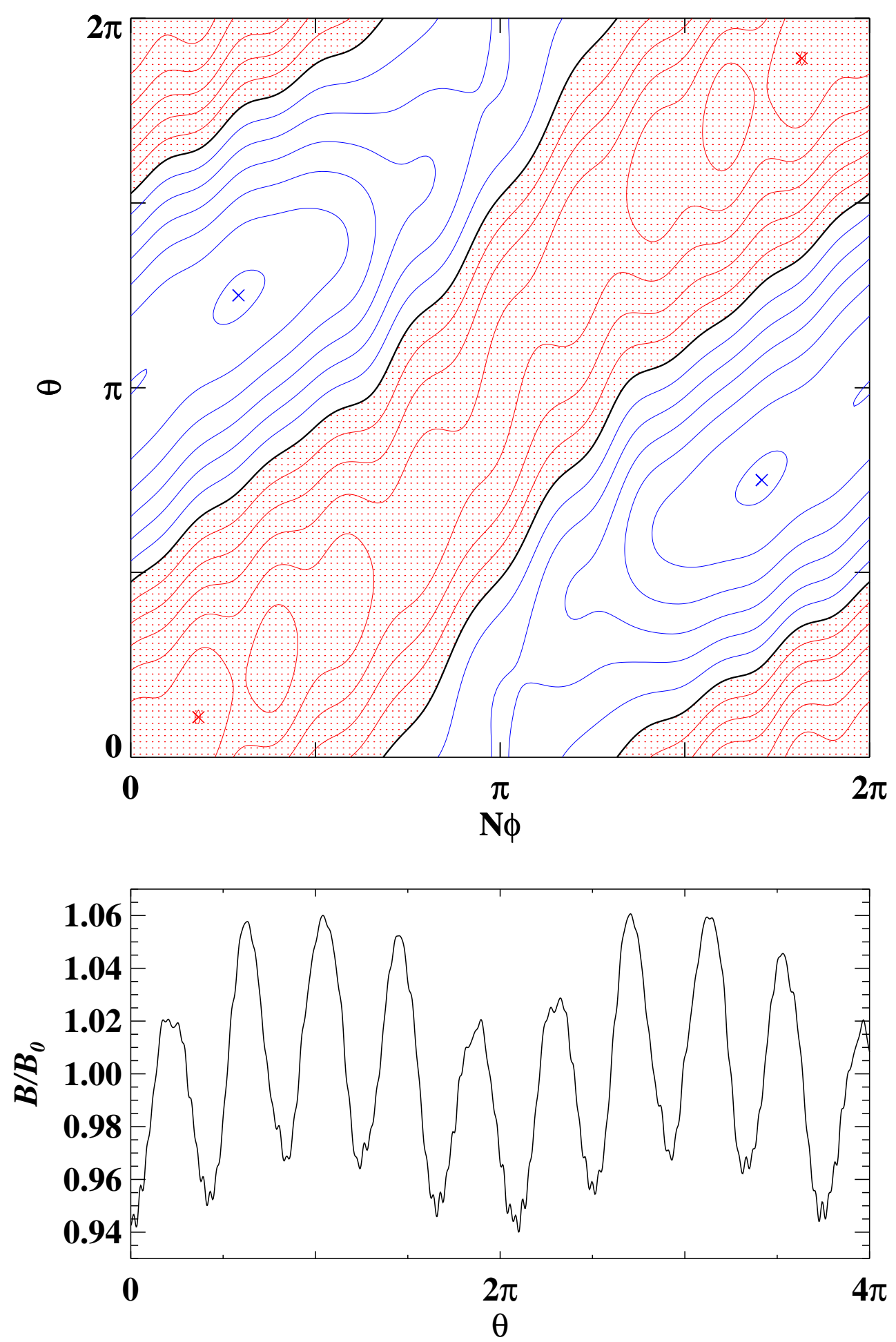

Figure 9a. Contours of constant magnetic field strength for one field period of W7-X (with $N=5$ ) are shown for the $\rho=0.5$ flux surface of the low-mirror vacuum configuration which has $t=0.8623$. Contours have been plotted following the same scheme employed in Figure 1. In the lower frame, the magnitude of $B / B_{0}$ along the field line passing through $\phi=0$ and $\theta=0$ is plotted over two poloidal circuits of the torus. 

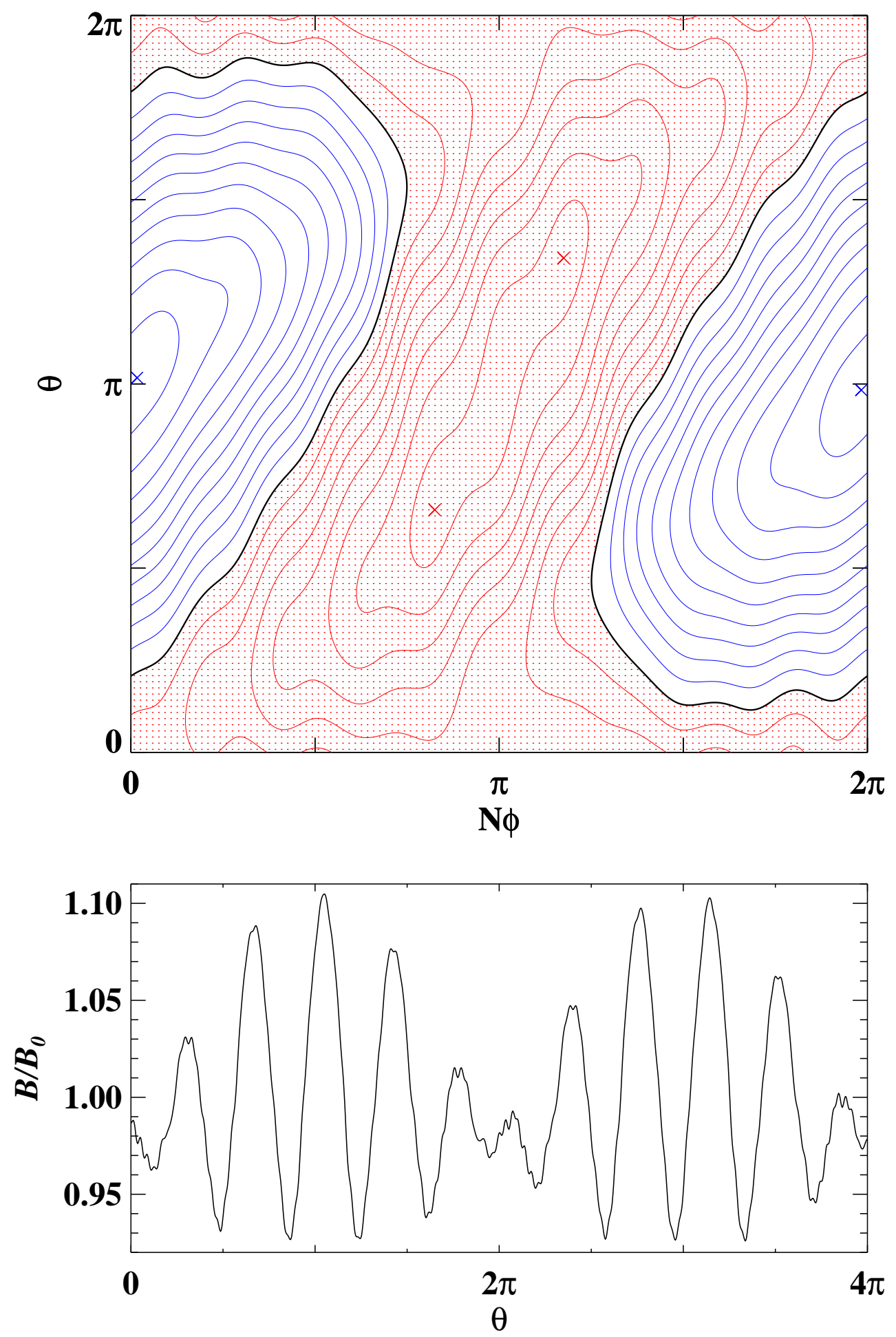

Figure 9b. Contours of constant magnetic field strength for one field period of W7-X (with $N=5$ ) are shown for the $\rho=0.5$ flux surface of the standard vacuum configuration which has $t=0.8700$. Contours have been plotted following the same scheme employed in Figure 1. In the lower frame, the magnitude of $B / B_{0}$ along the field line passing through $\phi=0$ and $\theta=0$ is plotted over two poloidal circuits of the torus. 

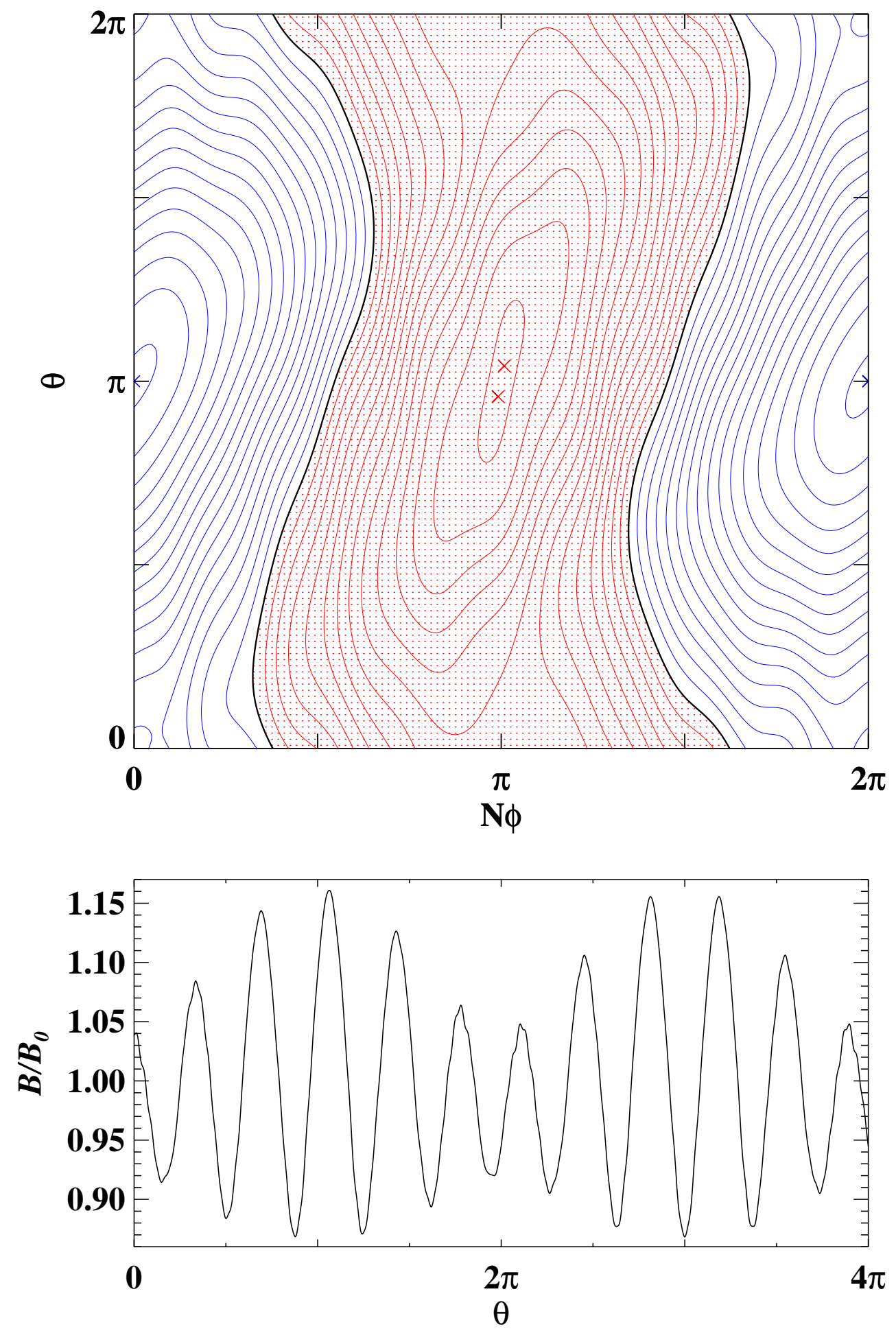

Figure 9c. Contours of constant magnetic field strength for one field period of W7-X (with $N=5$ ) are shown for the $\rho=0.5$ flux surface of the high-mirror vacuum configuration which has $t=0.8823$. Contours have been plotted following the same scheme employed in Figure 1. In the lower frame, the magnitude of $B / B_{0}$ along the field line passing through $\phi=0$ and $\theta=0$ is plotted over two poloidal circuits of the torus. 


\begin{tabular}{|l|c|c|c|}
\hline$N$ & CHS & LHD Standard & LHD Inward-Shifted \\
$R_{0}(\mathrm{~m})$ & 8 & 10 & 10 \\
$a(\mathrm{~m})$ & 0.9210 & 3.7481 & 3.6024 \\
\hline$r(\mathrm{~m})$ & 0.1905 & 0.5585 & 0.5400 \\
$t$ & 0.0953 & 0.2793 & 0.2700 \\
$\left|b_{m, n}\right|>0.01$ & -0.4067 & 0.4542 & 0.4692 \\
& $b_{1,0}=-0.09447$ & $b_{1,0}=-0.07053$ & $b_{1,0}=-0.05927$ \\
& $b_{2,-1}=0.06058$ & $b_{2,1}=0.05067$ & $b_{2,1}=0.05267$ \\
& $b_{0,1}=-0.01156$ & $b_{1,1}=-0.01476$ & $b_{1,1}=-0.04956$ \\
No. of $\left|b_{m, n}\right|>10^{-3}$ & 13 & & $b_{0,1}=0.01045$ \\
No. of $\left|b_{m, n}\right|>10^{-4}$ & 32 & 5 & 9 \\
\hline
\end{tabular}

\begin{tabular}{|l|c|c|c|}
\hline$N$ & W7-AS & TJ-II & HSX \\
$R_{0}(\mathrm{~m})$ & 5 & 4 & 4 \\
$a(\mathrm{~m})$ & 2.0183 & 1.5106 & 1.2375 \\
\hline$r(\mathrm{~m})$ & 0.1776 & 0.1776 & 0.1195 \\
$t$ & 0.0888 & 0.0888 & 0.0598 \\
$\left|b_{m, n}\right|>0.01$ & 0.3525 & -1.4759 & 1.0537 \\
& $b_{1,0}=-0.03074$ & $b_{1,-1}=-0.06269$ & $b_{1,1}=-0.07039$ \\
& $b_{1,-1}=0.02050$ & $b_{1,0}=-0.05893$ & \\
& $b_{0,2}=-0.01462$ & $b_{1,-2}=0.04030$ & \\
No. of $\left|b_{m, n}\right|>10^{-3}$ & & $b_{0,8}=-0.01375$ & 10 \\
No. of $\left|b_{m, n}\right|>10^{-4}$ & 48 & $b_{0,1}=0.01159$ & 61 \\
\hline
\end{tabular}

Table I. Magnetic field data obtained from VMEC equilibria for the twelve configurations used in the ICNTS benchmarking. In the upper portion of each column appears the name of the configuration and its field period number, $N$, along with the major radius, $R_{0}$, and radius of the last closed flux surface, $a$, for the equilibrium considered. Data for the magnetic flux surface $r$ are given in the lower portion of each column including the value of rotational transform, $t$, all Boozer harmonics of $B / B_{0}$ with magnitudes exceeding one percent on this flux surface as well as the number of harmonics for which $\left|b_{m, n}\right|$ exceeds the thresholds $10^{-3}$ and $10^{-4}$. 


\begin{tabular}{|c|c|c|c|}
\hline $\begin{array}{l}N \\
R_{0}(\mathrm{~m}) \\
a(\mathrm{~m})\end{array}$ & $\begin{array}{c}\text { NCSX } \\
3 \\
1.4654 \\
0.3230\end{array}$ & $\begin{array}{c}\text { QPS } \\
2 \\
0.9173 \\
0.3463\end{array}$ & $\begin{array}{c}\text { QIPC } \\
6 \\
11.846 \\
1.0270\end{array}$ \\
\hline$r(\mathrm{~m})$ & 0.1615 & 0.1732 & 0.5135 \\
\hline$t$ & 0.4942 & 0.1985 & 0.9137 \\
\hline$\left|b_{m, n}\right|>0.01$ & $b_{1,0}=-0.06848$ & $\begin{array}{c}b_{0,1}=0.16243 \\
b_{0,2}=-0.09310 \\
b_{1,0}=-0.05516 \\
b_{1,1}=-0.04576 \\
b_{1,2}=0.02929 \\
b_{1,-1}=0.01643 \\
b_{2,0}=-0.01294\end{array}$ & $\begin{array}{c}b_{0,1}=-0.22879 \\
b_{0,2}=0.05894 \\
b_{1,1}=0.04338 \\
b_{1,-1}=-0.02321 \\
b_{0,3}=-0.02078 \\
b_{1,-2}=0.01999 \\
b_{1,0}=-0.01689 \\
b_{1,2}=-0.01680 \\
b_{1,-3}=-0.01397 \\
b_{0,4}=0.01005\end{array}$ \\
\hline No. of $\left|b_{m, n}\right|>10^{-3}$ & 15 & 40 & 27 \\
\hline No. of $\left|b_{m, n}\right|>10^{-4}$ & 60 & 134 & 83 \\
\hline
\end{tabular}

\begin{tabular}{|l|c|c|c|}
\hline$N$ & W7-X Standard & W7-X Low-Mirror & W7-X High-Mirror \\
$R_{0}(\mathrm{~m})$ & 5 & 5 & 5 \\
$a(\mathrm{~m})$ & 5.5267 & 5.5276 & 5.5248 \\
\hline$r(\mathrm{~m})$ & 0.5109 & 0.5135 & 0.5122 \\
$t$ & 0.2555 & 0.2568 & 0.2561 \\
$\left|b_{m, n}\right|>0.01$ & 0.8700 & 0.8623 & 0.8823 \\
& $b_{0,1}=0.04645$ & $b_{1,1}=-0.04335$ & $b_{0,1}=0.10243$ \\
& $b_{1,1}=-0.04351$ & $b_{1,0}=-0.01794$ & $b_{1,1}=-0.04384$ \\
No. of $\left|b_{m, n}\right|>10^{-3}$ & $b_{1,0}=-0.01902$ & & $b_{1,0}=-0.02051$ \\
No. of $\left|b_{m, n}\right|>10^{-4}$ & 14 & 12 & 15 \\
& 50 & 56 & 54 \\
\hline
\end{tabular}


criteria relevant to good plasma performance. Additionally, the dimensions of W7-X $\left(R_{0}=\right.$ $5.5 \mathrm{~m}, a>0.5 \mathrm{~m}$ ) along with its heating and support systems were chosen to enable the high-performance, steady-state discharges necessary to assess the potential of such a device for attractive reactor operation. For experimental flexibility the W7-X coil system is capable of numerous magnetic configurations, among which are examples of the various neoclassical optimisation strategies discussed here, with the exception of quasi-symmetry (as small bootstrap current was an optimisation criterion for W7-X). At its simplest, this strategy involves nothing more than the large average elongation of the W7-X flux surfaces, which lies in the range $4.5<\kappa<7.0$ depending on the configuration. The magnetic field for such a case is exemplified by the W7-X low-mirror vacuum configuration plotted in Figure 9a, which is obtained by choosing the coil currents so as to zero the toroidal-mirror term (the $b_{0,1}$ component of $B$ in Boozer coordinates) on the magnetic axis. Unlike W7-AS, deeply trapped particles "see" only a rather small variation of $B$ in this field and thus experience the full benefit of the large reduction in average toroidal curvature, leading to a significant decrease in the neoclassical radial transport. Further improvements are possible, however, by introducing a modest toroidal mirror into $B$ so as to simultaneously profit from large elongation and strong drift optimisation, as is done for the W7-X standard configuration shown in Figure $9 \mathrm{~b}$ (which has equal currents in all non-planar coils, resulting in $b_{0,1}=0.046$ on axis). By further increasing the toroidal mirror, W7-X has access to quasi-isodynamic equilibria at volume-averaged $\beta$ values in excess of $2 \%$ for which the diamagnetic effect modifies the radial dependence of the magnetic field strength so as to produce an average-minimum- $B$ configuration $[73,74]$. An example of this type is provided by the high-mirror W7-X (with $b_{0,1}=0.1$ on axis), depicted in Figure $9 \mathrm{c}$ for the vacuum case. The mono-energetic bootstrap current coefficients are small for all three W7-X configurations due to the approximate cancellation of the individual contributions attributable to the dominant toroidal and helical components of $B\left(b_{1,0}\right.$ and $b_{1,1}$, respectively). The residual portion of $D_{31}$ can be influenced considerably by varying the toroidal mirror component of $B$, and reaches its smallest asymptotic value for the high-mirror case.

Magnetic field data obtained from VMEC equilibria for the twelve configurations investigated within the ICNTS benchmarking are provided in Table I. The major radius of the equilibrium and the minor radius of its last closed flux surface (in terms of its flux-surface label) are given for the configuration in the upper portion of each column; the lower portion contains data specific to the single flux surfaces depicted in the corresponding figures of this section including the value of rotational transform, the individual Boozer harmonics of $B / B_{0}$ with magnitudes larger than one percent and the number of harmonics for which $\left|b_{m, n}\right|$ exceeds the values $10^{-3}$ and $10^{-4}$. These two thresholds are of relevance for the numerical tools which employ a spectral representation of the magnetic field strength as accurate determination of the mono-energetic transport coefficients typically requires that per mill harmonics of $B$ are accounted for while for some configurations it is even necessary to include all $\left|b_{m, n}\right|>10^{-4}$. 


\section{Benchmarking Results}

For later use in calculations of the neoclassical fluxes for a given magnetic field configuration of interest, it is convenient to precalculate and then store normalised mono-energetic transport coefficients as functions of the flux-surface label, (mono-energetic) collisionality, $\nu^{\star} \equiv$ $R_{0} \nu /(t v)$, and normalised $\mathbf{E}_{r} \times \mathbf{B}$ drift velocity, $v_{E}^{\star}=E_{r} /\left(v B_{0}\right)$, for which they have been determined. For the benchmarking results presented in this section, normalisations to the results for an axisymmetric tokamak with $B / B_{0}=1-\epsilon_{t} \cos \theta$ in the plateau $(p)$, banana $(b)$ and Pfirsch-Schlüter $(P S)$ regimes have been chosen:

$$
\begin{array}{rr}
D_{11}^{\star} \equiv \frac{D_{11}}{D_{11}^{p}}, & D_{11}^{p}=\frac{\pi}{4} \frac{v_{d}^{2} R_{0}}{v t} ; \\
D_{31}^{\star} \equiv \frac{D_{31}}{D_{31}^{b}}, & D_{31}^{b}=\frac{2}{3} \frac{v_{d} R_{0}}{t \epsilon_{t}}\left(1-f_{c}\right) ; \\
D_{33}^{\star} \equiv \frac{D_{33}}{D_{33}^{P S}}, & D_{33}^{P S}=\frac{v^{2}}{3 \nu} \frac{\left\langle B^{2}\right\rangle}{B_{0}^{2}} .
\end{array}
$$

In these equations, $f_{c}$ is the fraction of circulating (non-reflected) particles which for the largeaspect-ratio approximation used here is given by $f_{c}=1-1.46 \sqrt{\epsilon_{t}}$. For the axisymmetric tokamak with $\mathrm{B} \cdot \boldsymbol{\nabla} B=-\epsilon_{t}(\mathrm{~B} \times \boldsymbol{\nabla} B) \cdot \nabla r$, one can show that a single mono-energetic transport coefficient is sufficient to describe all neoclassical effects [19] as the other two may be determined from

$$
\begin{gathered}
D_{11}=\frac{1}{t \epsilon_{t}} \frac{m}{q B_{0}} \frac{B_{0}^{2}}{\left\langle B^{2}\right\rangle} \nu D_{31}+\frac{8}{3}\left(\frac{v_{d} R_{0}}{v t}\right)^{2} \nu \\
D_{31}=\frac{1}{t \epsilon_{t}} \frac{m}{q B_{0}}\left(\frac{v^{2}}{3}-\frac{B_{0}^{2}}{\left\langle B^{2}\right\rangle} \nu D_{33}\right) .
\end{gathered}
$$

Using these expressions, it is straightforward to determine the collisional and collisionless asymptotes of the three normalised mono-energetic transport coefficients for the large-aspectratio, axisymmetric tokamak

$$
\begin{aligned}
D_{11}^{\star}\left(\nu^{\star} \rightarrow \infty\right)=\frac{32}{3 \pi} \nu^{\star}, & D_{11}^{\star}\left(\nu^{\star} \rightarrow 0\right) \approx 2.5 \frac{\nu^{\star}}{\epsilon_{t}^{3 / 2}}, \\
D_{31}^{\star}\left(\nu^{\star} \rightarrow \infty\right)=0, & D_{31}^{\star}\left(\nu^{\star} \rightarrow 0\right)=1, \\
D_{33}^{\star}\left(\nu^{\star} \rightarrow \infty\right)=1, & D_{33}^{\star}\left(\nu^{\star} \rightarrow 0\right)=f_{c} .
\end{aligned}
$$



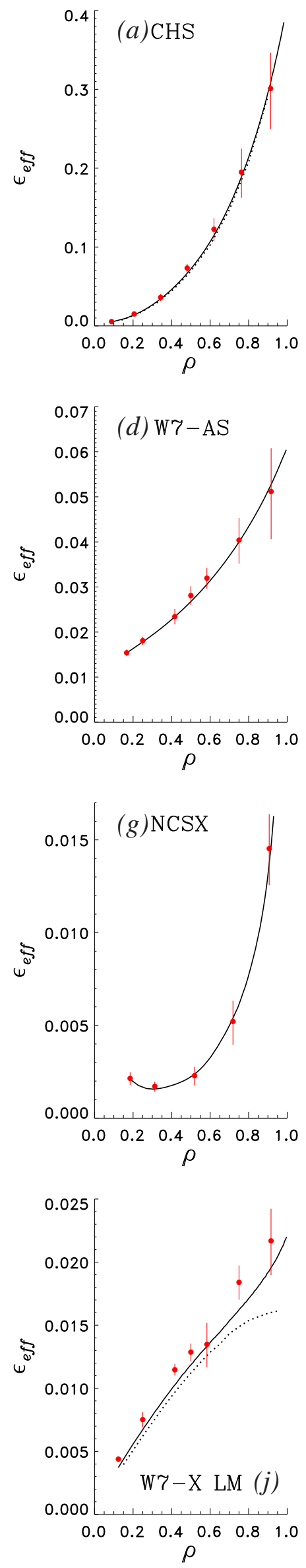
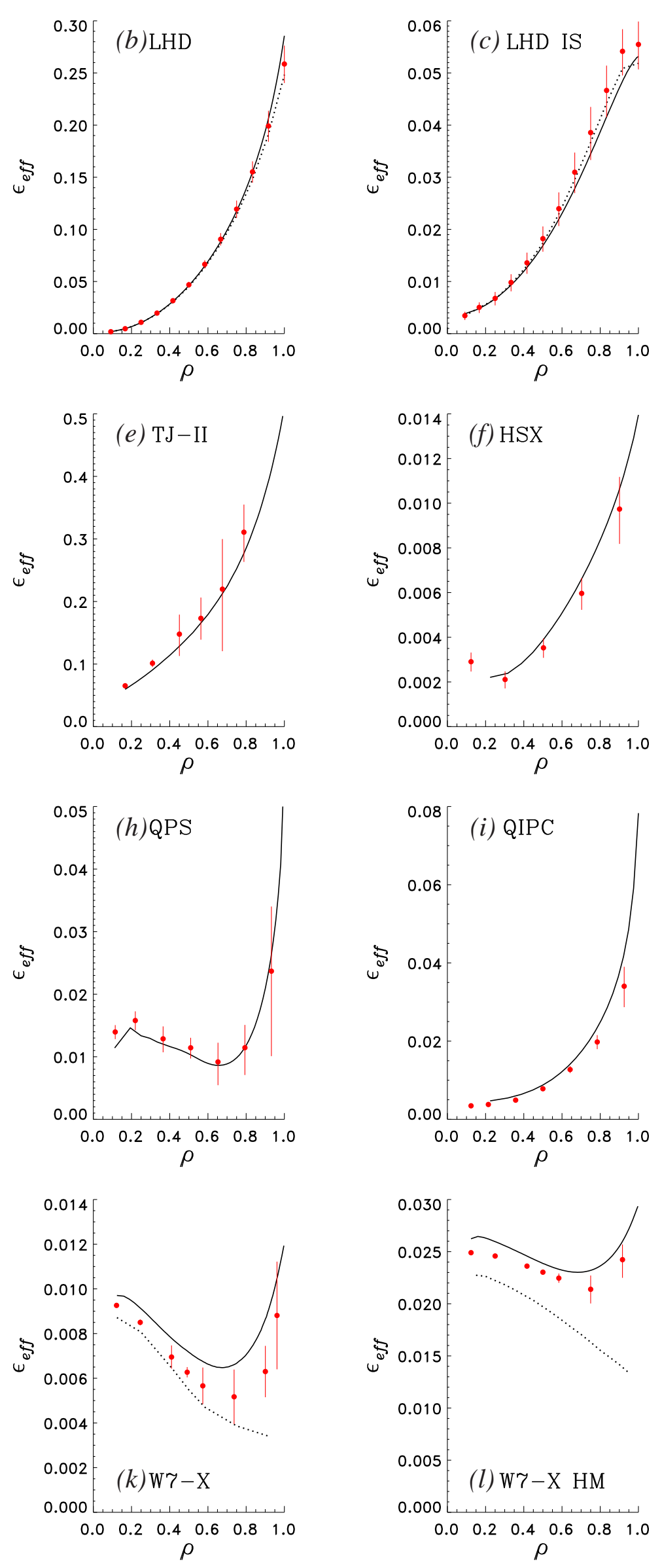
Figure 10. Radial profiles of the effective helical ripple for $1 / \nu$ transport are shown for the magnetic configurations of: (a) CHS, (b) LHD standard, (c) LHD inward-shifted, (d) W7-AS, (e) TJ-II, (f) HSX, (g) NCSX, (h) QPS, (i) QIPC, (j) W7-X low-mirror, ( $k$ ) W7-X standard and (l) W7-X high-mirror. Results from NEO for $\nu^{\star} \rightarrow 0$ are shown by the continuous (black) curves; estimates from DKES are depicted by the (red) data points with upper and lower bounds on the results indicated by "error bars"; where applicable, analytic results obtained assuming a multiple-helicity model for $B$ are plotted by the (black) dotted curve.

Also commonly used as a figure of merit to indicate the level of $1 / \nu$ transport in stellarators is the so-called effective helical ripple, $\epsilon_{\text {eff }}[75,76]$, which is determined from

$$
D_{11}^{\star}=\left(\frac{4}{3 \pi}\right)^{2} \frac{\left(2 \epsilon_{e f f}\right)^{3 / 2}}{\nu^{\star}}
$$

for the limiting case $\nu^{\star} \rightarrow 0$ and $E_{r}=0$ (a slightly different definition of effective helical ripple has been used previously to quantify results from NEO [8] but in the current paper $\epsilon_{e f f}$ has always been calculated from the formula given above). As the name implies, this quantity is defined such that $\epsilon_{e f f}=\epsilon_{h}$ for the characterisation of $1 / \nu$ transport in the simple model field of eq. (5). Improvement of the neoclassical confinement in stellarators is achieved most "economically" by choosing a magnetic field topology with small $\epsilon_{\text {eff }}$, making minimisation of this quantity one of the most common goals of stellarator optimisation efforts. Field-line integration assuming $\nu^{\star} \rightarrow 0$ is the most efficient numerical approach for determining $\epsilon_{e f f}$ and full radial profiles thereof, calculated by NEO, are given by the continuous curves in Figure 10 for each of the twelve configurations considered here. For comparison, DKES estimates of the effective helical ripple are also plotted for several flux surfaces, taken from calculations at the smallest values of $\nu^{\star}$ for which numerical convergence can still be claimed (the DKES data points are the average of the upper and lower variational bounds on the result with these bounds indicated in the figure by "error bars"). For the magnetic fields which can be approximated to a reasonable degree of accuracy using the multiple-helicity model [37], profiles of $\epsilon_{\text {eff }}$ obtained from analytic theory [38] are also shown by dotted lines.

A truly omnigenous device has, by definition, no $1 / \nu$ regime and hence $\epsilon_{\text {eff }}=0$. Given this observation, one may also interpret $\epsilon_{e f f}$ as a means of quantifying the departure from this ideal, condensing all information regarding the number of offending orbits and their deviations from the flux surface into a single value. For CHS, the standard configuration of LHD, W7-AS and NCSX the radial profiles of $\epsilon_{e f f}$ correspond very closely with the average depth of the local ripples, increasing quadratically to large values at the outer radii of the two heliotrons while remaining small for the quasi-axisymmetric NCSX as would be expected for this "stellarator 
approximation" to a rippled tokamak. The results for the inward-shifted LHD illustrate the benefits of drift optimisation in heliotrons as $\epsilon_{e f f}$ is reduced by a factor of five in comparison with the standard configuration although the number of localised particles in these two cases is nearly identical. It is also possible to significantly increase the effective helical ripple in LHD by an outward shift of the plasma column [77] but such a case was not included in the ICNTS, leaving only TJ-II to provide an example of such a "drift-amplified" configuration. QIPC exhibits the largest disparity between $\epsilon_{e f f}$ and the average depth of local ripples as the latter exceeds the former by a factor of fifty for the inner flux surfaces.

With respect to benchmarking, the NEO results for $\epsilon_{\text {eff }}$ fall within the bounds determined by DKES in the great majority of cases, the maximum discrepancy leading to an uncertainty in $D_{11}$ of less than $20 \%$. Analytic theory is of comparable accuracy only for the three heliotron configurations; in the remaining cases it underestimates the $1 / \nu$ transport as a high degree of optimisation can be noticeably diminished by even small departures from the multiple-helicity model for $B$ used by the theory. The accuracy of the $\epsilon_{\text {eff }}$ values determined by the other numerical methods used in the course of the ICNTS benchmarking will become apparent from the $E_{r}=0$ portion of the results for $D_{11}^{\star}\left(\nu^{\star}, v_{E}^{\star}\right)$ presented in the next subsection.

\subsection{The Mono-Energetic Radial Transport Coefficient $-D_{11}^{\star}$}

In the following, benchmarking results for the normalised mono-energetic radial transport coefficient, $D_{11}^{\star}$, will be presented as functions of $\nu^{\star}$ and $v_{E}^{\star}$ for a single flux surface of selected configurations. One surface is deemed sufficient for the current purposes as the quality of agreement between different numerical approaches is seldom a function of the flux surface label for which calculations have been performed. (The small number of cases where this is not true will be pointed out where they arise.)

As a first example, results from DKES, NEO-2, GSRAKE and five Monte Carlo codes are compared in Figure 11 for the standard configuration of LHD. Six different values of $v_{E}^{\star}$ are considered and indicated by the colour code given in the figure caption. For reference purposes, the dotted curve depicts the results for the "equivalent" axisymmetric tokamak with magnetic field strength $B / B_{0}=1+b_{1,0} \cos \theta$ and values of $r, R_{0}, t$, and $b_{1,0}$ identical with those of LHD. The highest collisionalities plotted here are well into the Pfirsch-Schlüter regime where pitchangle scattering is so frequent that the concept of trapped particles becomes meaningless and accurate estimates of $D_{11}^{\star}$ for both stellarators and tokamaks need account only for the toroidal nature of these devices, leading to the analytic result [78]

$$
D_{11}^{\star}=\frac{32}{3 \pi} \frac{\nu^{\star}}{\kappa}\left[1+\left(\frac{3 v_{E}^{\star} \nu^{\star}}{t \epsilon_{t}}\right)^{2}\right]^{-1} .
$$

Traditionally, poloidal $\mathbf{E}_{r} \times \mathbf{B}$ precession is ignored when solving the drift kinetic equation in 


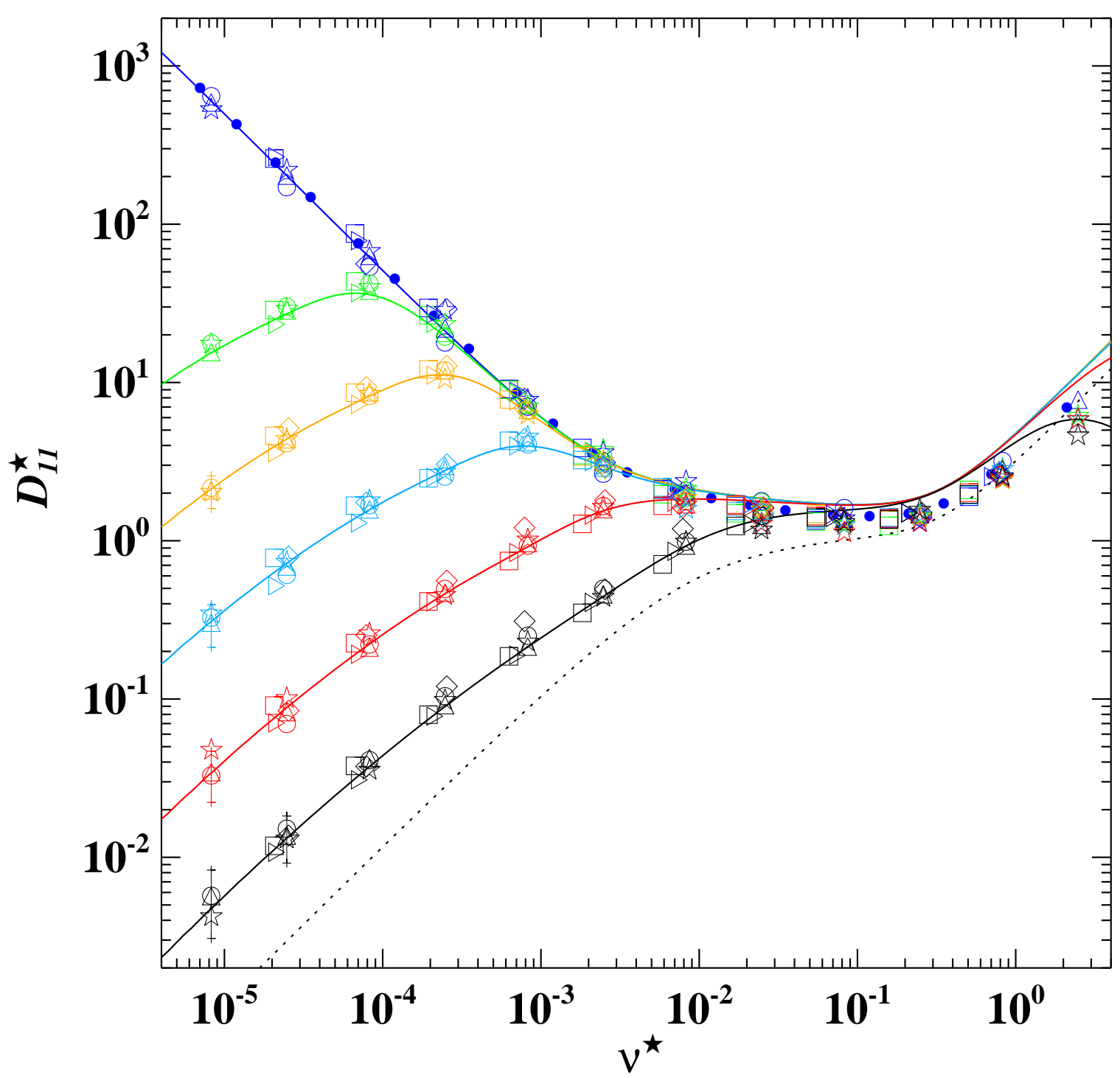

Figure 11. Normalised mono-energetic radial transport coefficient as a function of collisionality for $v_{E}^{\star}=E_{r} /\left(v B_{0}\right)=3 \times 10^{-3}, 1 \times 10^{-3}, 3 \times 10^{-4}, 1 \times 10^{-4}, 3 \times 10^{-5}$ and zero for the LHD standard configuration at $\rho=0.5$. Numerical results from GSRAKE are depicted as continuous curves, those from NEO-2 as small filled-in circles $(\bullet)$ and from DKES as triangles $(\triangle)$ with upper and lower variational bounds indicated when these lie outside the symbol. Results from five different Monte Carlo codes are plotted as circles $(\bigcirc)$ [12], squares $(\square)$ [10], diamonds $(\diamond)$ [11], stars $(\diamond)$ [13] and right-pointing triangles $(\triangleright)[15]$. For comparison, results for the equivalent axisymmetric tokamak $(r=0.2793 \mathrm{~m}$, $\left.R_{0}=3.7481 \mathrm{~m}, t=0.4542, b_{1,0}=-0.07053\right)$ are shown by the dotted line for $E_{r}=0$. 
the Pfirsch-Schlüter regime but, as can be seen, it becomes relevant once the product of radial electric field and collisionality is sufficiently large. Moving to lower $\nu^{\star}$, the Pfirsch-Schlüter regime gives way to a clearly recognisable plateau regime with $D_{11}^{\star}$ values somewhat larger than those of the equivalent axisymmetric tokamak due to the small additional contributions attributable to the helical components of $B$ [79]. At yet lower collisionality textbook examples of stellarator $\operatorname{lmfp}$ transport are on display with $1 / \nu$ transport evident until it is suppressed by the poloidal $\mathbf{E}_{r} \times \mathbf{B}$ precession of localised particles once $\nu^{\star} / v_{E}^{\star}$ falls below a certain threshold. With regard to the accuracy of the numerical approaches, upper and lower variational bounds on the DKES results are plotted only when these extend outside the symbol (triangle) used to mark their average. For the Monte Carlo results, ensembles of more than 1000 simulation particles were commonly employed for the ICNTS benchmarking although in some runs at the lowest values of collisionality this number was reduced to as few as 250 . Even in the latter case, the relative standard error never exceeds $15 \%$, corresponding roughly to the vertical extent of the symbols used in the figures so that the $95 \%$ confidence interval for these results is at most a factor of two larger (but not shown explicitly to avoid cluttering the figure). The numerical convergence for NEO-2 and GSRAKE may be considered exact for all results shown in this plot; GSRAKE overestimates the transport at high collisionality where the ripple average (which is performed over collisionless particle trajectories) leads to an improper weighting of the drifts responsible for transport at these values of $\nu^{\star}$.

Benchmarking results for the inward-shifted LHD are presented in Figure 12 to illustrate the effects which strong drift optimisation has on $D_{11}^{\star}$ in classical heliotrons. Noteworthy in this regard is that the benefits of the optimisation are not confined to the $\operatorname{lm} f p$ regime (i.e. collisionalities satisfying $\nu^{\star}<(4 / 3 \pi)^{2}\left(2 \epsilon_{\text {eff }}\right)^{3 / 2} \kappa$, for which the mono-energetic $1 / \nu$ transport coefficient exceeds the plateau value of the equivalent axisymmetric tokamak) but also leads to modest reductions of $D_{11}^{\star}$ at higher collisionality as well, as a comparison with the results for the LHD standard configuration will show. Within the $\operatorname{lmfp}$ regime it will be noted that small non-zero values of the radial electric field (e.g. $v_{E}^{\star}=3 \times 10^{-5}$ and $10^{-4}$ ) now produce a much more gradual "roll-over" of the transport coefficients than previously, displaying rather broad ranges of collisionality over which $D_{11}^{\star}$ is only weakly dependent on $\nu^{\star}$. To understand this consequence of strong drift optimisation, one must recall that both collisions and poloidal precession can set constraints on the radial excursions of localised particles in the stellarator $\operatorname{lmfp}$ regime, with the relative importance of these constraints given by the ratio $\nu_{c} / \Omega_{E}$, where $\nu_{c}=\nu / \mathcal{F}^{2}$ is the collisional detrapping frequency, with $\mathcal{F}$ the fraction of phase space through which a particle must be displaced if collisions are to remove it from a local ripple, and $\Omega_{E}=$ $E_{r} /\left(r B_{0}\right)$ is the $\mathbf{E}_{r} \times \mathbf{B}$ precession frequency. Particles with $\nu_{c} / \Omega_{E}>1$ participate in $1 / \nu$ transport with a characteristic time step proportional to $\nu_{c}^{-1}$, making drift optimisation of deeply trapped particles highly desirable as they must be scattered through a fraction of phase space corresponding to the full depth of the local ripple and therefore have the smallest $\nu_{c}$. At the 


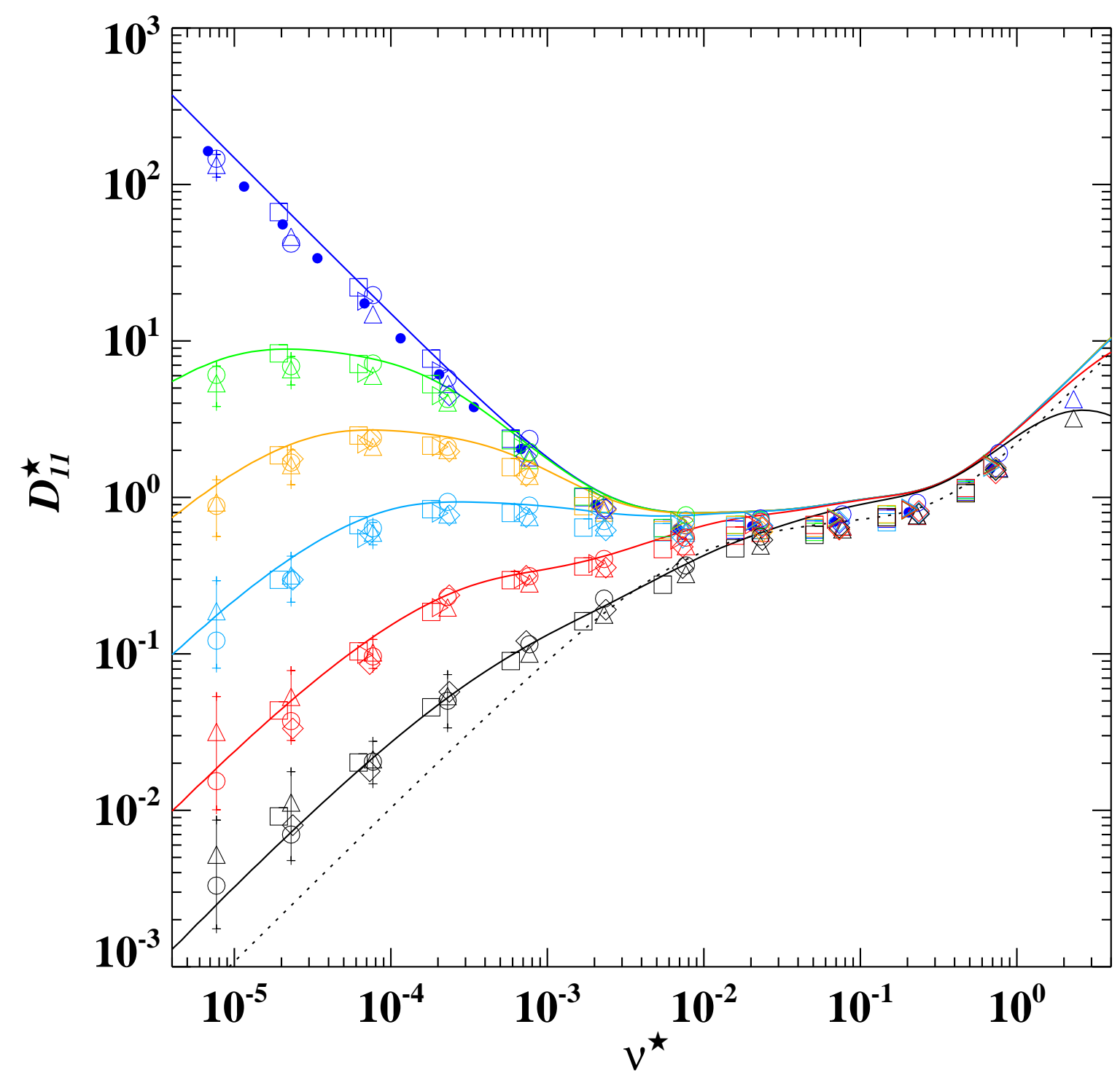

Figure 12. Normalised mono-energetic radial transport coefficient as a function of the collisionality for $v_{E}^{\star}=3 \times 10^{-3}, 1 \times 10^{-3}, 3 \times 10^{-4}, 1 \times 10^{-4}, 3 \times 10^{-5}$ and zero for the LHD inward-shifted configuration at $\rho=0.5$. Numerical results from GSRAKE are depicted as continuous curves, those from NEO-2 as small filled-in circles $(\bullet)$ and from DKES as triangles $(\triangle)$ with upper and lower variational bounds indicated when these lie outside the symbol. Monte Carlo results are plotted as circles $(\bigcirc)$ [12], squares $(\square)[10]$, diamonds $(\diamond)[11]$ and right-pointing triangles $(\triangleright)$ [15]. For comparison, results for the equivalent axisymmetric tokamak $\left(r=0.27 \mathrm{~m}, R_{0}=3.6024 \mathrm{~m}, t=0.4692, b_{1,0}=-0.0593\right)$ are shown by the dotted line for $E_{r}=0$. 
same time, however, as the collisionality decreases deeply trapped particles become the first to have their radial displacements limited by $\Omega_{E}$, signifying the end of the $1 / \nu$ regime even though collisional removal of shallowly trapped particles from the local ripples will persist due to their smaller values of $\mathcal{F}$. Thus, for a stellarator magnetic field without drift optimisation, the beneficial effects of the $\mathrm{E}_{r} \times \mathrm{B}$ precession first act on those particles making the largest contribution to the transport so that only a small additional decrease in collisionality is necessary to reach the roll-over point of the $D_{11}^{\star}$ results. By design, however, deeply trapped particles contribute little to the transport in strongly drift-optimised stellarators so that $\nu_{c} / \Omega_{E} \approx 1$ is required for even shallowly trapped particles before the roll-over can occur, pushing this point to considerably smaller values of $\nu^{\star}[76]$.

One might also expect the influence of the radial electric field on $D_{11}^{\star}$ to be modified by more complicated magnetic field topologies, especially those in which deep secondary minima in $B$ are present. This was not observed for either W7-AS or TJ-II, however, the two most likely candidates among the ICNTS configurations. Instead, the dependence of the radial transport coefficients on $E_{r}$ was found to be (qualitatively) identical to that of a classical stellarator as illustrated by the results for TJ-II plotted in Figure 13. The comparatively poor convergence of the DKES results for this device at low collisionality is due to its very broad Fourier spectrum of $B$ in Boozer coordinates; at outer radii this problem is further exacerbated and convergence is no longer satisfactory for $\nu^{\star}<10^{-4}$.

Among the configurations optimised for small neoclassical losses, benchmarking results for $D_{11}^{\star}$ are presented here for HSX (Figure 14), NCSX (Figure 15), QIPC (Figure 16) and the standard configuration of W7-X (Figures 17 and 18). For comparison with the HSX results the dotted curve depicts the neoclassical transport in the equivalent helically symmetric field $B / B_{0}=1+b_{1,1} \cos (\theta-N \phi)$, obtained from a simple isomorphic transformation of the axisymmetric tokamak results [80]. In plotting the results, however, the normalisation remains the same in all figures, e.g. in the plateau regime one expects $D_{11}^{\star}=\left(b_{1,1} / \epsilon_{t}\right)^{2} t / t_{t}-N \mid \approx 0.75$ for HSX while for NCSX one has $D_{11}^{\star}=1 / \kappa \approx 0.36$. The approximation to quasi-helical symmetry is sufficiently good for HSX that the banana regime of its helically symmetric counterpart may be identified in the range of collisionalities satisfying $3 \times 10^{-3}<\nu^{\star}<$ 0.03 , although typical stellarator behaviour of the $D_{11}^{\star}$ results is evident at lower values of collisionality even though it is of a magnitude small compared to that of classical stellarators. The NCSX data exhibits similar properties except that the banana regime appears less distinctly as this regime first emerges at lower collisionality in the equivalent axisymmetric device.

Stellarators with predominant $b_{0, n}$ harmonics in their Boozer-coordinate representations of $B$ are known to exhibit significantly modified behaviour of $D_{11}^{\star}$ over the range of collision frequencies in which the plateau regime would otherwise be expected to appear [81]. QIPC offers an excellent example of such behaviour as reference to the results for the equivalent axisymmetric tokamak (dotted curve in Figure 16) clearly illustrates. For $\nu^{\star}<5 \times 10^{-3}$, 


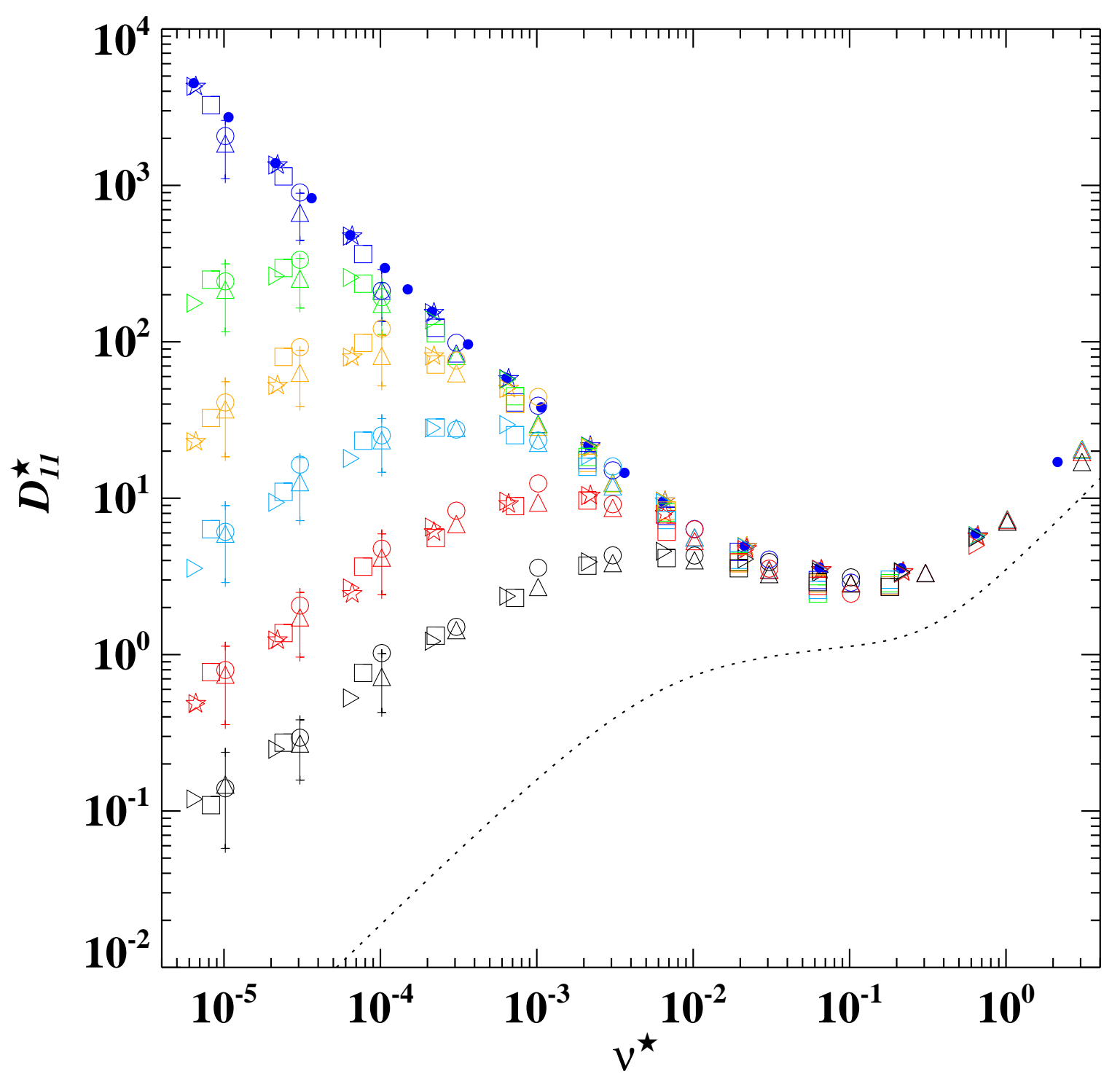

Figure 13. Normalised mono-energetic radial transport coefficient as a function of collisionality for $v_{E}^{\star}=3 \times 10^{-3}, 1 \times 10^{-3}, 3 \times 10^{-4}, 1 \times 10^{-4}, 3 \times 10^{-5}$ and zero for TJ-II at $\rho=0.46$. Numerical results from NEO-2 are depicted as small filled-in circles $(\bullet)$ and those from DKES as triangles $(\triangle)$ with upper and lower variational bounds indicated when these lie outside the symbol. Monte Carlo results

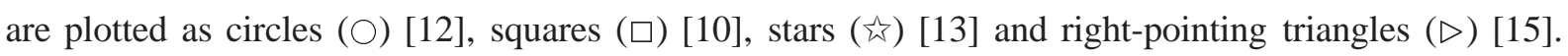
For comparison, results for the equivalent axisymmetric tokamak $\left(r=0.0815 \mathrm{~m}, R_{0}=1.5106 \mathrm{~m}\right.$, $\left.t=1.4753, b_{1,0}=-0.0533\right)$ are shown by the dotted line for $E_{r}=0$. 


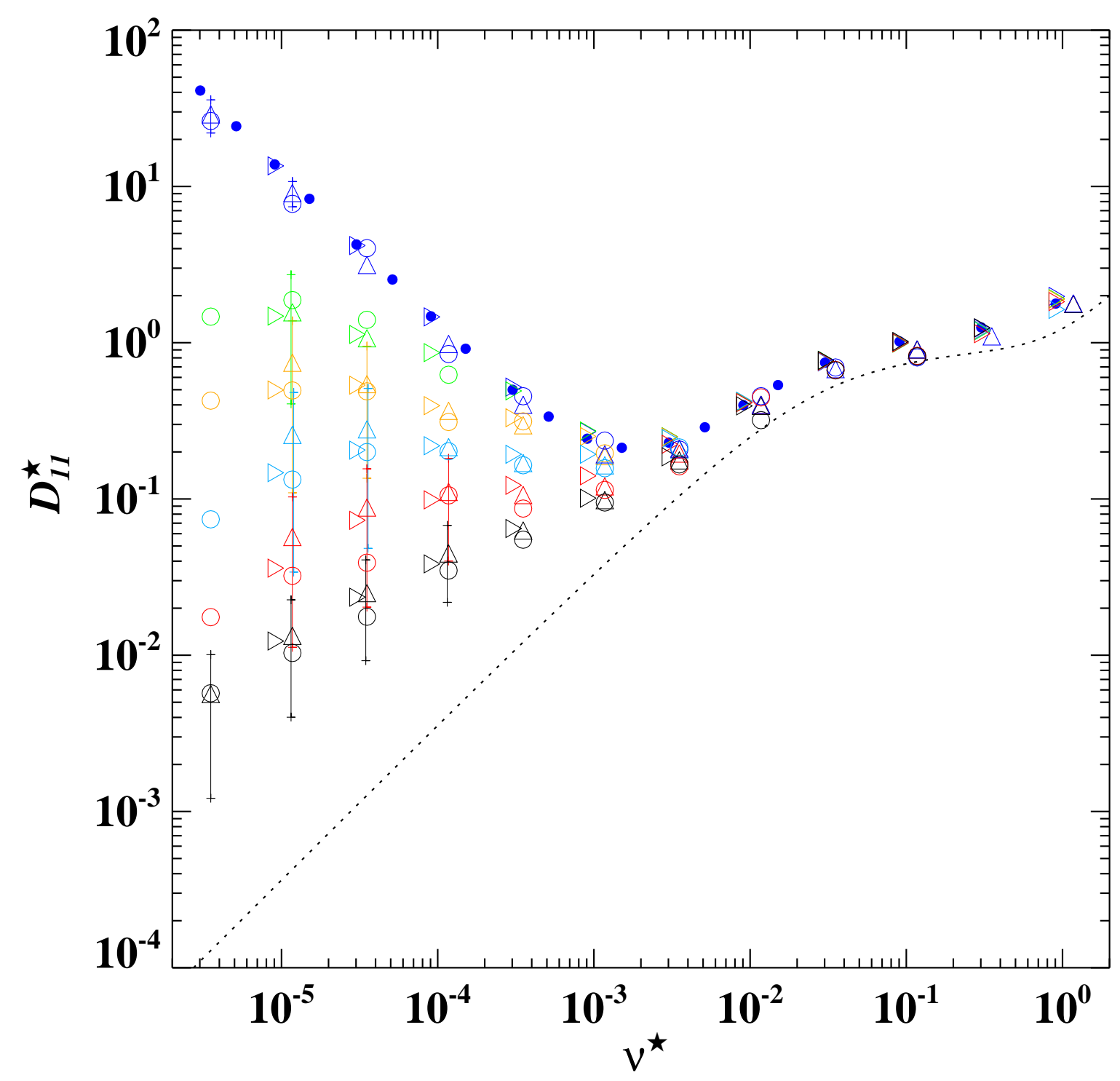

Figure 14. Normalised mono-energetic radial transport coefficient as a function of collisionality for $v_{E}^{\star}=3 \times 10^{-3}, 1 \times 10^{-3}, 3 \times 10^{-4}, 1 \times 10^{-4}, 3 \times 10^{-5}$ and zero for HSX at $\rho=0.5$. Numerical results from NEO-2 are depicted as small filled-in circles $(\bullet)$ and those from DKES as triangles $(\triangle)$ with upper and lower variational bounds indicated when these lie outside the symbol. Monte Carlo results are plotted as circles $(\bigcirc)$ [12] and right-pointing triangles $(\triangleright)$ [15]. For comparison, results for the equivalent helical symmetry $\left(r=0.0598 \mathrm{~m}, R_{0}=1.2375 \mathrm{~m}, t=1.0537, N=4, b_{1,1}=-0.07039\right)$ are shown by the dotted line for $E_{r}=0$. 


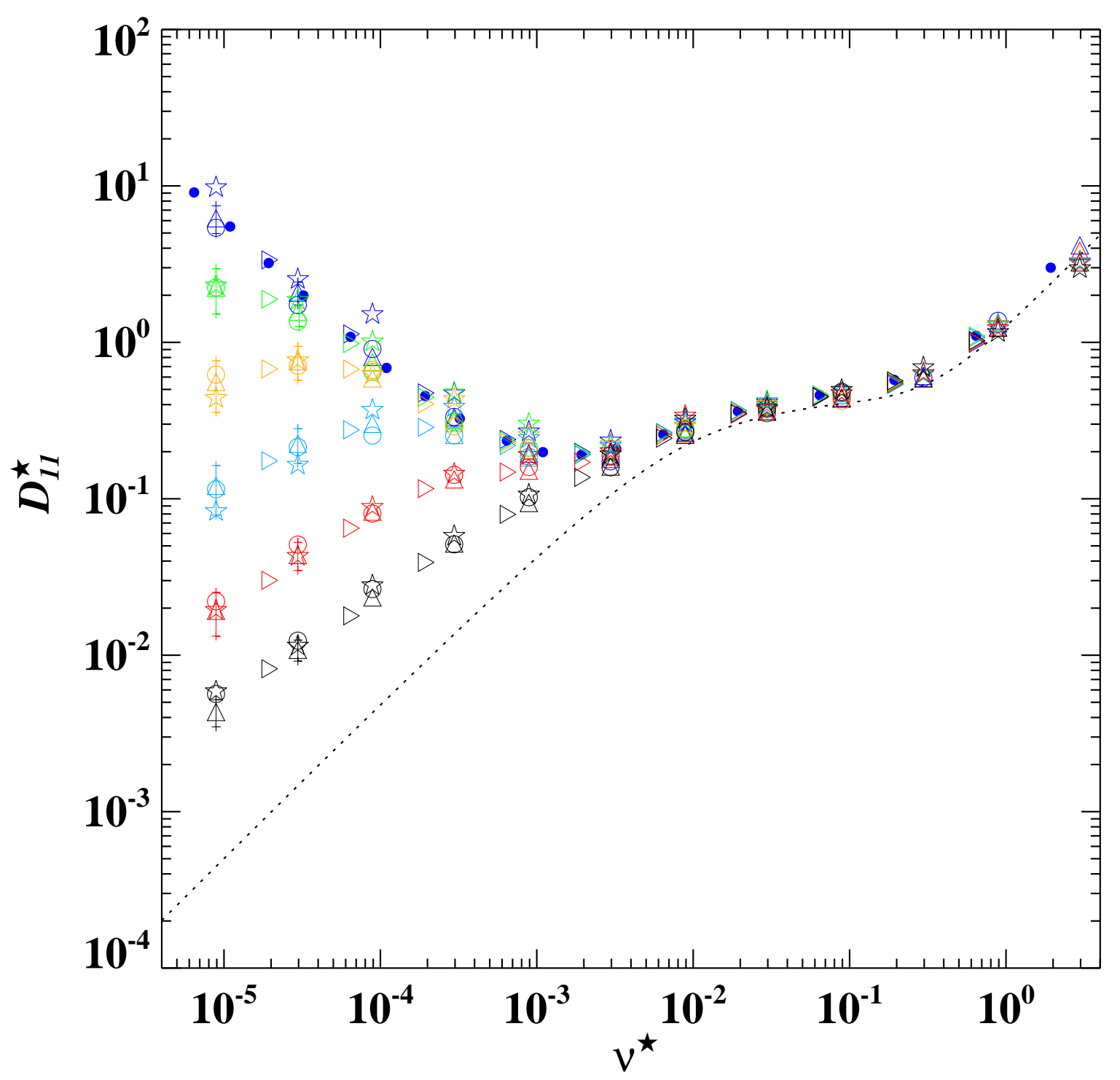

Figure 15. Normalised mono-energetic radial transport coefficient as a function of collisionality for $v_{E}^{\star}=3 \times 10^{-3}, 1 \times 10^{-3}, 3 \times 10^{-4}, 1 \times 10^{-4}, 3 \times 10^{-5}$ and zero for NCSX at $\rho=0.5$. Numerical results from NEO-2 are depicted as small filled-in circles $(\bullet)$ and those from DKES as triangles $(\triangle)$ with upper and lower variational bounds indicated when these lie outside the symbol. Monte Carlo results are plotted as circles $(\bigcirc)$ [12], stars $($ 放) [13] and right-pointing triangles $(\triangleright)$ [15]. For comparison, results for the equivalent axisymmetric tokamak $\left(r=0.1615 \mathrm{~m}, R_{0}=1.4654 \mathrm{~m}, t=0.4942, b_{1,0}=-0.06848\right)$ are shown by the dotted line for $E_{r}=0$. 


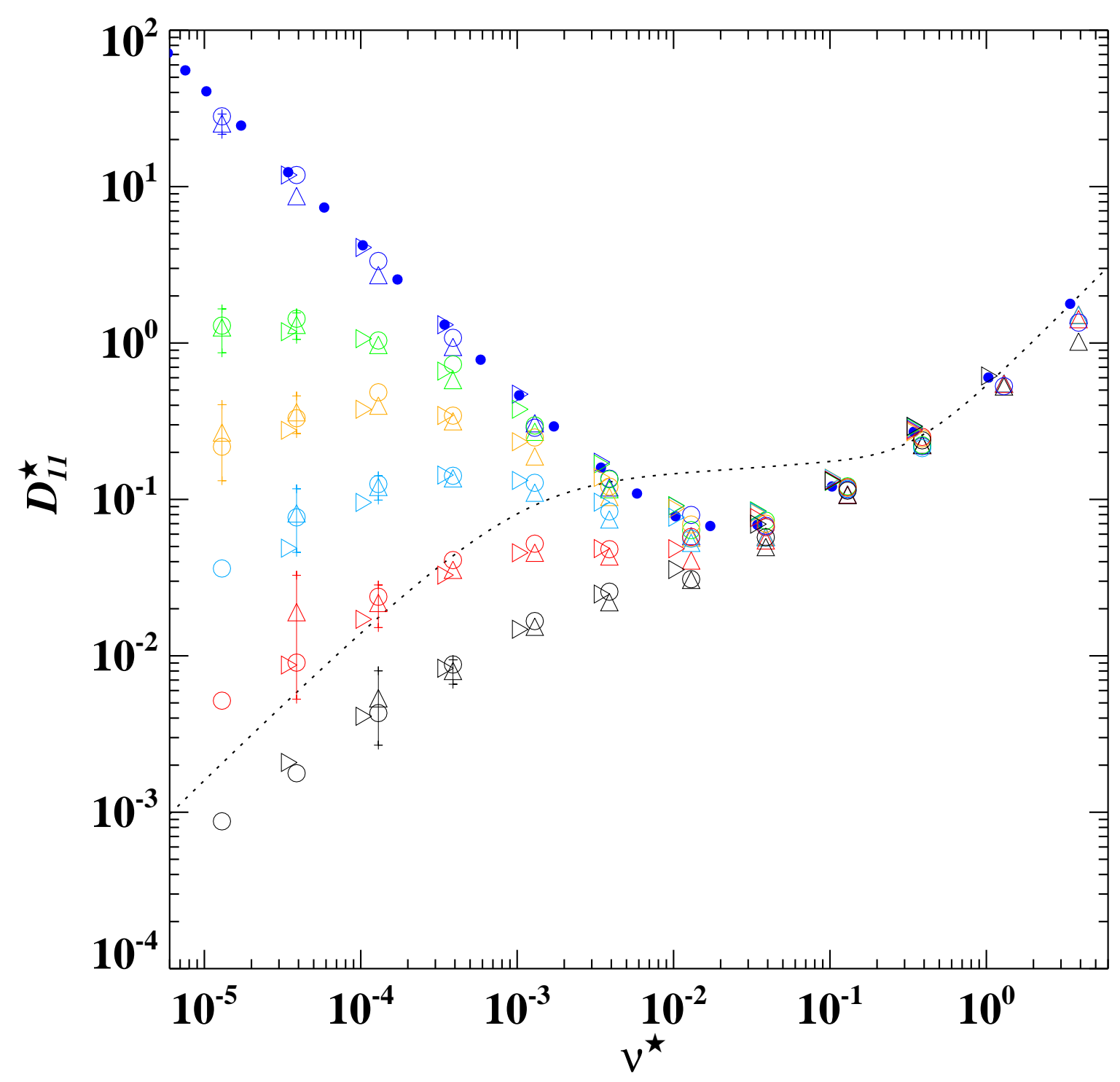

Figure 16. Normalised mono-energetic radial transport coefficient as a function of collisionality for $v_{E}^{\star}=3 \times 10^{-3}, 1 \times 10^{-3}, 3 \times 10^{-4}, 1 \times 10^{-4}, 3 \times 10^{-5}$ and zero for QIPC at $\rho=0.5$. Numerical results from NEO-2 are depicted as small filled-in circles $(\bullet)$ and those from DKES as triangles $(\triangle)$ with upper and lower variational bounds indicated when these lie outside the symbol. Monte Carlo results are plotted as circles $(\bigcirc)$ [12] and right-pointing triangles $(\triangleright)$ [15]. For comparison, results for the equivalent axisymmetric tokamak $\left(r=0.5135 \mathrm{~m}, R_{0}=11.846 \mathrm{~m}, t=0.9137, b_{1,0}=-0.01689\right)$ are shown by the dotted line for $E_{r}=0$. 


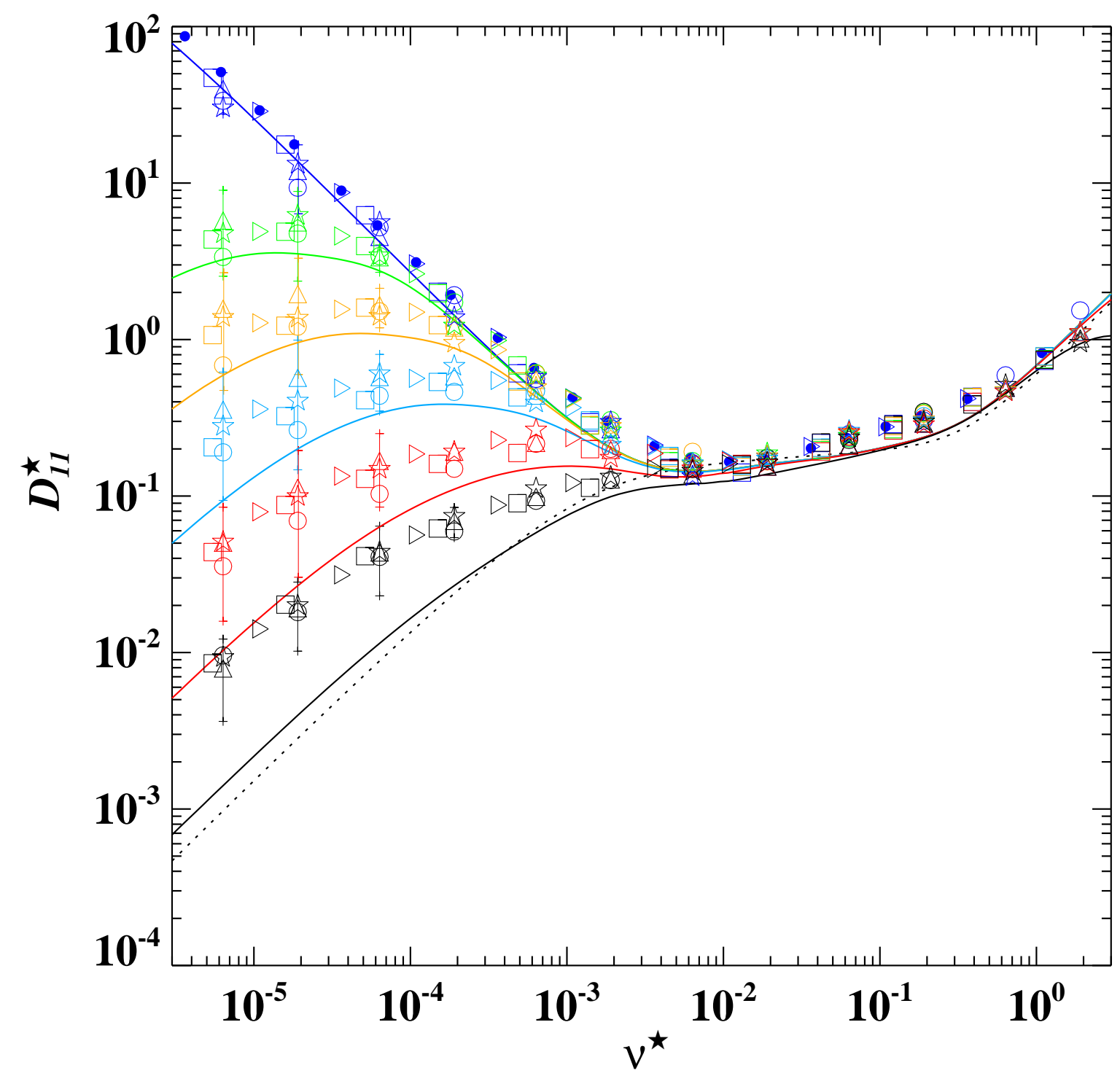

Figure 17. Normalised mono-energetic radial transport coefficient as a function of collisionality for $v_{E}^{\star}=3 \times 10^{-3}, 1 \times 10^{-3}, 3 \times 10^{-4}, 1 \times 10^{-4}, 3 \times 10^{-5}$ and zero for the W7-X standard configuration at $\rho=0.5$. Numerical results from GSRAKE are depicted as continuous curves, those from NEO-2 as small filled-in circles $(\bullet)$ and from DKES as triangles $(\triangle)$ with upper and lower variational bounds indicated when these lie outside the symbol. Results from four different Monte Carlo codes are plotted as circles $(\bigcirc)$ [12], squares $(\square)$ [10], stars (支) [13] and right-pointing triangles $(\triangleright)$ [15]. For comparison, results for the equivalent axisymmetric tokamak $\left(r=0.2555 \mathrm{~m}, R_{0}=5.5267 \mathrm{~m}, t=0.870, b_{1,0}=-0.01902\right)$ are shown by the dotted line for $E_{r}=0$. 


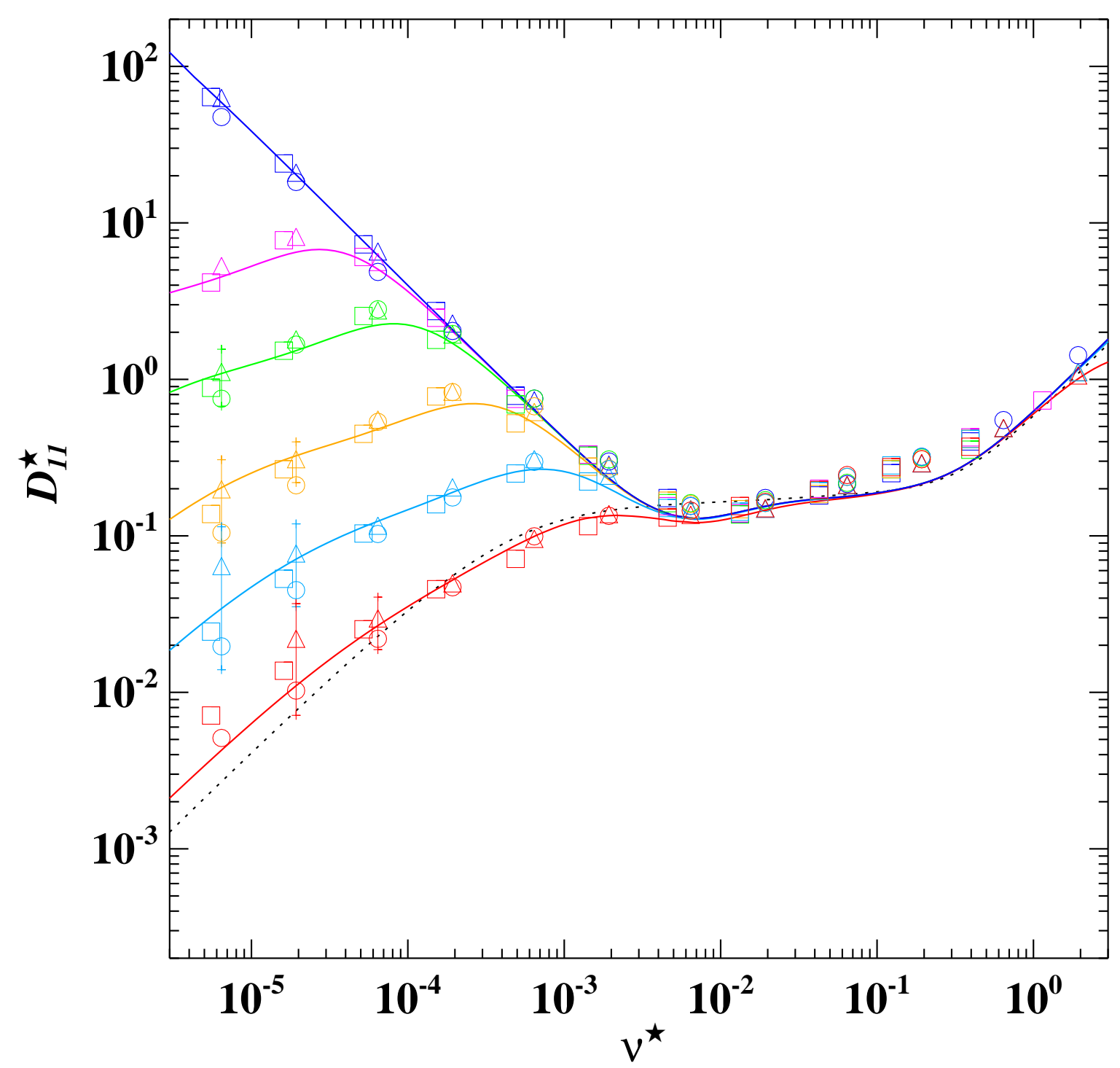

Figure 18. Normalised mono-energetic radial transport coefficient as a function of collisionality for $v_{E}^{\star}=1 \times 10^{-3}, 3 \times 10^{-4}, 1 \times 10^{-4}, 3 \times 10^{-5}, 1 \times 10^{-5}$ and zero for the standard configuration of W7-X at $\rho=0.25$. Numerical results from GSRAKE are depicted as continuous curves and those from DKES as triangles $(\triangle)$ with upper and lower variational bounds indicated when these lie outside the symbol. Results from Monte Carlo codes are plotted as circles ( $\bigcirc$ ) [12] and squares ( $\square$ ) [10]. For comparison, results for the equivalent axisymmetric tokamak $\left(r=0.1277 \mathrm{~m}, R_{0}=5.5267 \mathrm{~m}, t=0.8589, b_{1,0}=\right.$ $-0.00936)$ are shown by the dotted line for $E_{r}=0$. 
however, all the typical characteristics of $\operatorname{lmfp}$ radial transport in stellarators appear for this configuration as well. With regard to the numerical results, QIPC is noteworthy as the convergence of low-collisionality DKES runs shows unusually strong dependence on the magnitude of the radial electric field; for upper and lower variational bounds differing by considerably more than an order of magnitude the DKES results are considered unreliable and do not appear on the plots.

The use of GSRAKE in the ICNTS benchmarking activity has been restricted to the six configurations with magnetic field strengths which (at least for the majority of flux surfaces) can be accurately approximated using the multiple-helicity model [37,38]; these comprise CHS, the standard and inward-shifted LHD and the three W7-X configurations. For the heliotrons, GSRAKE results are consistently in good agreement with those of the other numerical methods but this is not always the case for W7-X as the example of Figure 17 demonstrates for the $\rho=0.5$ flux surface of the standard configuration. One notes particularly for these results that the accuracy of GSRAKE is satisfactory for $E_{r}=0$ but then steadily worsens as the value of the radial electric field is increased. This discrepancy diminishes quite rapidly, however, as the radius of the flux surface under consideration is decreased and is no longer evident for the $\rho=0.25$ results plotted in Figure 18. To summarise the full set of benchmarking results for W7-X, GSRAKE performs rather poorly for the standard configuration, accurately determining $D_{11}^{\star}\left(\nu^{\star}, v_{E}^{\star}\right)$ only for those flux surfaces with $\rho \leq 0.4$, while for the low-mirror and high-mirror configurations this "critical" radius moves closer to the plasma edge and thus to radii where the fairly poor description of $B$ provided by the multiple-helicity model noticeably influences the accuracy of GSRAKE calculations. Whether the model field also affects the accuracy of results for the standard configuration was investigated by performing a second set of DKES computations for the $\rho=0.5$ flux surface assuming the identical $B$ used by GSRAKE. Perceptible reductions of $D_{11}^{\star}$ for $\nu^{\star}<5 v_{E}^{\star}$ were indeed obtained with the model field but good agreement with GSRAKE results was only extended to the range of collisionalities $v_{E}^{\star} / 2<\nu^{\star}<5 v_{E}^{\star}$ with clear differences remaining at smaller $\nu^{\star}$. This could indicate that use of the ripple average is not always admissible for simplifying the full drift kinetic equation even if the structure of $B$ is elementary enough to pose no obvious difficulties but, on the other hand, it may simply point to shortcomings in the numerical implementation of GSRAKE. From the point of view of code benchmarking, however, it is sufficient to note that GSRAKE results must be taken with a grain of salt if corroborating data from Monte Carlo simulations and/or DKES is lacking.

\subsection{The Mono-Energetic Parallel Transport Coefficient $-D_{33}^{\star}$}

Calculation of the parallel transport coefficient in toroidal devices is historically associated with determining a plasma's electric conductivity. Unlike tokamaks, however, ohmic current 
plays at most a secondary role in establishing the poloidal component of the magnetic field in stellarators and this is reflected in the early scientific literature describing neoclassical transport in helical devices by a dearth of papers in which solutions of eq. (2) are presented. This situation has changed somewhat in recent years with the development of various momentum-correction techniques [19-21] which correct the flux-surface-averaged neoclassical flows so as to recover the conservation of parallel momentum which is violated by use of the Lorentz operator to describe collisions in the drift kinetic equation. Whether seeking the solution of a linear system of moment equations or the iterative solution of a generalised Spitzer problem, all momentumcorrection techniques require knowledge of $D_{33}$, and thus a means of solving eq. (2) becomes mandatory. Nevertheless, the choice of numerical tools for doing so remains rather limited; for the ICNTS benchmarking, results from DKES, NEO-2 and a $\delta f$ Monte Carlo code are compared in this subsection.

In Figure 19 the normalised mono-energetic parallel transport coefficient is plotted as a function of $\nu^{\star}$ and $v_{E}^{\star}$ for the standard configuration of LHD. Data sets for only three values of $E_{r}$ are considered sufficient here as the symmetry properties of the Vlasov operator cause one to expect that the antisymmetric portion of $\widehat{f}_{I}$ (and hence $D_{33}$ ) will be independent of the radial electric field as long as $v_{E}^{\star} \ll t \epsilon_{t}$, which is already an ordering assumption required to express the linearised drift kinetic equation in the mono-energetic form used here. As can be seen from the DKES and Monte Carlo results, this expectation is confirmed by these two numerical solutions of eq. (2); a very weak dependence of the DKES results on $E_{r}$ is observed for $v_{E}^{\star}=10^{-3}$ (especially for $5 \times 10^{-4}<\nu^{\star}<5 \times 10^{-2}$ ) but these changes in $D_{33}^{\star}$ are too small to be verified by Monte Carlo simulations given the statistical uncertainty inherent to this method of solution. As a consequence, the neglect of the $\mathbf{E} \times \mathbf{B}$ drift, which underlies the field-line-integration technique, represents no drawback to accurate calculations of the monoenergetic parallel transport coefficient and makes NEO-2 the most efficient numerical tool for this task.

For comparison, $D_{33}^{\star}\left(\nu^{\star}\right)$ for the equivalent axial and helical symmetries is given in Figure 19 by the dotted curves. At the highest collisionalities the results for both symmetries are identical as collisions are too frequent for details of the magnetic field structure to have any influence. The bounce frequency of localised particles in LHD is much higher than that of banana orbits in the equivalent tokamak, however, so that the collisionality at which trappedparticle effects begin to reduce $D_{33}^{\star}$ is correspondingly larger and coincides quite well with the $\nu^{\star}$ value at which the equivalent helical symmetry is first affected. In the collisionless limit the value of $D_{33}^{\star}$ asymptotically approaches the fraction of circulating particles (which never undergo reflection) which for an arbitrarily complex magnetic field is given by

$$
f_{c}=\frac{3}{4} \frac{\left\langle B^{2}\right\rangle}{B_{\max }^{2}} \int_{0}^{1} \mathrm{~d} \lambda \frac{\lambda}{\left\langle\sqrt{1-\lambda B / B_{\max }}\right\rangle}
$$




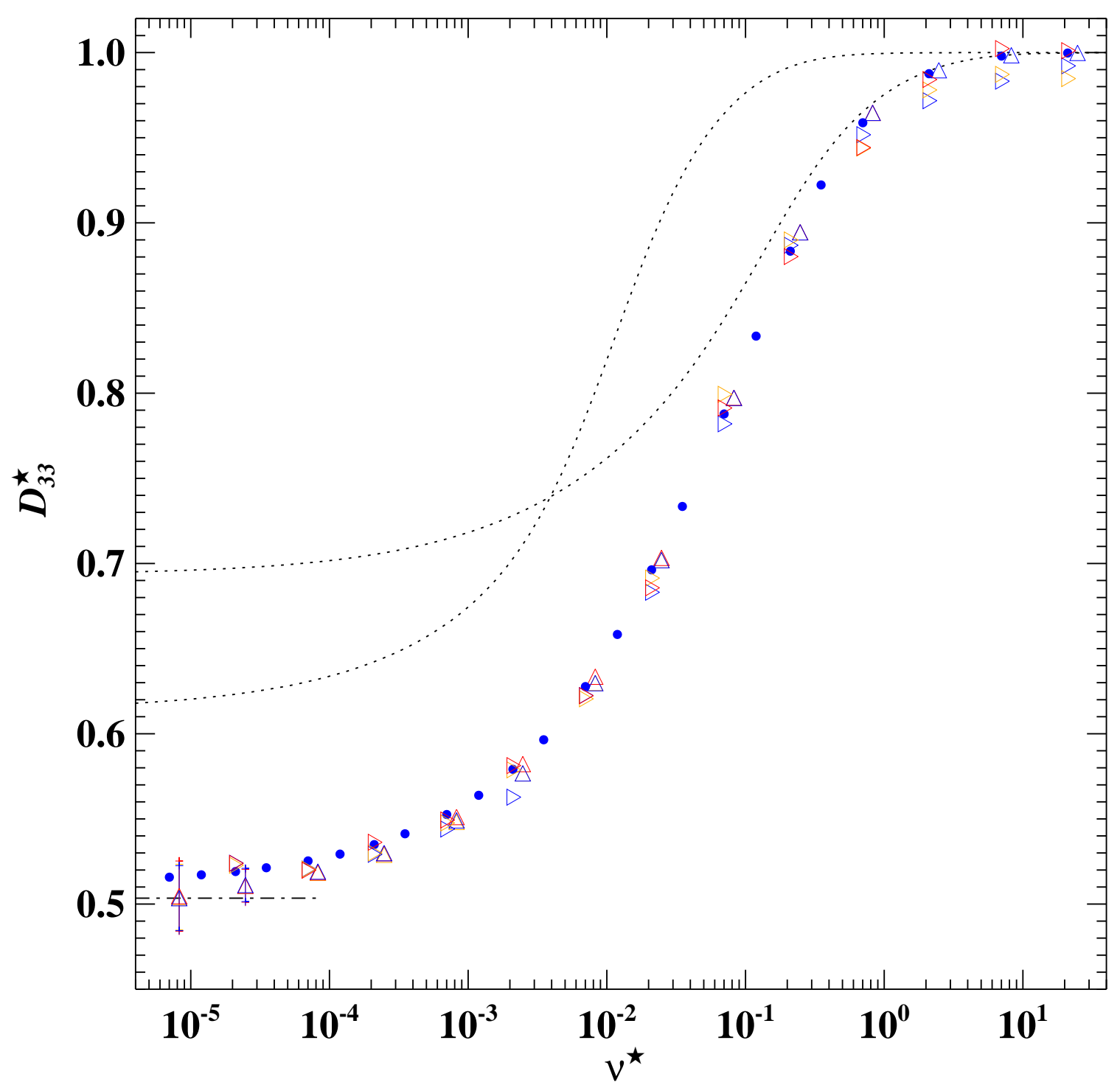

Figure 19. Normalised mono-energetic parallel transport coefficient as a function of collisionality for $v_{E}^{\star}=1 \times 10^{-3}, 1 \times 10^{-4}$ and zero for the LHD standard configuration at $\rho=0.5$. Numerical results from NEO-2 are depicted as small filled-in circles $(\bullet)$, those from DKES as triangles $(\triangle)$ with upper and lower variational bounds indicated when these lie outside the symbol and results from a Monte Carlo code $[15]$ are plotted as right-pointing triangles $(\triangleright)$. For comparison, results for the equivalent axisymmetric tokamak $\left(r=0.2793 \mathrm{~m}, R_{0}=3.7481 \mathrm{~m}, t=0.4542, b_{1,0}=-0.07053\right)$ and helical symmetry $\left(N=10, b_{2,1}=-0.05067\right)$ are shown by the dotted lines for $E_{r}=0$. The collisionless asymptote, the fraction of circulating particles, is indicated for the LHD standard configuration by the dot-dash line. 


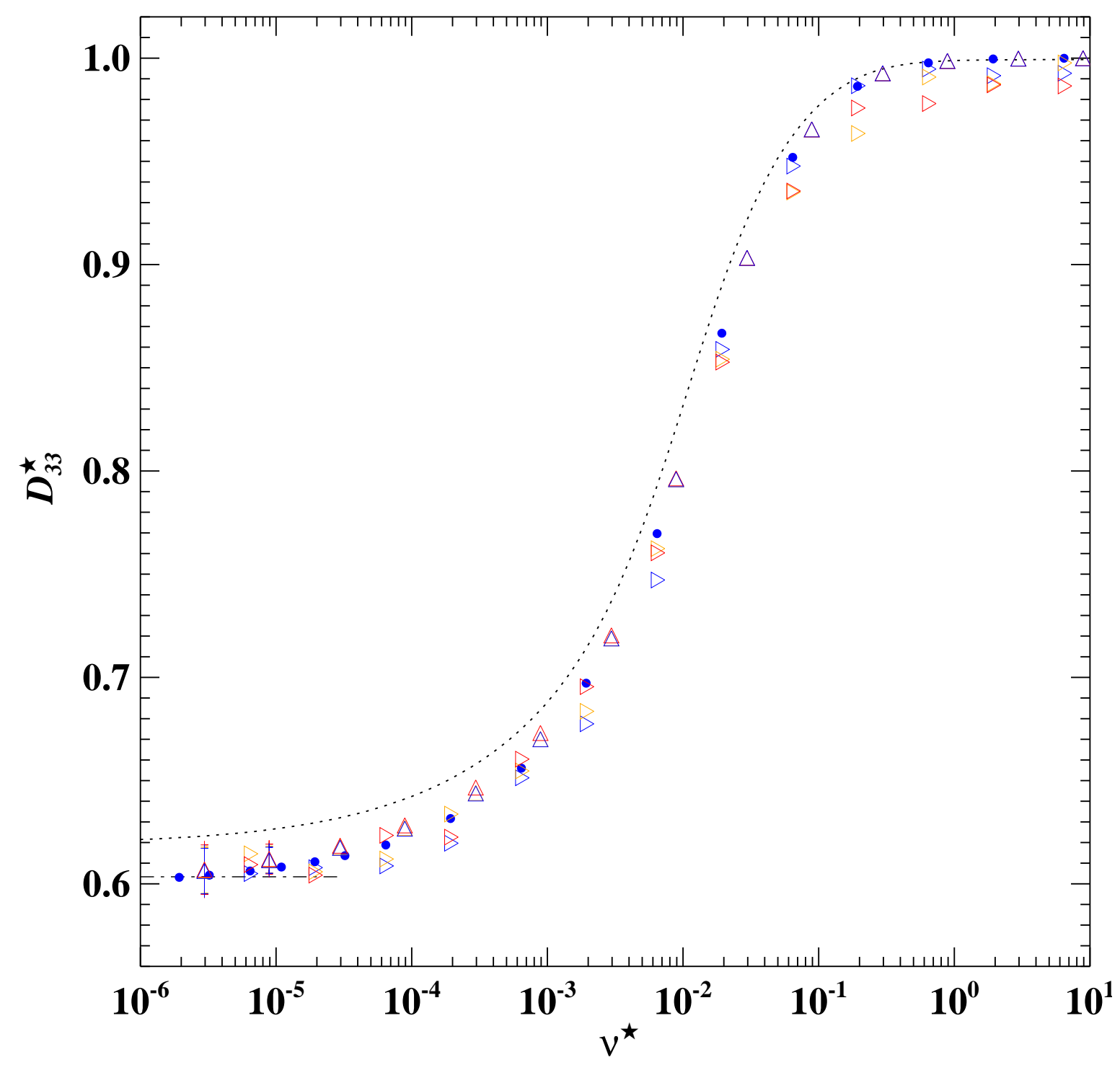

Figure 20. Normalised mono-energetic parallel transport coefficient as a function of collisionality for $v_{E}^{\star}=1 \times 10^{-3}, 1 \times 10^{-4}$ and zero for NCSX at $\rho=0.5$. Numerical results from NEO-2 are depicted as small filled-in circles $(\bullet)$, those from DKES as triangles $(\triangle)$ with upper and lower variational bounds indicated when these lie outside the symbol and results from a Monte Carlo code [15] are plotted as rightpointing triangles $(\triangleright)$. For comparison, results for the equivalent axisymmetric tokamak $(r=0.1615 \mathrm{~m}$, $\left.R_{0}=1.4654 \mathrm{~m}, t=0.4942, b_{1,0}=-0.06848\right)$ are shown by the dotted line for $E_{r}=0$. The collisionless asymptote, the fraction of circulating particles, is indicated for NCSX by the dot-dash line. 


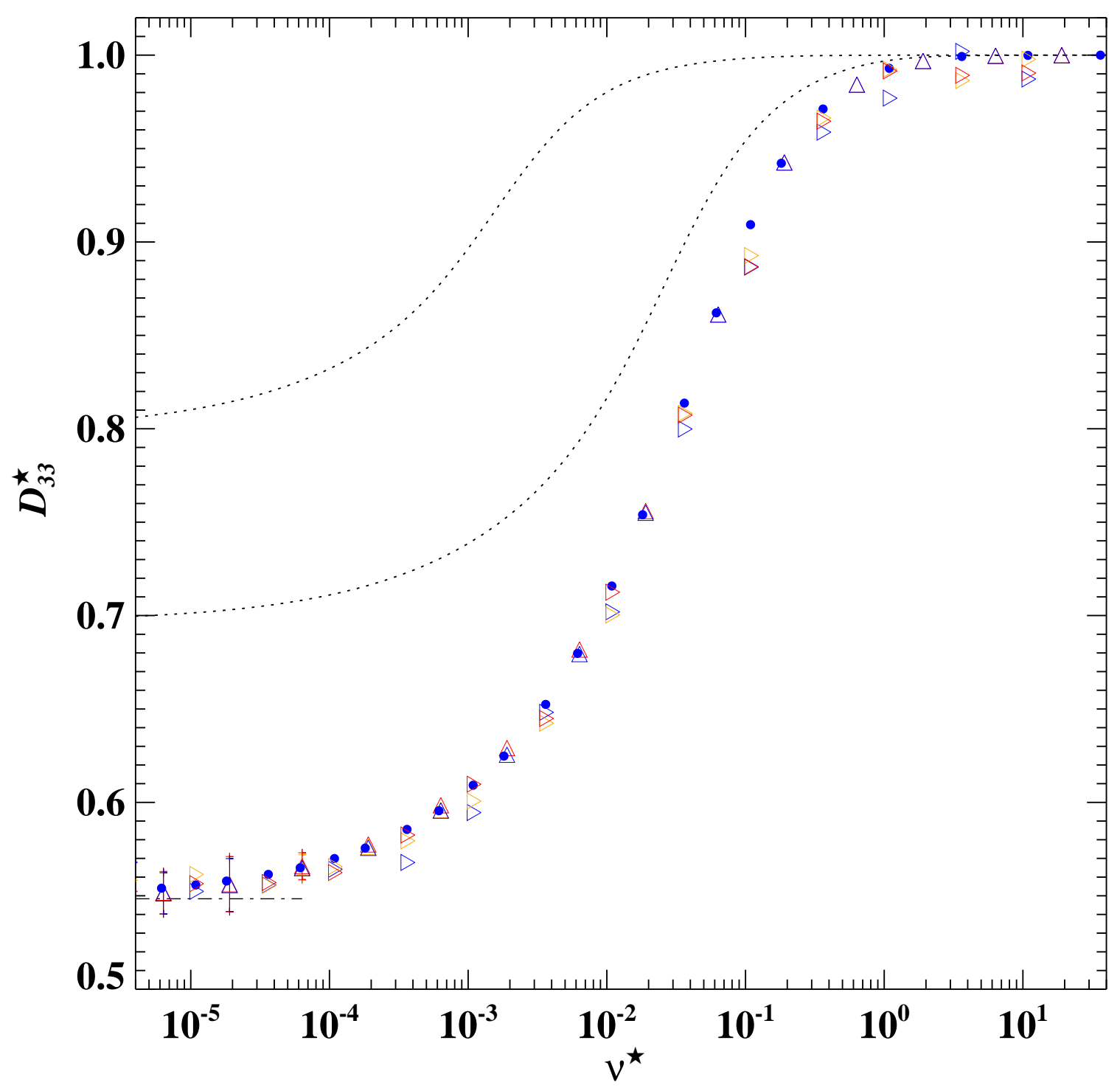

Figure 21. Normalised mono-energetic parallel transport coefficient as a function of collisionality for $v_{E}^{\star}=1 \times 10^{-3}, 1 \times 10^{-4}$ and zero for the W7-X standard configuration at $\rho=0.5$. Numerical results from NEO-2 are depicted as small filled-in circles $(\bullet)$, those from DKES as triangles $(\triangle)$ with upper and lower variational bounds indicated when these lie outside the symbol and results from a Monte Carlo code $[15]$ are plotted as right-pointing triangles $(\triangleright)$. For comparison, results for the equivalent axisymmetric tokamak $\left(r=0.2555 \mathrm{~m}, R_{0}=5.5267 \mathrm{~m}, t=0.870, b_{1,0}=-0.01902\right)$ and helical symmetry $\left(N=5, b_{1,1}=-0.04351\right)$ are shown by the dotted lines for $E_{r}=0$. The collisionless asymptote, the fraction of circulating particles, is indicated for the W7-X standard configuration by the dot-dash line. 
where $B_{\max }$ is the maximum value of $B$ on the flux surface. This quantity is easily determined by numerical integration and its value for LHD is indicated in the figure by the dot-dash line, providing an additional "analytic benchmark" for small- $\nu^{\star}$ results. The three sets of numerical results recover the expected values of $D_{33}^{\star}$ in both the collisional and collisionless limits and exhibit excellent agreement for all other values of collisionality as well, marking a successful benchmarking of the mono-energetic parallel transport coefficient for LHD.

The numerical calculations of $D_{33}^{\star}$ are in equally good agreement for the other ICNTS configurations and the qualitative behaviour of the results is uniformly in line with expectations so that it will suffice here to present only two further benchmarking examples. These are given in Figure 20 for NCSX and in Figure 21 for the standard configuration of W7-X. For NCSX, $D_{33}^{\star}\left(\nu^{\star}\right)$ departs only marginally from the curve for the equivalent tokamak in keeping with its approximation to quasi-symmetry; a similar conformity of the results for HSX and the equivalent helically symmetric configuration has also been obtained. For the remaining configurations, however, the $D_{33}^{\star}$ values bear little resemblance to any set of results obtained using a single-harmonic truncation of $B$, as the curves for W7-X serve to illustrate.

\subsection{The Mono-Energetic Bootstrap Current Coefficient $-D_{31}^{\star}$}

Benchmarking results are presented in this subsection for the last of the three mono-energetic transport coefficients, which may be said to characterise either the bootstrap current (when used in eq. (4) to determine $I_{3}$ ) or the Ware pinch (when calculating $I_{1}$ and $I_{2}$ ). Under common experimental conditions in stellarators the thermodynamic force $A_{3}$ is much too weak to produce an appreciable particle or energy pinch, however, so that the terminology mono-energetic bootstrap current coefficient is often applied interchangeably to both $D_{31}$ and $-D_{13}$. This convention is also followed here - all results are expressed in terms of the normalised mono-energetic bootstrap current coefficient, $D_{31}^{\star}$, regardless of whether they have been obtained from numerical solutions for the symmetric portion of $\widehat{f}_{I}$ or for the antisymmetric portion of $\widehat{f}_{\text {II }}$.

As a first example, benchmarking results as functions of $\nu^{\star}$ and $v_{E}^{\star}$ are compared in Figure 22 for the LHD standard configuration. The results for the equivalent axisymmetric tokamak are shown by the dotted curve and the predicted asymptotic value of $D_{31}^{\star}$ in the collisionless limit $[82,83]$ has been evaluated numerically for LHD and is given by the dot-dash line. This asymptotic value is well below one (as would be expected due to partial cancellation of the contributions attributable to the toroidal curvature and helical variation of $B$ ) but unlike the case of a tokamak it does not represent the upper bound on $D_{31}^{\star}$ for this configuration. Instead, depending on the magnitude of $v_{E}^{\star}$, the numerical results can attain values more than a factor of two larger and even exceed the level of the equivalent axisymmetric tokamak in a handful of cases. This "overshoot" is strongly reduced as $v_{E}^{\star}$ increases and the larger the 


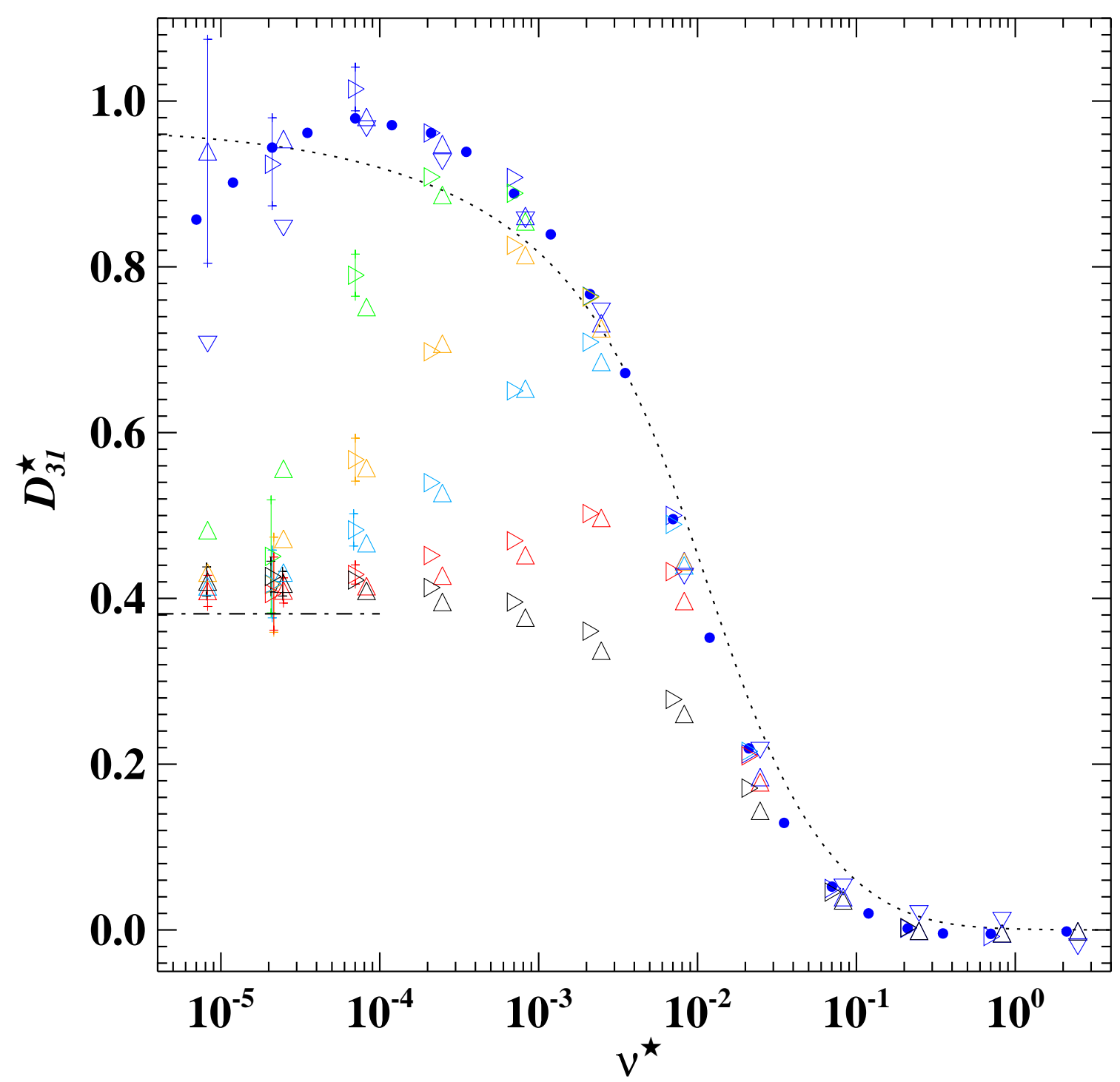

Figure 22. Normalised mono-energetic bootstrap current coefficient as a function of collisionality for $v_{E}^{\star}=3 \times 10^{-3}, 1 \times 10^{-3}, 3 \times 10^{-4}, 1 \times 10^{-4}, 3 \times 10^{-5}$ and zero for the LHD standard configuration at $\rho=0.5$. Numerical results from NEO-2 are depicted as small filled-in circles $(\bullet)$ and those from DKES as triangles $(\triangle)$ with upper and lower variational bounds indicated when these lie outside the symbol. The results from VENUS $+\delta f$ are plotted as upside-down triangles $(\nabla)$ and those from a second Monte Carlo code [15] are shown by right-pointing triangles $(\triangleright)$ with the standard error indicated when this is larger than the symbol. For comparison, results for the equivalent axisymmetric tokamak $(r=0.2793 \mathrm{~m}$, $\left.R_{0}=3.7481 \mathrm{~m}, t=0.4542, b_{1,0}=-0.07053\right)$ are shown by the dotted line for $E_{r}=0$. The collisionless asymptote [82,83] for LHD has been evaluated by numerical integration and is shown by the dot-dash line. 
normalised radial electric field becomes the more rapidly the numerical results in the $\operatorname{lmfp}$ regime approach the collisionless asymptote.

As regards the actual benchmarking for LHD, the results from the different numerical calculations of $D_{31}^{\star}$ exhibit satisfactory agreement although accuracy does suffer somewhat at the smallest values of collisionality. For DKES, the problem is the usual one at low $\nu^{\star}$ of poor convergence of the upper and lower variational bounds on the transport coefficient (at least for $v_{E}^{\star}=0$ ). As collisionality is reduced the VENUS $+\delta f$ Monte Carlo code, which employs filtering to reduce the statistical noise in its calculations of the mono-energetic bootstrap current coefficient, produces results which become increasingly dependent on the choice of filters with the numerical value for $D_{31}^{\star}$ changing by $40 \%$ for the most collisionless case as the upper limit on the perturbed distribution function was varied in the range $0.01 \leq \delta f / f_{M} \leq 0.05$. The right-pointing triangles indicate the statistical mean of results from the $\delta f$ Monte Carlo code which uses advanced weighting techniques [15] with error bars used to depict the standard error of the mean when this exceeds the size of the symbol.

Several other ICNTS configurations were found to have results for $D_{31}^{\star}\left(\nu^{\star}, v_{E}^{\star}\right)$ which, at least qualitatively, mirror those of the LHD standard configuration. CHS, the inwardshifted LHD, W7-AS and TJ-II all have mono-energetic bootstrap current coefficients which are reduced in the $\operatorname{lmfp}$ regime as the magnitude of the radial electric field is increased, until finally converging to the value of the collisionless asymptote for the configuration. Although this asymptote always satisfies $D_{31}^{\star}<1$, the maximum overshoot generally exceeds unity (reaching as high as $D_{31}^{\star} \approx 3$ for TJ-II) and occurs for small values of $v_{E}^{\star}$ at experimentally relevant collisionalities. This becomes of some practical importance when the "ion-root" solution for $E_{r}$ emerges from the ambipolarity constraint on the radial particle fluxes [24], the value of which may be often estimated by solving $\left\langle\Gamma^{i} \cdot \nabla r\right\rangle=0$, since for comparable ion and electron temperatures the radial electron particle flux is smaller than its ion counterpart by the squareroot of their mass ratio, $\left(\mathrm{m}^{e} / \mathrm{m}^{i}\right)^{1 / 2}$, if ambipolarity is ignored. Expressing the thermodynamic forces $A_{1}$ and $A_{2}$ explicitly, assuming $A_{3}$ to be negligible and setting $q^{i}=Z^{i} e$, where $Z$ is the charge state and $e$ the elementary charge, the radial electric field is then found to satisfy

$$
\frac{Z^{i} e E_{r}}{T^{i}}=\frac{1}{n^{i}} \frac{\mathrm{d} n^{i}}{\mathrm{~d} r}+\left(\frac{L_{12}^{i}}{L_{11}^{i}}-\frac{3}{2}\right) \frac{1}{T^{i}} \frac{\mathrm{d} T^{i}}{\mathrm{~d} r} .
$$

Substituting this into the expressions for the ion and electron parallel current densities yields

$$
\begin{gathered}
\frac{\left\langle\mathbf{J}^{i} \cdot \mathbf{B}\right\rangle}{e B_{0}}=-n^{i} Z^{i} L_{31}^{i}\left(\frac{L_{32}^{i}}{L_{31}^{i}}-\frac{L_{12}^{i}}{L_{11}^{i}}\right) \frac{1}{T^{i}} \frac{\mathrm{d} T^{i}}{\mathrm{~d} r} \\
\frac{\left\langle\mathbf{J}^{e} \cdot \mathbf{B}\right\rangle}{e B_{0}}=n^{e} L_{31}^{e}\left\{\frac{1}{n^{e}} \frac{\mathrm{d} n^{e}}{\mathrm{~d} r}+\left(\frac{L_{32}^{e}}{L_{31}^{e}}-\frac{3}{2}\right) \frac{1}{T^{e}} \frac{\mathrm{d} T^{e}}{\mathrm{~d} r}+\frac{1}{Z^{i}} \frac{T^{i}}{T^{e}}\left(\frac{1}{n^{i}} \frac{\mathrm{d} n^{i}}{\mathrm{~d} r}+\left(\frac{L_{12}^{i}}{L_{11}^{i}}-\frac{3}{2}\right) \frac{1}{T^{i}} \frac{\mathrm{d} T^{i}}{\mathrm{~d} r}\right)\right\} .
\end{gathered}
$$

With regard to $D_{11}$ the ions will be found predominantly in the $\sqrt{\nu}$ or $\nu$ regimes, where $L_{12}^{\alpha} / L_{11}^{\alpha}-3 / 2$ varies from $5 / 4$ to $1 / 2$, respectively [84]. The thermal transport coefficients 
obtained by convolutions of $D_{31}$ may be expected to adhere closely to the relationship valid in the collisionless limit $L_{32}^{\alpha} / L_{31}^{\alpha}-3 / 2=1$. Thus, the ion-root solution for $E_{r}$ is seen to "transfer" current from the ion to the electron channel to such an extent that the bootstrap current in the stellarator $\operatorname{lmfp}$ regime is carried chiefly by electrons for which small values of $v_{E}^{\star}$ are relevant for determining the crucial $L_{31}^{e}$ coefficient. More accurate calculations of the ion and electron bootstrap current densities - in which the ambipolarity condition is solved exactly and the flows are corrected to satisfy parallel momentum conservation - will deviate to some extent from these analytic expressions but the electron channel remains dominant when the ion-root solution for $E_{r}$ is realised [21,85].

For magnetic configurations which are approximately quasi-symmetric it is perhaps plausible to expect results for $D_{31}^{\star}$ which reflect the corresponding spatial symmetry and have little or no dependence on the radial electric field. What actually occurs is considerably more intriguing, however, as the benchmarking results for NCSX (Figure 23) and HSX (Figure 24) demonstrate. In the case of NCSX there is indeed relatively little dependence of the numerical results on $v_{E}^{\star}$ but the calculated values of $D_{31}^{\star}$ agree with those of the equivalent axisymmetric tokamak only when $\nu^{\star}>5 \times 10^{-3}$ and otherwise lie below this reference curve, the departure therefrom increasing as the collisionality is reduced. It is also notable that the numerical results show no clear tendency to converge to a constant value at the lowest $\nu^{\star}$ considered here, having already dropped below the predicted collisionless asymptote. This behaviour is confirmed by each of the computational methods and may be considered certain due to the relatively small numerical/statistical errors of the NCSX calculations.

For HSX, the most dramatic departure of the numerical results from the reference curve for the equivalent helical symmetry occurs at low collisionality for $v_{E}^{\star}=0$ and is verified by Monte Carlo, NEO-2 and DKES calculations. By performing additional DKES computations with greatly simplified magnetic field spectra it was found that such behaviour appears when the strict helical symmetry of a single $b_{1,1}$ harmonic is perturbed by an additional $b_{m, 1}$ term with $m>1$; in this particular example when $b_{1,1}=-0.07039$ is augmented by $b_{2,1}=-0.00268$. Although the importance of such small magnetic field harmonics to the $D_{31}^{\star}$ results is rapidly suppressed by introducing modest values of $v_{E}^{\star}$ into the calculation this will often be of little relevance when determining the electron bootstrap current. Given the size and plasma parameters of HSX there is little to fear from larger negative values of the bootstrap current but for a hightemperature ion-root discharge in a larger version of this device the resulting reduction of the rotational transform might be a cause for concern, warranting further investigations in which VMEC equilibrium calculations account for the bootstrap current density profiles in a selfconsistent manner.

Strict poloidal symmetry produces zero bootstrap current and although the corresponding quasi-symmetry cannot exist, it is nonetheless possible for a stellarator which has dominant $b_{0, n}$ harmonics in its Boozer representation of $B$ to achieve extremely small values of $D_{31}^{\star}$ at 


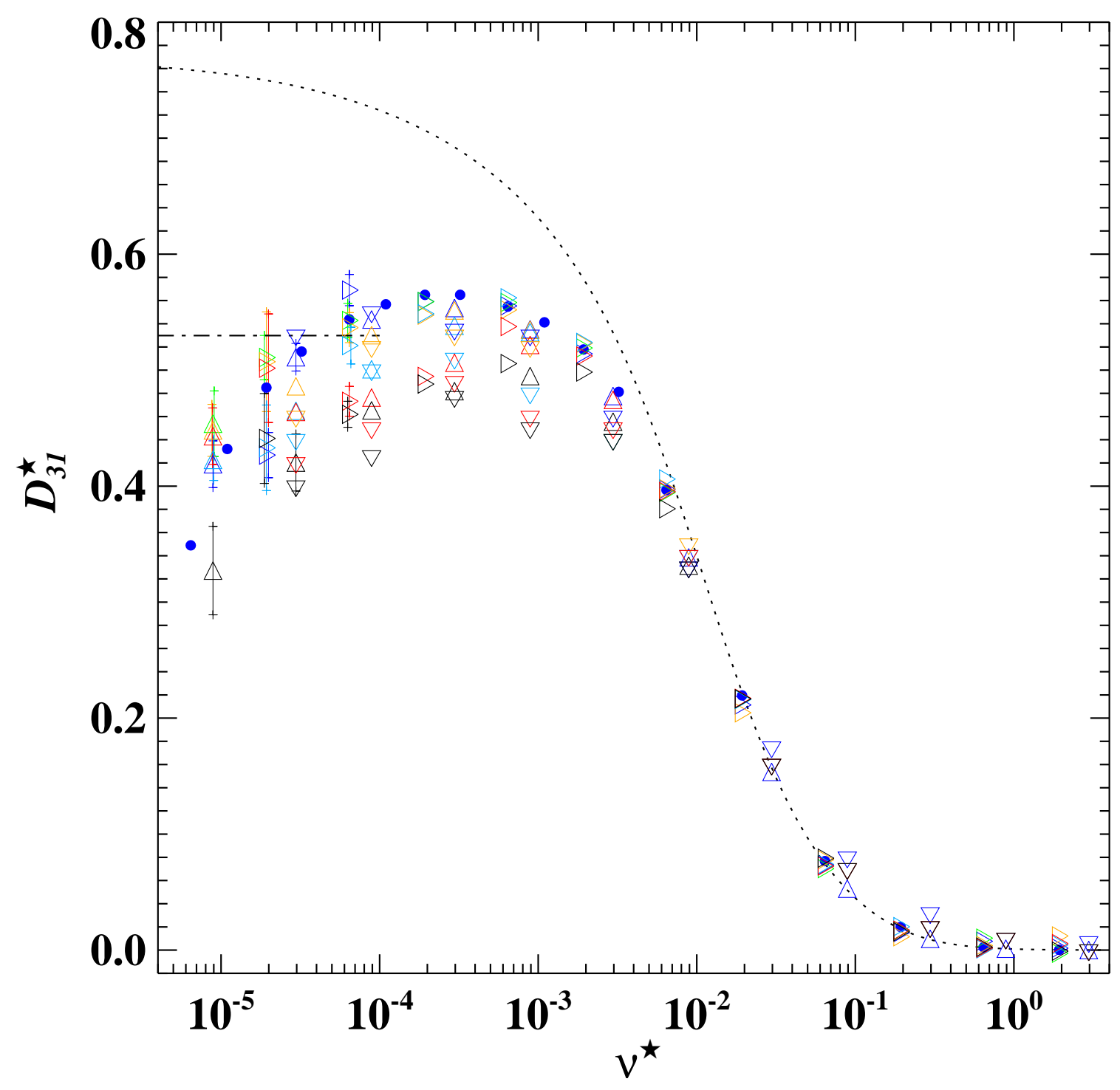

Figure 23. Normalised mono-energetic bootstrap current coefficient as a function of collisionality for $v_{E}^{\star}=3 \times 10^{-3}, 1 \times 10^{-3}, 3 \times 10^{-4}, 1 \times 10^{-4}, 3 \times 10^{-5}$ and zero for NCSX at $\rho=0.5$. Numerical results from NEO-2 are depicted as small filled-in circles $(\bullet)$ and those from DKES as triangles $(\triangle)$ with upper and lower variational bounds indicated when these lie outside the symbol. The results from VENUS $+\delta f$ are plotted as upside-down triangles $(\nabla)$ and those from a second Monte Carlo code [15] are shown by right-pointing triangles $(\triangleright)$ with the standard error indicated when this is larger than the symbol. For comparison, results for the equivalent axisymmetric tokamak $\left(r=0.1615 \mathrm{~m}, R_{0}=1.4654 \mathrm{~m}\right.$, $\left.t=0.4942, b_{1,0}=-0.06848\right)$ are shown by the dotted line for $E_{r}=0$. The collisionless asymptote $[82,83]$ for NCSX has been evaluated by numerical integration and is shown by the dot-dash line. 


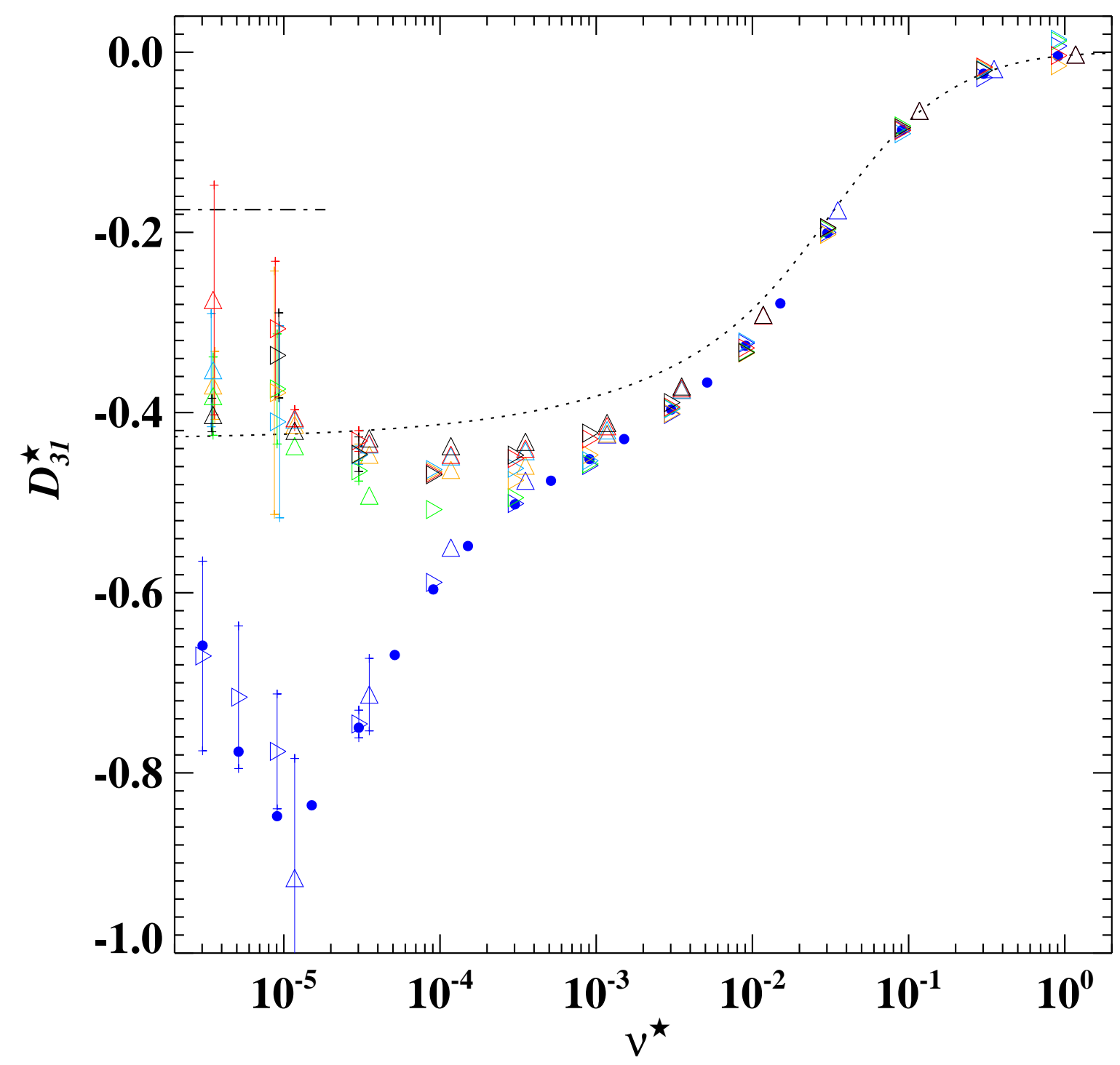

Figure 24. Normalised mono-energetic bootstrap current coefficient as a function of collisionality for $v_{E}^{\star}=3 \times 10^{-3}, 1 \times 10^{-3}, 3 \times 10^{-4}, 1 \times 10^{-4}, 3 \times 10^{-5}$ and zero for HSX at $\rho=0.5$. Numerical results from NEO-2 are depicted as small filled-in circles $(\bullet)$, those from DKES as triangles $(\triangle)$ with upper and lower variational bounds indicated when these lie outside the symbol and results from a Monte Carlo codes [15] are plotted as right-pointing triangles $(\triangleright)$ with the standard error indicated when this is larger than the symbol. For comparison, results for the equivalent helical symmetry $(r=0.0598 \mathrm{~m}$, $\left.R_{0}=1.2375 \mathrm{~m}, t=1.0537, N=4, b_{1,1}=-0.07039\right)$ are shown by the dotted line for $E_{r}=0$. The collisionless asymptote $[82,83]$ for HSX has been evaluated by numerical integration and is shown by the dot-dash line. 


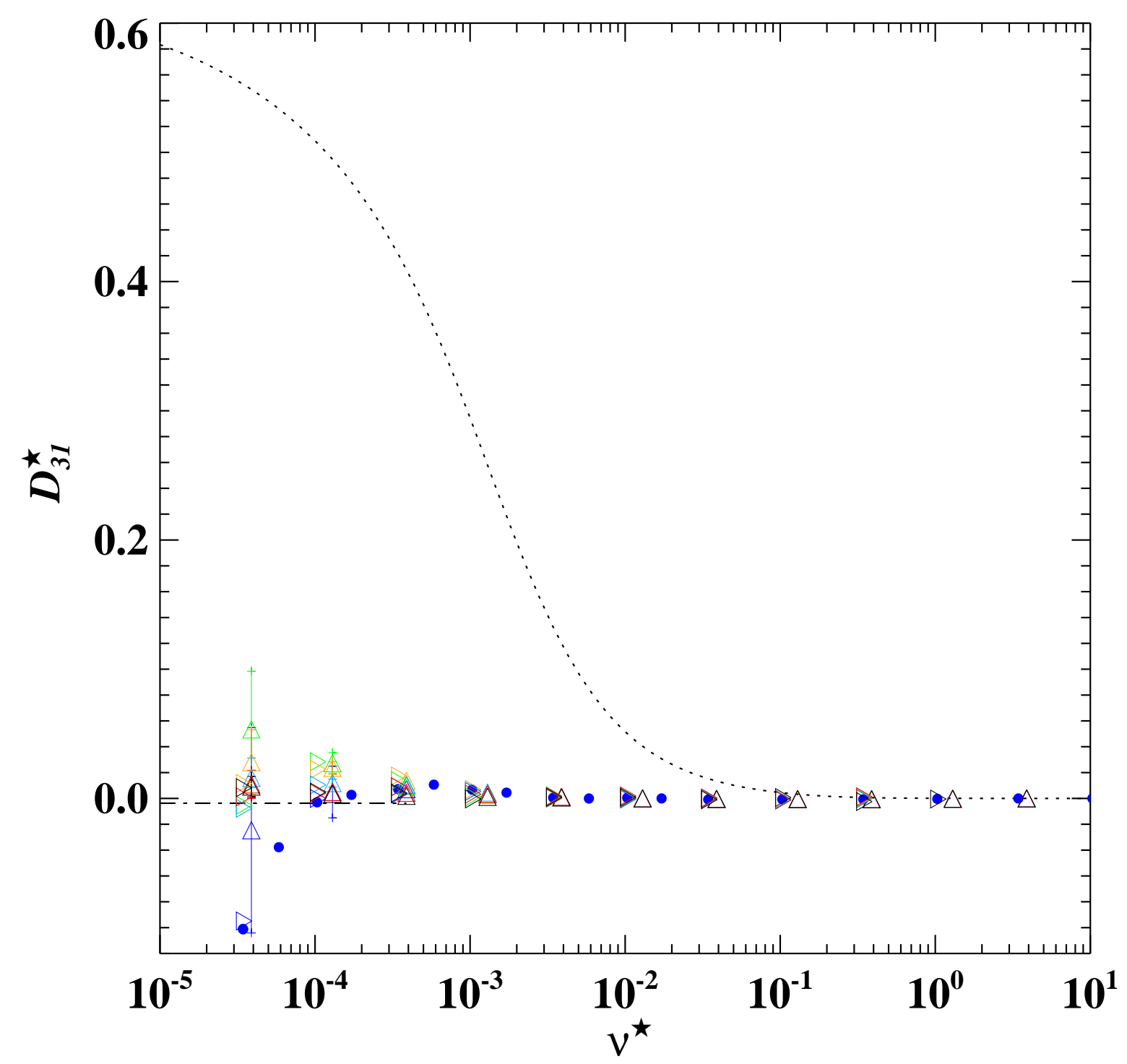

Figure 25. Normalised mono-energetic bootstrap current coefficient as a function of collisionality for $v_{E}^{\star}=3 \times 10^{-3}, 1 \times 10^{-3}, 3 \times 10^{-4}, 1 \times 10^{-4}, 3 \times 10^{-5}$ and zero for QIPC at $\rho=0.5$. Numerical results from NEO-2 are depicted as small filled-in circles $(\bullet)$, those from DKES as triangles $(\triangle)$ with upper and lower variational bounds indicated when these lie outside the symbol and results from a Monte Carlo codes are plotted as right-pointing triangles $(\triangleright)[15]$. For comparison, results for the equivalent axisymmetric tokamak ( $\left.r=0.5135 \mathrm{~m}, R_{0}=11.846 \mathrm{~m}, t=0.9137, b_{1,0}=-0.01689\right)$ are shown by the dotted line for $E_{r}=0$. The collisionless asymptote [82,83] for QIPC has been evaluated by numerical integration and is shown by the dot-dash line. 


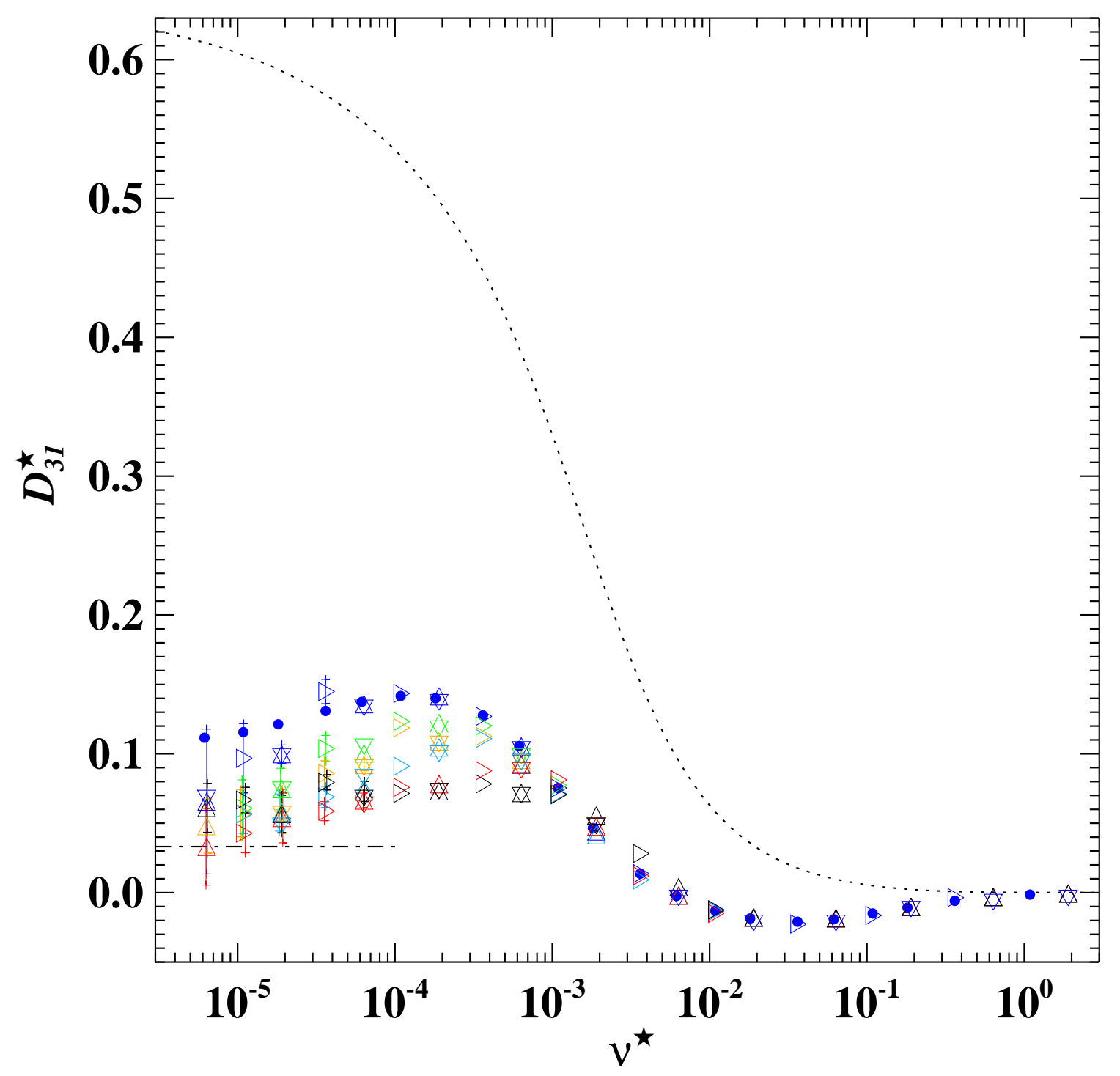

Figure 26. Normalised mono-energetic bootstrap current coefficient as a function of collisionality for $v_{E}^{\star}=3 \times 10^{-3}, 1 \times 10^{-3}, 3 \times 10^{-4}, 1 \times 10^{-4}, 3 \times 10^{-5}$ and zero for the W7-X standard configuration at $\rho=0.5$. Numerical results from NEO-2 are depicted as small filled-in circles $(\bullet)$ and those from DKES as triangles $(\triangle)$ with upper and lower variational bounds indicated when these lie outside the symbol. The results from VENUS $+\delta f$ are plotted as upside-down triangles $(\nabla)$ and those from a second Monte Carlo code [15] are shown by right-pointing triangles $(\triangleright)$ with the standard error indicated when this is larger than the symbol. For comparison, results for the equivalent axisymmetric tokamak $(r=0.2555 \mathrm{~m}$, $\left.R_{0}=5.5267 \mathrm{~m}, t=0.870, b_{1,0}=-0.01902\right)$ are shown by the dotted line for $E_{r}=0$. The collisionless asymptote $[82,83]$ for $\mathrm{W} 7-\mathrm{X}$ has been evaluated by numerical integration and is shown by the dot-dash line. 
experimentally relevant collisionalities. The best ICNTS example of such a configuration is QIPC, for which the benchmarking results, depicted in Figure 25, only depart appreciably from zero for $v_{E}^{\star}=0$ at the lowest value of $\nu^{\star}$ considered here. As in the case of HSX, it is suspected that the $b_{m, n}$ with poloidal index one larger than that of the dominant magnetic field harmonic ( $b_{1,1}=0.04338$ in addition to $b_{0,1}=-0.22879$ in this example) is largely responsible for this departure but an attempt to verify this numerically has not yet been undertaken. Considerable effort was made in the design of QIPC to align not only the local minima of $B$ along a field line but to achieve the same for the maxima as well (see the bottom frame of Figure 8). Values of the normalised mono-energetic bootstrap current coefficient are significantly larger for a quasiisodynamic device without the latter property; for example, QPS was found to have values of $D_{31}^{\star}$ as large as 0.25 in the $\operatorname{lmfp}$ regime for the flux surface $\rho=0.5$.

An alternative strategy for reduction of the bootstrap current in stellarators is to more fully exploit the cancellation of "toroidal" and "helical" contributions to $D_{31}^{\star}$, as was done in the optimisation of W7-X by choosing a magnetic field structure with appropriate magnitudes of the two harmonics $b_{1,0}$ and $b_{1,1}$ [70]. The benchmarking results for the W7-X standard configuration, plotted in Figure 26, demonstrate the viability of such an optimisation strategy but also illustrate its limitations; numerical values of $D_{31}^{\star}$ never exceed 0.15 but nonetheless exhibit the same overshoot of the collisionless asymptote for small values of $v_{E}^{\star}$ displayed by the LHD results. For the expected plasma parameters of W7-X this would imply a bootstrap current large enough to cause experimentally relevant alterations to the magnetic topology of the island divertor in the standard configuration [86]. Similar conclusions may be drawn for the W7-X low-mirror configuration for which the normalised mono-energetic bootstrap current coefficient reaches values as high as 0.3 (for $v_{E}^{\star}=0$ ), which is an order of magnitude larger than the collisionless asymptote. Such overshoot is steadily reduced, however, by increasing the magnitude of the mirror term in the $B$ spectrum of W7-X as the ICNTS benchmarking activity has been able to confirm for the high-mirror configuration. Indeed, the generally observed behaviour of the $D_{31}^{\star}$ results with $v_{E}^{\star}$ at low collisionality is reversed in this case with the smallest values (satisfying $D_{31}^{\star} \leq 0.01$ ) occurring for zero electric field over the relevant range of collisionalities. Consequently, the bootstrap current in the W7-X high-mirror configuration is not expected to exceed a negligible level on the order of a few kA even when accounting for the small reduction in $b_{0,1}$ which occurs for finite- $\beta$ equilibria.

Data points of two calculations for W7-X performed with the VENUS $+\delta f$ Monte Carlo code do not appear in Figure 26. These were carried out for $E_{r}=0$ with the results $D_{31}^{\star}=0.047$ at $\nu^{\star}=1.9 \times 10^{-6}$ and $D_{31}^{\star}=0.040$ at $\nu^{\star}=6.4 \times 10^{-7}$. These values are consistent with a gradual convergence to the predicted collisionless asymptote but also demonstrate how extreme the collisionality must become in certain cases before this asymptote is of any practical relevance. As a consequence, determining the value of $D_{31}^{\star}$ for a configuration in the collisionless limit — which would otherwise be attractive as a figure of merit since it depends 
only on the structure of $B$ - will often provide a rather poor measure for the magnitude of the bootstrap current to be expected under realistic experimental conditions.

\section{Comments and Conclusions}

The principal task of the ICNTS benchmarking activity was to compare the accuracy and applicability of various numerical tools used within the stellarator community for determining mono-energetic transport coefficients in realistic magnetic fields; the successful completion of this task is demonstrated by the representative sample of results presented in the previous section of this paper. Beyond this formal benchmarking, however, the calculations also provide a wealth of additional information concerning neoclassical transport in stellarators and a few observations are sufficiently noteworthy to be included in the following summary of results.

- Of the numerical tools used in the ICNTS benchmarking, the $\delta f$ Monte Carlo codes have the widest range of applicability as they are able to determine the mono-energetic transport coefficients in arbitrarily complex magnetic fields for given values of the normalised $\mathbf{E}_{r} \times \mathbf{B}$ drift velocity, $\left(v_{E}^{\star}=E_{r} /\left(v B_{0}\right)\right)$ at values of the mono-energetic collisionality $\left(\nu^{\star}=R_{0} \nu /(t v)\right)$ which are limited only by the available computational resources; as an extreme example, the calculation of the mono-energetic bootstrap current coefficient $\left(D_{31}\right)$ for the W7-X standard configuration at $\nu^{\star}=6.4 \times 10^{-7}$ by VENUS $+\delta f$ consumed the equivalent of 155 days of process time on $2.66 \mathrm{GHz}$ processors (more typical is the 40 hours of process time on $3.06 \mathrm{GHz}$ processors required for LHD at $\nu^{\star}=7.0 \times 10^{-5}$ by the $\delta f$ approach employing advanced weighting techniques). Similar claims can be made for the orbit-following Monte Carlo codes with two notable caveats: determination of the mono-energetic radial transport coefficient $\left(D_{11}\right)$ becomes impossible with this approach for $\nu^{\star}$ values below the threshold at which non-local transport appears in the simulations and calculations of $D_{31}$ using such codes were not undertaken here. In the former case it is possible to view this failure in a positive light as it is a clear indication that the local ansatz underlying neoclassical theory becomes unrealistic for particles with such small values of $\nu^{\star}$; in the latter case the lack of results makes any verdict impossible. Use of the Drift Kinetic Equation Solver (DKES) is also attractive as it allows simultaneous determination of the three mono-energetic transport coefficients for specified values of $\nu^{\star}$ and $v_{E}^{\star}$ in magnetic fields of arbitrary complexity. Convergence of the upper and lower variational bounds on the DKES results worsens as the collisionality decreases, however, becoming unacceptably poor for (rather) small values of $\nu^{\star}$; DKES test functions have been circumscribed here so as to require at most 30 hours of process time for a single calculation performed with $2.60 \mathrm{GHz}$ processors as the convergence of the bounds is not improved significantly even by a ten-fold expenditure of computational resources. Solution of the kinetic equation using the field-line following approach of the NEO codes is far more 
efficient numerically regardless of the complexity of $B$, requiring at most five hours of process time on $2.60 \mathrm{GHz}$ processors to determine the three mono-energetic transport coefficients at the smallest values of $\nu^{\star}$ considered and determining the effective helical ripple $\left(\epsilon_{e f f}\right.$, which characterises $D_{11}$ in the $1 / \nu$ regime) in a fraction of a second. Field-line following assumes $v_{E}^{\star}=0$, however, and although this is of no consequence in determining values of the parallel transport coefficient ( $D_{33}$ being independent of $E_{r}$ ), practical use of the results for $D_{11}$ and $D_{31}$ is thus confined to electrons. Use of the General Solution of the Ripple-Averaged Kinetic Equation (GSRAKE) was limited in the ICNTS benchmarking to CHS, LHD and W7-X, i.e. to those magnetic configurations with field structures simple enough to allow accurate performance of the averaging. When appropriate, however, GSRAKE offers the advantage of very rapid calculation of $D_{11}$ in the presence of a radial electric field, consuming only a few seconds of process time even for extremely small values of collisionality.

- Neoclassical fluxes/flows for a stellarator plasma may be determined very efficiently assuming that a precalculated database of the three mono-energetic transport coefficients already exists for the magnetic configuration of interest at experimentally relevant values of $\nu^{\star}$ and $v_{E}^{\star}$. The creation of such a database consumes considerable computational resources, however, and it is therefore desirable to keep the number of expensive calculations to a minimum. Thus, at low collisionality, having results from more than one Monte Carlo code or from both DKES and Monte Carlo computations will be the exception rather than the rule. The ICNTS benchmarking results allow one to view this situation with equanimity as the isolated disparities observed in the numerical computations of the $D_{i j}$ (in the worst case, differences as large as a factor of two for individual calculations of $D_{11}$ ) have at most a modest effect on the weighted energy convolutions with the local Maxwellian which must be performed to determine the $L_{i j}$ (the elements of the thermal transport matrix).

- The "density" of entries in the database should be at least two per decade throughout the relevant ranges of $\nu^{\star}$ and $v_{E}^{\star}$ values (this number was commonly used during the ICNTS benchmarking) for at least seven flux surfaces which adequately represent the variation of the results in the radial direction. This implies performing a minimum of around 500 separate computations for a single magnetic configuration although this number increases rapidly when greater radial resolution is required. Interpolation within the dataset is done using standard algorithms or with the aid of a neural network [12], which is especially attractive when the dataset is extended by an additional dimension to also describe the $\beta$ dependence of the results [87] arising due to the influence of the plasma pressure on the structure of $B$.

- Extrapolation outside the dataset is a different matter. In the analysis of current experimental data this is required most often for high-temperature, low-density discharges for which a nonnegligible portion of the local Maxwellian finds itself at collisionalities less than the smallest value of $\nu^{\star}$ in the dataset. There is no entirely satisfactory solution to this problem but the benchmarking results offer ways to proceed in some specific cases. For example, in stellarators 
at these collisionalities momentum conservation yields a totally negligible correction to the neoclassical radial particle and energy fluxes [21] so that knowledge of $D_{11}^{\star}$ alone is sufficient for their determination. Consequently, the $D_{11}^{\star}$ database of Monte Carlo results used to analyse LHD discharges has been extended to lower collisionalities by performing additional calculations with GSRAKE [88]. The results of magnetic configurations for which GSRAKE is not applicable can also be "extended" using the observation that the scaling of $D_{11}$ with collision frequency and radial electric field behaves consistently with the analytic results derived for classical stellarators [6]. Extending $D_{33}^{\star}$ results is also straightforward, either with additional NEO-2 calculations or using an asymptotic extrapolation to the fraction of circulating particles. The greatest difficulties arise for the mono-energetic bootstrap current coefficient which exhibits a dependence on the radial electric field which is not theoretically understood and for which the low-collisionality results do not always conform with the asymptotic predictions. Thus, extension of the $D_{31}^{\star}$ results under physics considerations is not possible and instead one resorts to ad hoc models such as setting $D_{31}^{\star}\left(\nu^{\star}, v_{E}^{\star}\right)=D_{31}^{\star}\left(\nu_{\text {min }}^{\star}, v_{E}^{\star}\right)$ for $\nu^{\star}<\nu_{\text {min }}^{\star}$, where $\nu_{\text {min }}^{\star}$ is the smallest value of collisionality for which numerical results are available [89]. In this regard, the benchmarking results document the shortcomings of the existing theoretical descriptions of the bootstrap current in stellarators and indicate topics for future investigation.

- The energy confinement time of existing stellarator experiments increases roughly with the square root of the line-averaged density $[49,90]$ and operation is possible well above the equivalent Greenwald density limit observed in tokamaks [91]. In stellarator reactor studies it is thus common to consider high-density ( $n^{e}(0)$ between 2 and $4 \times 10^{20} \mathrm{~m}^{-3}$ ), low-temperature $(T(0)$ between 12 and $18 \mathrm{keV}$ ) operation as particularly attractive [92,93]. Predictive transport simulations for such plasma parameters are straightforward as far as neoclassical theory is concerned as the resultant range of collisionalities never encompasses values of $\nu^{\star}$ which are inaccessible to any of the computational tools. From the viewpoint of confinement, operation at higher collisionality is favourable as it leads to a reduction of electron $1 / \nu$ losses and a corresponding drop in ion transport is brought about by the ambipolar radial electric field. For stellarators with sufficiently small effective helical ripple, $\epsilon_{e f f}$, the neoclassical confinement is then sufficiently good to allow ignition in a device of reactor dimensions as has been shown for a scaled-up version of W7-X with $R_{0}=22 \mathrm{~m}, a=1.8 \mathrm{~m}$ and $B_{0}=5 \mathrm{~T}$ for $n^{e}(0) \approx 3.9 \times 10^{20} \mathrm{~m}^{-3}$ and $T(0) \approx 13 \mathrm{keV}$ [92]. Evaluating the reactor prospects of all ICNTS configurations with regards to neoclassical confinement is beyond the scope of the current paper (a superficial comparison with the $\mathrm{W} 7-\mathrm{X}$ reactor is possible by noting that the electron energy diffusivity scales as $\chi^{e} \propto \epsilon_{\text {eff }}^{3 / 2}\left(T^{e}\right)^{7 / 2} /\left(n^{e} R_{0}^{2} B_{0}^{2}\right)$ in the $1 / \nu$ regime) although the benchmarking results for a given configuration can be scaled to arbitrary values of $B_{0}$ and $R_{0}$ (at fixed aspect ratio) making it possible to carry out such an evaluation in future work.

With benchmarking having reached its conclusion, the emphasis of the ICNTS has shifted to development and testing of the theoretical and numerical tools required for practical application 
of the results. This includes benchmarking of the various momentum-correction techniques [19-21] used to restore conservation of parallel momentum to the calculation of the neoclassical fluxes/flows and a comparison of the "neoclassical packages" used by different 1-D transport codes. Reports on these activities will be provided in future publications.

\section{Acknowledgements}

The authors would like to thank:

- S. Okamura and M. Yokoyama for providing the magnetic configuration of CHS,

- J. N. Talmadge for the magnetic configuration of HSX,

- M. I. Mikhailov and J. Nührenberg for the magnetic configuration of QIPC,

- W. A. Cooper for performing the TERPSICHORE calculations of magnetic coordinates needed as input by the VENUS $+\delta f$ code,

- J. L. Velasco for providing the Monte Carlo results for $D_{11}^{\star}$ from [15] for TJ-II at very-low $\nu^{\star}$,

- J. Geiger for numerous VMEC calculations and

- J. Kißlinger for the subroutine which calculates the asymptotic value of the mono-energetic bootstrap current.

Portions of the numerical results were obtained using:

- the NEC-SX5 platform at the CSCS (Manno),

- the Pleiades cluster (EPFL, Switzerland) and

- the facilities and expertise of the computational centre at CIEMAT (Madrid, Spain).

This work was supported in part by:

- the Austrian Science Foundation, FWF, under contract number P16797-N08,

- the Russian-German Agreement WTZ-RUS-01-581,

- the Russian Federal Programme for the Support of Leading Scientific Schools, Grant N 2024.2003.2,

- the Russian Fund for Basic Research, Grant N 03-02-16768,

- the Department of Atomic Science and Technology, RosAtom, Russian Federation,

- the Fonds National Suisse pour la Recherche Scientifique,

- the United States Department of Energy under contract DE-AC02-09CH11466,

- the United States Department of Energy under contract DE-AC05-00OR22725 with UT-

Battelle, LLC,

- the Spanish project MICINN ENE 2009-12213-C03-03/FTN,

- the Associations IPP-EURATOM, ÖAW-EURATOM and EURATOM-CIEMAT (the content of this publication is the sole responsibility of its authors and does not necessarily represent the views of the European Commission or its services). 


\section{References}

[1] Baldzuhn J, Kick M, Maaßberg H and the W7-AS Team 1998 Plasma Phys. Control. Fusion 40967

[2] Dinklage A et al 2007 Nucl. Fusion 471265

[3] Hirsch M et al 2008 Plasma Phys. Control. Fusion 50053001

[4] Yokoyama M et al 2006 Fusion Sci. Technol. 50327

[5] Yokoyama M et al 2007 Nucl. Fusion 471213

[6] Galeev A A and Sagdeev R Z 1979 Theory of Neoclassical Diffusion Reviews of Plasma Physics vol 7 (New York: Consultants Bureau, editor M A Leontovich) p. 257

[7] Kovrizhnykh L M 1984 Nucl. Fusion 24851

[8] Nemov V V, Kasilov S V, Kernbichler W and Heyn M F 1999 Phys. Plasmas 64622

[9] Kernbichler W, Kasilov S V, Leitold G O, Nemov V V and Allmaier K 2008 Plasma Fusion Res. 3 S1061

[10] Beidler C D, Hitchon W N G and Shohet J L 1987 J. Comput. Phys. 72220

[11] Wakasa A, Murakami S, Maaßberg H, Beidler C D, Nakajima N, Watanabe K, Yamada H, Okamoto M, Oikawa S and Itagaki M 2001 J. Plasma Fusion Res. SERIES 4408

[12] Tribaldos V 2001 Phys. Plasmas 81229

[13] Schmidt M 2002 Proc. 29th EPS Conf. Plasma Phys. Control. Fusion (Montreux, 2002) vol 26B http://epsppd.epfl.ch/Montreux/pdf/P4_112.pdf

[14] Isaev M Yu, Brunner S, Cooper W A, Tran T M, Bergmann A, Beidler C D, Geiger J, Maaßberg H, Nührenberg J and Schmidt M 2006 Fusion Sci. Technol. 50440

[15] Allmaier K, Kasilov S V, Kernbichler W and Leitold G O 2008 Phys. Plasmas 15072512

[16] Hirshman S P, Shaing K C, van Rij W I, Beasley C O and Crume E C 1986 Phys. Fluids 292951

[17] van Rij W I and Hirshman S P 1989 Phys. Fluids B 1563

[18] Beidler C D and D'haeseleer W D 1995 Plasma Phys. Control. Fusion 37463

[19] Taguchi M 1992 Phys. Fluids B 43638 
[20] Sugama H and Nishimura S 2002 Phys. Plasmas 94637

[21] Maaßberg H, Beidler C D and Turkin Y 2009 Phys. Plasmas 16072504

[22] Hinton F L and Hazeltine R D 1976 Rev. Mod. Phys. 48239

[23] Mynick H E 1983 Phys. Fluids 262609

[24] Mynick H E and Hitchon W N G 1983 Nucl. Fusion 231053

[25] Romé M, Erckmann V, Gasparino U and Karulin N 1998 Plasma Phys. Control. Fusion 40511

[26] Boozer A H and Gardner H J 1990 Phys. Fluids B 22408

[27] Potok R E, Politzer P A and Lidsky L M 1980 Phys. Rev. Lett. 451328

[28] Boozer A H and Kuo-Petravic G 1981 Phys. Fluids 24851

[29] Fowler R H, Rome J A and Lyon J F 1985 Phys. Fluids 28338

[30] Boozer A H 1980 Phys. Fluids 23904

[31] Wobig H 1982 Z. Naturforsch. 37a 906

[32] Tribaldos V and Guasp J 2005 Plasma Phys. Control. Fusion 47545

[33] Maaßberg H, Lotz W and Nührenberg J 1993 Phys. Fluids B 53728

[34] Matsuyama A and Hanatani K 2010 Phys. Plasmas 17032501

[35] Belli E A and Candy J 2008 Plasma Phys. Control. Fusion 50095010

[36] Spong D A et al 2001 Nucl. Fusion 41711

[37] Shaing K C and Hokin S A 1983 Phys. Fluids 262136

[38] Beidler C D and Maaßberg H 2001 Plasma Phys. Control. Fusion 431131

[39] Spitzer L 1951 Project Matterhorn Report PM-S-1 (23 July, 1951)

[40] Spitzer L 1958 Phys. Fluids 1253

[41] Wobig H and Rehker S 1972 Proc. 7th Symposium Fusion Tech. (Grenoble, 1972) (Luxembourg: CID of the CEC) p. 345

[42] Hall L S and McNamara B 1975 Phys. Fluids 18552 
[43] Cary J R and Shasharina S G 1997 Phys. Plasmas 43323

[44] Nishimura K et al 1990 Fusion Technol. 1786

[45] Hirshman S P, van Rij W I and Merkel P 1986 Comput. Phys. Commun. 43143

[46] Iiyoshi A, Fujiwara M, Motojima O, Ohyabu N and Yamazaki K 1990 Fusion Technol. 17 169

[47] Mynick H E, Chu T K and Boozer A H 1982 Phys. Rev. Lett. 48322

[48] Kaneko O et al 2002 Phys. Plasmas 92020

[49] Yamada H et al 2005 Nucl. Fusion 451684

[50] Okamura S et al 1999 Nucl. Fusion 391337

[51] Mynick H E 1985 Phys. Fluids 281139

[52] Brossmann U et al in Plasma Physics and Controlled Nuclear Fusion Research 1982 (Proc. 9th Int. Conf. Baltimore, 1982), Vol. III, p. 141, IAEA, Vienna (1983)

[53] Hender T C et al 1988 Fusion Technol. 13521

[54] Alejaldre C et al 1990 Fusion Technol. 17131

[55] Nührenberg J and Zille R 1988 Phys. Lett. A 129113

[56] Nührenberg J, Lotz W and Gori S 1994 Quasi-Axisymmetric Tokamaks Theory of Fusion Plasmas (Varenna) (Bologna: Editrice Compositori) p. 3

[57] Bernardin M P, Moses R W and Tataronis J A 1986 Phys. Fluids 292605

[58] Gori S, Lotz W and Nührenberg J 1996 Quasi-Isodynamic Stellararors Theory of Fusion Plasmas (Varenna) (Bologna: Editrice Compositori) p. 335

[59] Palumbo D 1968 Nuovo Cimento B X53 507

[60] Garren D and Boozer A H 1991 Phys. Fluids B 32822

[61] Anderson F S B, Almagri A F, Anderson D T, Matthews P G, Talmadge J N and Shohet J L 1995 Fusion Technol. 27273

[62] Canik J M, Anderson D T, Anderson F S B, Likin K M, Talmadge J N and Zhai K 2007 Phys. Rev. Lett. 98085002 
[63] Talmadge J N, Anderson F S B, Anderson D T, Deng C, Guttenfelder W, Likin K M, Lore J, Schmitt J C and Zhai K 2008 Plasma Fusion Res. 3 S1002

[64] Zarnstorff M C et al 2001 Plasma Phys. Control. Fusion 43 A237

[65] Pomphrey N et al 2007 Fusion Sci. Technol. 51181

[66] Koenig R et al 2002 Plasma Phys. Control. Fusion 442365

[67] Helander P and Nührenberg J 2009 Plasma Phys. Control. Fusion 51055004

[68] Spong D A, Hirshman S P, Lyon J F, Berry L A and Strickler D J 2005 Nucl. Fusion 45 918

[69] Subbotin A A et al 2006 Nucl. Fusion 46921

[70] Beidler C D et al 1990 Fusion Technol. 17148

[71] Grieger G, Beidler C D, Harmeyer E, Lotz W, Kisslinger J, Merkel P, Nührenberg J, Rau F, Strumberger E and Wobig H 1992 Fusion Technol. 211767

[72] Nührenberg J and Zille R 1986 Phys. Lett. 114A 129

[73] Lotz W, Nührenberg J and Schwab C in Plasma Physics and Controlled Nuclear Fusion Research 1990 (Proc. 13th Int. Conf. Washington DC, 1990), Vol. 2, p. 603, IAEA, Vienna (1991)

[74] Lotz W, Merkel P, Nührenberg J and Strumberger E 1992 Plasma Phys. Control. Fusion 341037

[75] Dommaschk W, Lotz W and Nührenberg J 1984 Nucl. Fusion 24794

[76] Beidler C D and Hitchon W N G 1994 Plasma Phys. Control. Fusion 36317

[77] Murakami S et al 2007 Fusion Sci. Technol. 51112

[78] Igitkhanov Y, Polunovsky E and Beidler C D 2006 Fusion Sci. Technol. 50268

[79] Rodriguez-Solano-Ribeiro E and Shaing K C 1987 Phys. Fluids 30462

[80] Boozer A H 1983 Phys. Fluids 26496

[81] Beidler C D and Maaßberg H 1996 Neoclassical Transport in Advanced Stellararors Theory of Fusion Plasmas (Varenna) (Bologna: Editrice Compositori) p. 375

[82] Shaing K C and Callen J D 1983 Phys. Fluids 263315 
[83] Nakajima N, Okamoto M, Todoroki J, Nakamura Y and Wakatani M 1989 Nucl. Fusion 29605

[84] Maaßberg H, Beidler C D and Simmet E E 1999 Plasma Phys. Control. Fusion 411135

[85] Maaßberg H and Beidler C D 2010 Phys. Plasmas 17052507

[86] Turkin Yu, Maaßberg H, Beidler C D, Geiger J and Marushchenko N B 2006 Fusion Sci. Technol. 50387

[87] Wakasa A, Murakami S, Itagaki M and Oikawa S 2007 Jpn. J. Appl. Phys. 461157

[88] Wakasa A, Murakami S, Fukuyama A, Beidler C D, Maaßberg H, Yokoyama M and Sato M 2010 Contrib. Plasma Phys. 50582

[89] Spong D A 2005 Phys. Plasmas 12056114

[90] Stroth U, Murakami M, Dory R A, Yamada H, Okamura S, Sano F and Obiki T 1996 Nucl. Fusion 361063

[91] Greenwald M 2002 Plasma Phys. Control. Fusion 44 R27

[92] Beidler C D et al in Fusion Energy 1996 (Proc. 16th Int. Conf. Montreal, 1996), Vol. 3, p. 407, IAEA, Vienna (1997)

[93] Sagara A et al 2006 Fusion Eng. Des. 812703 